Experimental Tests of a

Radiation Direction Finder

RECEIVED

DEC 121996

OSTI 
Edited by Martha Lee DeLanoy, Group CIC-1

This work was supported by the U.S. Department of Energy, Office of Research and Development of the Office of Nonproliferation and National Security.

An Affirmative Action/Equal Opportunity Employer

This report was prepared as an account of work sponsored by an agency of the United States Government. Neither The Regents of the University of Califormia, the United States Government nor any agency thereof, nor any of their employees, makes any warranty, express or implied, or assumes any legal liability or responsibility for the accuracy, completeness, or usefulness of any information, apparatus, product, or process disclosed, or represents that its use would not infringe privately owned rights. Reference herein to any specific commercial product, process, or service by trade name, trademark, manufacturer, or otherwise, does not necessarily constitute or imply its endorsement, recommendation, or favoring by The Regents of the University of California, the United States Government, or any agency thereof. The views and opinions of authors expressed herein do not necessarily state or reflect those of The Regents of the University of Californin, the United States Government, or any agency thereof. The Los Alamos National Laboratory strongly supports academic freedom and a researcher's right to publish; therefore, the Laboratory as an institution does not endorse the viewpoint of a publication or guarantee its technical correctness. 
UC-700 and UC-706 Issued: November 1996

Experimental Tests of a

Radiation Direction Finder

R. C. Byrd

P. A. Russo 


\section{DISCLAIMER}

Portions of this document may be illegible in electronic image products. Images are produced from the best available original document. 


\section{DISCLAIMER}

This report was prepared as an account of work sponsored by an agency of the United States Government. Neither the United States Government nor any agency thereof, nor any of their employees, make any warranty, express or implied, or assumes any legal liability or responsibility for the accuracy, completeness, or usefulness of any information, apparatus, product, or process disclosed, or represents that its use would not infringe privately owned rights. Reference herein to any specific commercial product, process, or service by trade name, trademark, manufacturer, or otherwise does not necessarily constitute or imply its endorsement, recommendation, or favoring by the United States Government or any agency thereof. The views and opinions of authors expressed herein do not necessarily state or reflect those of the United States Government or any agency thereof. 


\title{
EXPERIMENTAL TESTS OF A RADIATION DIRECTION FINDER
}

\author{
by
}

R. C. Byrd and P. A. Russo

\begin{abstract}
We explore the possibility of using a simple detector to determine the direction or heading toward a source of nuclear radiation, typically penetrating neutrons or gamma rays. The technique is based on the attenuation of incident radiation across a scintillator that has been segmented so that differences between the count rates provide the orthogonal components of the local radiation vector. A number of simple measurements are carried out using plutonium, californium, and gamma-ray sources at distances of 1-20 m, with results that are in good agreement with earlier Monte Carlo predictions. In addition, triangulation allows the distance to the source to be estimated by correlating the observations from two or more detectors. These initial studies suggest that directional detectors may be useful in applications such as searching for nuclear materials or monitoring the location of radiation sources in storage vaults.
\end{abstract}

\section{INTRODUCTION}

Motivation. Typically, searchers for radiation sources use a single detector to measure count rates at several locations, and they rely on differences between these rates to determine whether a source is located nearby. If the differences are large enough, they can be used to estimate a likely direction or heading toward the source. The basis for such a grid search is the nominal $1 / r^{2}$ variation in source fluence with separation distance, and in principle its success is limited mainly by the counting statistics associated with weak sources, large separation distances, or short accumulation times. In practice, a more important problem is usually the variation in local backgrounds, which makes it difficult to measure a change in the $1 / r^{2}$ signal. This report describes an alternative directional approach, which relies on the attenuation of incident radiation across a segmented detector. Any difference between the count rates for different segments immediately indicates the presence of, and the direction toward, a nearby source of radiation. Presumably, moving the detector in the indicated direction will result in higher count rates and rapid convergence on the source position. In another possible application, instead of moving the detector to locate a stationary source, one can use a stationary detector to monitor a moving source, as in a storage vault for radioactive materials. Again, the pattern of count rates across the segmented detector depends on the direction of the local radiation field, so moving any of the sources alters the field and 
changes the count rates. In these two applications, source searches and vault monitoring, segmenting a single-element detector provides additional information and greater capability with little increase in the complexity of the detector or analysis. This report describes the first substantial experimental tests of these concepts.

Previous Work. Earlier reports have provided the basis for determining the direction of a radiation field by measuring the attenuation of the radiation across a segmented, semitransparent detector. The initial evaluation ${ }^{1}$ was pursued largely through computer simulations that examined the effectiveness of electronic collimation at providing directionality for neutron detectors. To establish the validity of the concept and provide a mathematical framework for its application, two later reports ${ }^{2,3}$ reanalyzed neutron measurements originally made for other purposes. Another study ${ }^{4}$ adapted the technique from an array of plastic scintillators to a polyethylene-moderated neutron detector with multiple ${ }^{3} \mathrm{He}$ tubes. After accounting for the energy-dependent ${ }^{3} \mathrm{He}$ detection efficiency, the results were analogous to those for the scintillator case. Although these studies used neutron detectors, the directional technique can be applied to all types of penetrating radiation, because it depends only on the partial attenuation of the incident fluence across a segmented detector.

Contents. Chapter 2 describes the experimental layout and examines the presumed $1 / r^{2}$ dependence of the detector count rates on source distance, without the explicit use of detector directionality. Chapter 3 explores the basic segmented-detector directionality, which can be observed directly as differences between the count rates for the different elements. Chapter 4 then pursues a more advanced directionality technique, in which ratios between the count rates are used to determine the gradient of the local radiation field and hence the direction to the radiation source. Chapter 5 adapts the technique to handle moving sources and pairs of stationary detectors, and Chap. 6 examines room-scattering effects for both stationary and moving sources. Chapter 7 provides a summary and the conclusions from this initial study.

\section{EXPERIMENTAL ARRANGEMENT}

Overview. This chapter explains the layout for the measurements, including the multielement detectors, the types of sources, and the basic analysis approach. Much of the discussion concerns the unusual radiation response of the particular detectors used for this study. The analysis focuses on nondirectional measurements similar to those made with single-element detectors, which provide only the variation in count rate as a function of source distance.

Detector Geometry. The central component of the measurements is a set of eight detector elements being evaluated for a new neutron-multiplicity instrument. ${ }^{5}$ Although using these particular detectors causes some problems for the present study, the basic directional technique is still valid. As illustrated by the cross-sectional view in Fig. 2.1, each element is a cylindrical scintillator $10.2 \mathrm{~cm}$ in height and $7.6 \mathrm{~cm}$ in diameter, which is made of BC454* boron-loaded plastic that is surrounded by a layer of BGO (bismuth germanate) scintillator $4 \mathrm{~mm}$ in thickness. The light from both scintillators is collected by a single light pipe and photomultiplier tube (PMT), an arrangement often referred to as a "phoswiched" detector. The PMT is connected directly to a circuit board that includes the bleeder string and preamplifier, and the entire assembly is enclosed in an aluminum housing with an outer

* Bicron Corp., 12345 Kinsman Rd., Newbury, OH 44065. 
diameter of $12.7 \mathrm{~cm}$. To provide directionality, four of these rods are stood upright in a two-by-two array, with each one facing a different quadrant. Because of the outer housings, there are large gaps of about $5.1 \mathrm{~cm}$ between the $7.6-\mathrm{cm}$ diameters of the active elements. For most of the measurements, the set of eight BGO-plus-plastic scintillators was arranged to form two arrays separated by a distance of $1 \mathrm{~m}$, which allows simultaneous measurements to be made for each source position.

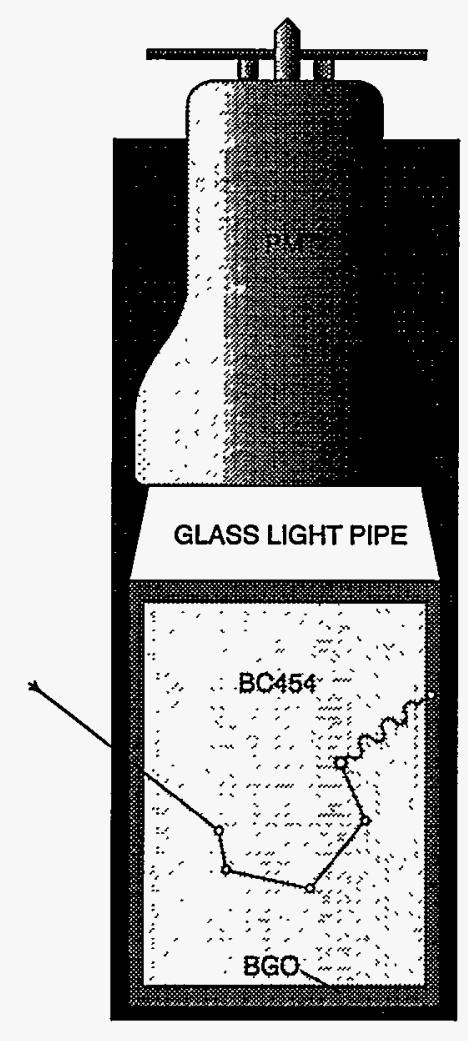

Fig. 2.1. Cross-sectional schematic of an individual detector element, showing the phoswiched BC454 and BGO scintillators connected to a single light pipe and photomultiplier tube (PMT). Also shown is a particle history in which an incident fast neutron repeatedly scatters in the inner plastic scintillator and is absorbed by a low-energy capture reaction, which results in a gamma ray that then interacts with the outer layer of BGO.

Radiation Response. Because of the combination of BGO cladding with a boronloaded plastic scintillator, the detector's radiation response is a complicated mixture of neutron and gamma-ray contributions. In the plastic, gamma rays interact mainly through Compton scattering, with some photoelectric absorption at the lowest energies. In addition, BC454 responds to fast neutrons through n-p scattering and to slow neutrons through the exoenergetic ${ }^{10} \mathrm{~B}(\mathrm{n}, \alpha){ }^{7} \mathrm{Li}^{*}$ reaction, which produces highly ionizing alpha and ${ }^{7} \mathrm{Li}$ ions and usually a 478-keV gamma ray. As with all plastic scintillators, the energy-to-light conversion saturates easily for charged particles, so the response to the recoil protons from the n-p scattering and the alpha and ${ }^{7} \mathrm{Li}$ ions from the boron capture is fundamentally nonlinear. For the $\mathrm{BGO}$, the usual gamma-ray response is combined with an unusual neutron response based on detection of the 478-keV gamma ray from the boron capture in the plastic. Both the fast plastic and the slow BGO light pulses are collected by one PMT and integrated by a single charge-sensitive amplifier, whose compromise integration time gives an output signal that is a nonlinear combination of the two scintillator responses. Given the differences between the interaction mechanisms, light-collection efficiencies, and output pulse shapes, the combined light-output distribution is particularly complicated, as illustrated by the ${ }^{252} \mathrm{Cf}$ spectrum in Fig. 2.2. The light-output scale is based on "equivalent electron" energy, or $\mathrm{keV}_{e e}$, which 
is calibrated empirically using minimum-ionizing electrons, that is, photoabsorption peaks in the BGO and Compton edges in the BC454. For the BC454, the charged particles from the neutron capture produce a saturated peak at about $93 \mathrm{keV}_{e e},{ }^{5}$ and the $478-\mathrm{keV}$ capture gamma ray produces a Compton edge at $311 \mathrm{keV}_{e e}$. As shown in the figure, summing the light outputs from these interactions shifts the edge upward to $404(=311+93) \mathrm{keV}_{\mathrm{ee}}$. For the BGO, the unscattered gamma rays give a photopeak at $478 \mathrm{keV}$, which occurs in the phoswiched output at a lower value because of optical attenuation in the thin BGO crystal. Again, the observed peak is shifted upward by $93 \mathrm{keV}_{e e}$, and the result appears near $250 \mathrm{keV}_{e e}$. Thus, the pulse-height distribution from even a basic neutron source contains a set of capture peaks and edges near light outputs of $100 \mathrm{keV}_{e e}, 250 \mathrm{keV}_{e e}$, and $400 \mathrm{keV}_{e e}$. These peaks are superimposed on a broad continuum from the proton and electron recoil energies.

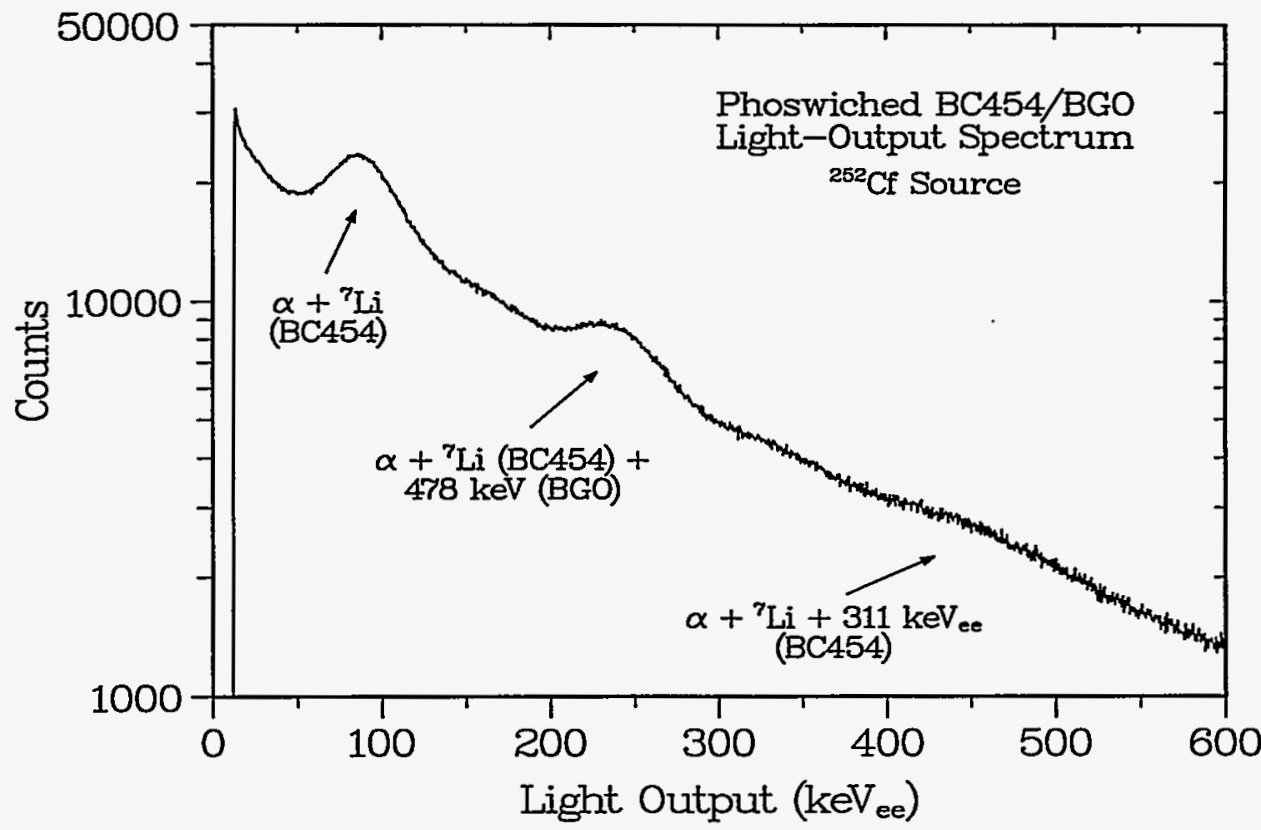

Fig. 2.2. Light-output spectrum for a ${ }^{252} \mathrm{Cf}$ fission neutron source as measured with a phoswiched BC454/BGO scintillator.

Multi-Hit Events. For each of the eight detectors, the preamplifier output from the phoswiched scintillators is fed into a lower-level discriminator, whose setting is referenced to the $\sim 100-\mathrm{keV}_{e e}$ capture peak in the spectrum. Thus, despite the detector's complex radiation response, its only output is a simple logic pulse, which is combined with those from the other detectors into a single output stream. As in standard neutron-multiplicity instruments, ${ }^{6}$ the time correlations between pulses in this output stream are analyzed using a commercial shift-register ${ }^{7}$ unit, which is controlled by a modified version of the neutronmultiplicity program ${ }^{8}$ NCC, both of which were developed by LANL's Safeguards Science and Technology Group (NIS-5). The output of the NCC program includes the count rates from the eight individual rods and the rates of singly, doubly, and triply coincident events. In our case, a multi-hit event is recorded if more than one pulse arrives within a time interval of $5 \mu \mathrm{s}$. The most obvious mechanism would be the simultaneous detection of two different neutrons or gamma rays from the same nuclear fission. Such "true" source 
multiplicities are extremely unlikely in our measurements because of our small detector area and low detection efficiency. For example, at a distance of $10 \mathrm{~m}$ the product of solid angle and efficiency (that is, the effective area) for a single rod is only about $10^{-6} \mathrm{~cm}^{2}$. Thus, even a source fission rate of $10^{5} / \mathrm{s}$ produces measured coincidence rates that are completely insignificant compared with typical room-background levels of $10 \mathrm{~Hz}$ for doubles and $5 \mathrm{~Hz}$ for triples. A more likely double-hit mechanism would be the scattering of a single neutron or gamma ray between two rods, but our electronics includes active filters that reject most of these events as cosmic-ray backgrounds. As a result, in our data the dominant source of double-hit events is a neutron scatter-and-capture mechanism that is unique to organic scintillators loaded with materials such as lithium or boron. ${ }^{5}$ As indicated in Fig. 2.1, most neutrons at fission energies ( $\sim 1 \mathrm{MeV}$ and above) pass through the outer BGO layer with little or no interaction, and about half then scatter from one or more protons in the inner BC454 plastic scintillator, which produces a single fast light pulse. Most of these neutrons escape from the scintillator, but about $10 \%$ have lost enough energy to become trapped in the plastic by the large probability for $n-p$ and $n-{ }^{12} C$ collisions at low energies. After moderating by repeated scattering from hydrogen and carbon, these neutrons usually encounter $\mathrm{a}^{10} \mathrm{~B}$ nucleus and initiate an $(n, \alpha)$ reaction. The recoil energies of the alpha and ${ }^{7} \mathrm{Li}$ ions are immediately converted into a single light pulse in the plastic; if the capture gamma ray undergoes Compton scattering, its energy is also included in this pulse. If the gamma ray (scattered or not) reaches the BGO, it deposits most of its remaining energy at this point and, in our phoswiched detector, again adds to the same output pulse. An incident fast neutron therefore initiates a complex process that begins with a neutron scattering and proton recoil-which gives a first output pulse - and then terminates in a low-energy capture event-which gives a second pulse. This two-pulse sequence occurs only for neutrons at energies above about $1 \mathrm{MeV}$ and only if the scattered neutron is captured in the detector. The time difference between the two pulses follows the $\sim 2-\mu$ s exponential die-away time for the neutron population in the BC454 scintillator. In short, not only is the light output from the phoswiched detectors a complex combination of radiation responses, but the multiplicity output is also very complicated.

Experiment Layout. The initial set of measurements was performed in the Nondestructive Assay (NDA) Training Area established by NIS-5 in the basement of LANL's CMR (Chemistry and Metallurgy Research) building. As described in Chap. 6, later measurements were made at the NIS-5 facilities at LANL TA-35. The layout for the CMR NDA Training Area is shown in Fig. 2.3. The floor and one long wall of the narrow room are earth-backed concrete; the ceiling and the inside walls are thinner materials with open space on the far sides. Although the room is relatively uncluttered, there are several cabinets that contain lead and polyethylene shielding. The sources were placed at various surveyed locations throughout the room; as indicated in the figure, the detectors were placed on a laboratory bench located opposite the earth-backed wall. Backgrounds were measured by placing the sources in a storage safe or by removing them from the main room. Most of the measurements had durations of $5 \mathrm{~min}$, although the background runs used longer counting times to improve the overall statistical accuracies.

Radiation Sources. Three types of sources were used in the present measurements: two $\mathrm{PuO}_{2}$ neutron/gamma-ray sources, two ${ }^{252} \mathrm{Cf}$ fission sources, and a set of three gammaray reference sources. The $\mathrm{PuO}_{2}$ sources (LANL LAO255C10 and LAO261C10) consist of oxide powder in double-walled containers; their assay information is listed in Table 2.1. The neutron output comes from both ${ }^{240} \mathrm{Pu}$ spontaneous fission and $(\alpha, \mathrm{n})$ reactions on oxygen isotopes; the emission rates for the 255 and 261 sources are $1.5 \times 10^{5}$ and $2.3 \times 10^{5} \mathrm{n} / \mathrm{s}$, respectively. The activities of penetrating gamma rays and $\mathrm{x}$-rays with energies above 

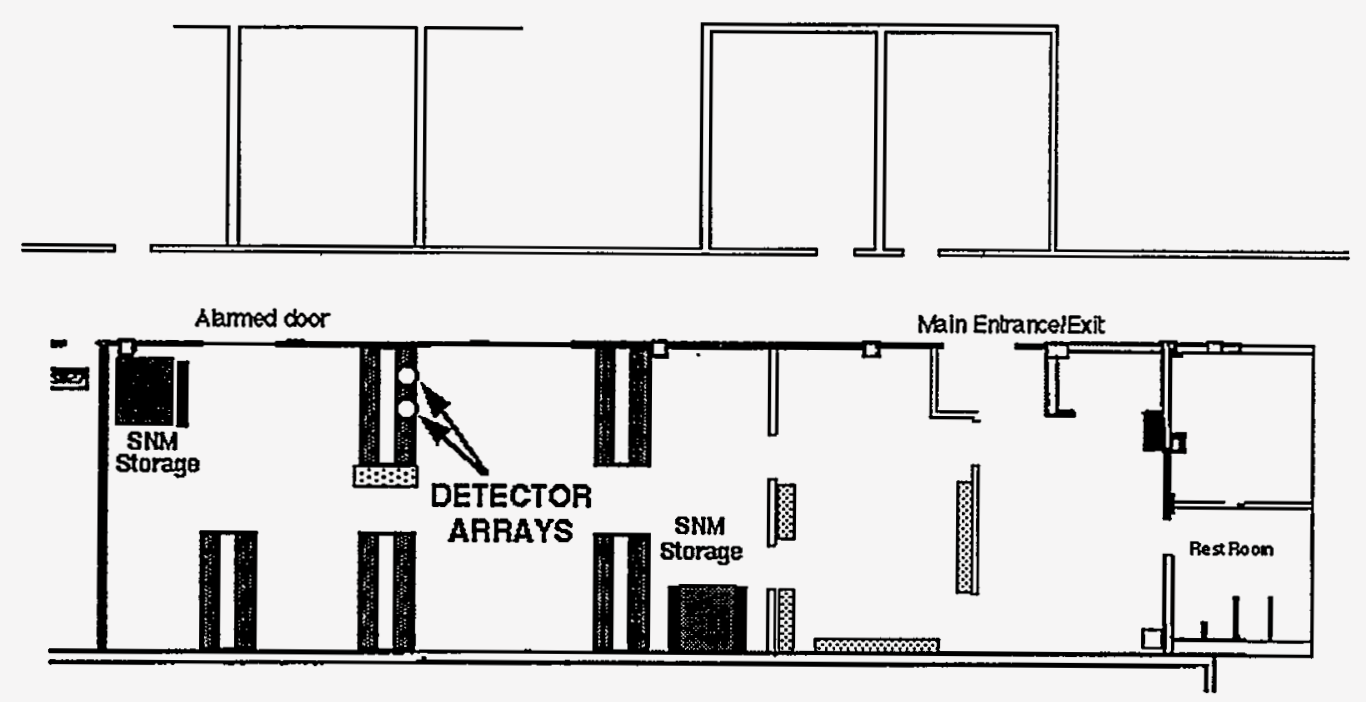

$\left\{\begin{array}{l}\text { Backbobeck } \\ \text { L.bBenches }\end{array}\right.$

Fig. 2.3. Layout of the CMR experimental area, showing the positions of the detector arrays, laboratory benches, and storage cabinets.

$100 \mathrm{keV}$ are about 1000 times greater. The ${ }^{252} \mathrm{Cf}$ sources are LANL CR-10 and CR-11; their neutron emission rates of $2.5 \times 10^{5} \mathrm{n} / \mathrm{s}$ and $6.8 \times 10^{5} \mathrm{n} / \mathrm{s}$ are entirely from spontaneous fission. For our measurements, the ${ }^{252} \mathrm{Cf}$ sources were shielded by $1.25 \mathrm{~cm}$ of lead and $1.25 \mathrm{~cm}$ of polyethylene, so the gamma-ray output was fairly low. The three gamma-ray sources $\left({ }^{137} \mathrm{Cs}\right.$, ${ }^{60} \mathrm{Co}$, and ${ }^{22} \mathrm{Na}$, from Amersham ${ }^{*} 10-\mu \mathrm{Ci}$ set \#188) were chosen to provide gamma-ray lines in the energy range around $1 \mathrm{MeV}$ and a combined count rate similar to those for the $\mathrm{PuO}_{2}$ and ${ }^{252} \mathrm{Cf}$ sources. Details of the ${ }^{252} \mathrm{Cf}$ and gamma-ray sources are given in Table 2.1.

Data Analysis. The measurements were stored both as individual-rod count rates and as total (single-hit), double-hit, and triple-hit rates. Also recorded were the source-todetector distance and the source angle for each run. During each set of runs, background accumulations were periodically made with all sources stored in steel safes lined with $10 \mathrm{~cm}$ of polyethylene (SNM storage in Fig. 2.3). Even this shielding is inadequate for determining the exact background level, however, which can result in some oversubtraction at the largest distances. For critical background runs, the sample source was therefore removed from the training area to provide more accurate values. Another problematic correction concerns the different sensitivities of the individual rods. Because the source direction is determined by comparing the count rates for different elements, the detectors must be matched to avoid an instrumental bias. Unfortunately, the phoswiched arrangement of the two scintillators results in rod-to-rod variations between the BGO and plastic amplitudes, which cannot be adjusted separately. As a compromise, we used a ${ }^{137} \mathrm{Cs}$ source $\left(E_{\gamma}=662 \mathrm{keV}\right)$ to match the gains of the higher-resolution BGO signals, which leaves the plastic-scintillator outputs free to vary by about $15 \%$. To compensate, a further correction was measured by placing a $\mathrm{PuO}_{2}$ source near each rod and determining the normalization factors needed to match

\footnotetext{
* Amersham Buchler GmbH \& Co KG, Postfach 11 49, D-38110 Braunschweig, Germany.
} 
Table 2.1. Reference Sources

Source ID

LAO255C10

LAO261C10

CR-10

CR-11

Cs

Co

$\mathrm{Na}$
Description

$\mathrm{Pu}$ oxide powder (in double steel cans)

$\mathrm{Pu}$ oxide powder (in double steel cans)

Cf oxide powder (in double SS capsules)

Cf oxide powder (in double SS capsules)

Cs ions in resin bead (in Lexan laminate)

Co ions in resin bead (in Lexan laminate)

$\mathrm{Na}$ ions in resin bead (in Lexan laminate)
Isotopes

$16.3 \%{ }^{240} \mathrm{Pu}$

$16.4 \%{ }^{240} \mathrm{Pu}$

${ }^{252} \mathrm{Cf}$

${ }^{252} \mathrm{Cf}$

${ }^{137} \mathrm{Cs}$

${ }^{60} \mathrm{Co}$

${ }^{22} \mathrm{Na}$
Mass or Activity ${ }^{a}$

$543.4 \mathrm{~g} \mathrm{Pu}$

847.9 g Pu

$2.5 \times 10^{5} \mathrm{n} / \mathrm{s}$

$6.8 \times 10^{5} \mathrm{n} / \mathrm{s}$

$11.1 \mu \mathrm{Ci}$

$8.7 \mu \mathrm{Ci}$

$7.8 \mu \mathrm{Ci}$

${ }^{a}$ Activities calculated as of July 1, 1996.

the observed count rates. Such factors are spectrum-dependent and may not be correct for other sources or positions, but their magnitudes were only a few percent.

Total Count Rates. The first experimental results are given in Figs. 2.4a-c, which show the total four-rod count rates for the three types of sources as functions of separation distance. Note that the background count rate has already been subtracted from the data; otherwise, the results would approach a plateau near $550 \mathrm{~Hz}$. Separate sets of measurements are shown for different sources or detector arrays, and simple $1 / r^{2}$ (dotted) and $1 / r^{n}$ (solid) dependences are compared with the results. The measured behavior in each case is approximately $1 / r^{2}$, but the falloff appears to be more rapid for the $\mathrm{PuO}_{2}$ data and less rapid for the ${ }^{252} \mathrm{Cf}$ results. Although an oversubtraction of backgrounds may be partly at fault at the largest distances, the deviations can also result from air scattering and absorption effects. For example, the deviation in the $\mathrm{PuO}_{2}$ case may be related to the relatively large absorption for low-energy gamma rays; for the ${ }^{252} \mathrm{Cf}$ case, fission neutrons from more distant sources can be scattered to lower energies, where the detection efficiency increases rapidly. Detailed analyses and calculations would be needed to check these possibilities. Finally, an important feature of the measurements is their obviously nonstatistical fluctuations, which are probably caused by different detector angles or by scattering and attenuation through cabinets or lab benches. It is this variability that makes the determination of source direction from count-rate measurements at different positions so difficult. For example, for the 10- $\mathrm{Hz}$ signal rate at $15 \mathrm{~m}$ and our background rate of $550 \mathrm{~Hz}$, a counting time of $5 \mathrm{~min}$ gives a statistical uncertainty of almost $20 \%$ in the net rate. Given that the signal is less than $2 \%$ of the background, maintaining this $20 \%$ accuracy requires that the background measurement have a systematic error much less than $1 \%$.

Doubles Count Rates. To summarize the above discussion of single-hit versus multihit events, a scattered or absorbed gamma ray or a captured low-energy neutron always gives a single logic pulse, but a higher-energy fission neutron can produce either one or two output pulses, the latter separated by the few microseconds it takes to thermalize the scattered 


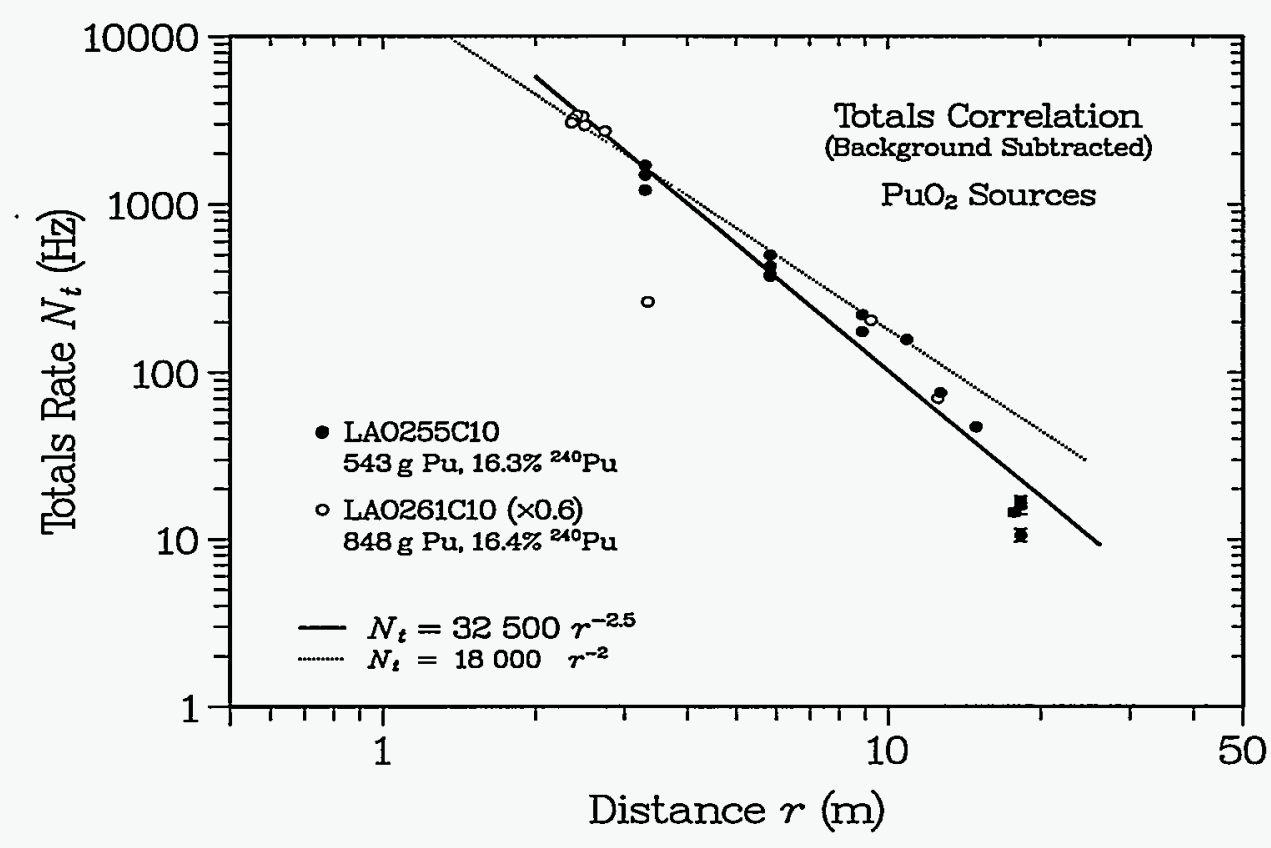

Fig. 2.4a. Variation in the background-subtracted total count rate versus separation distance, as observed with a single detector array and two different $\mathrm{PuO}_{2}$ sources. The solid and dotted lines indicate different power-law dependences of the count rate on separation distance.

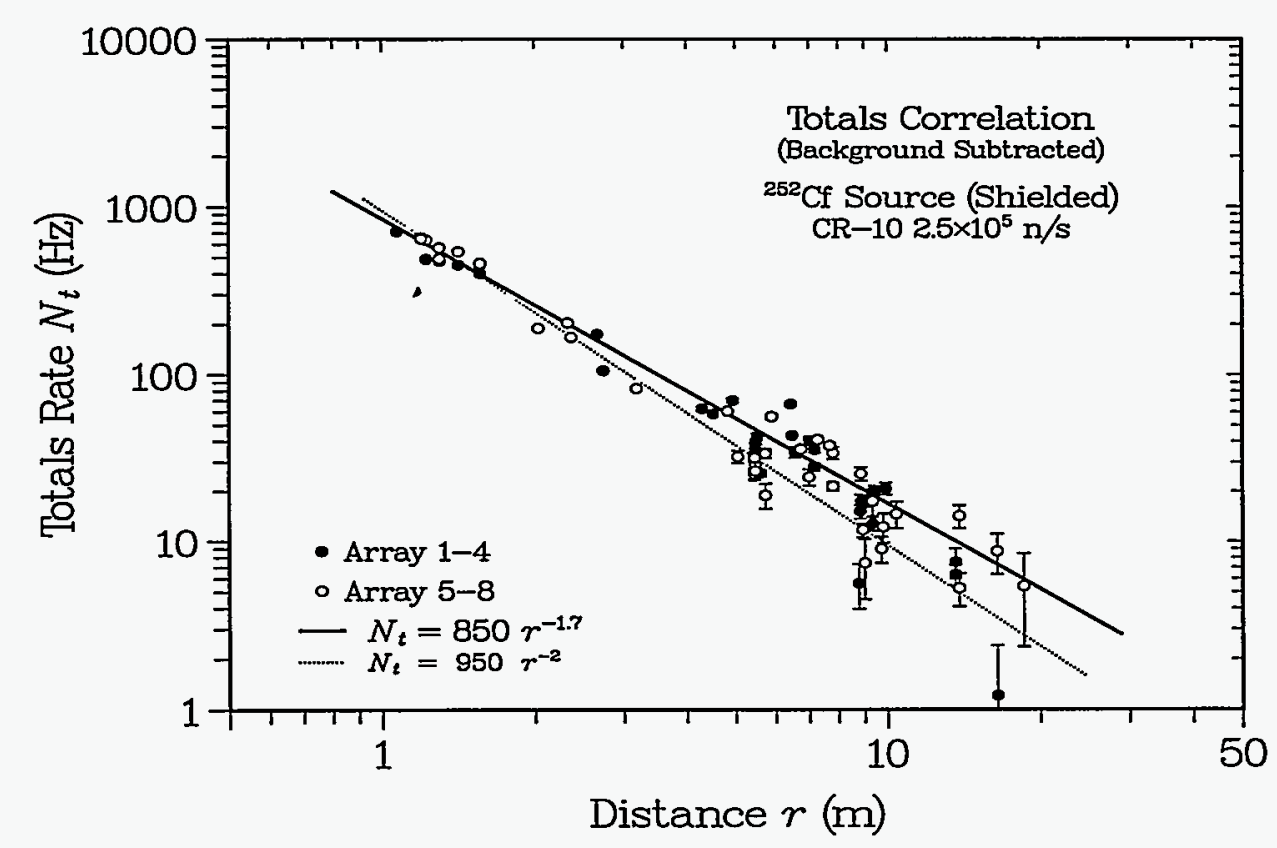

Fig. 2.4b. Total count-rate variation as in Fig. $2.4 \mathrm{a}$, but using the ${ }^{252} \mathrm{Cf}$ fission source.

neutron. Most important, these two-pulse neutron events, even though originating from a single neutron and usually produced in a single detector, will be identified by the shift register as double-hit counts. In agreement with this model, after background subtraction no net 


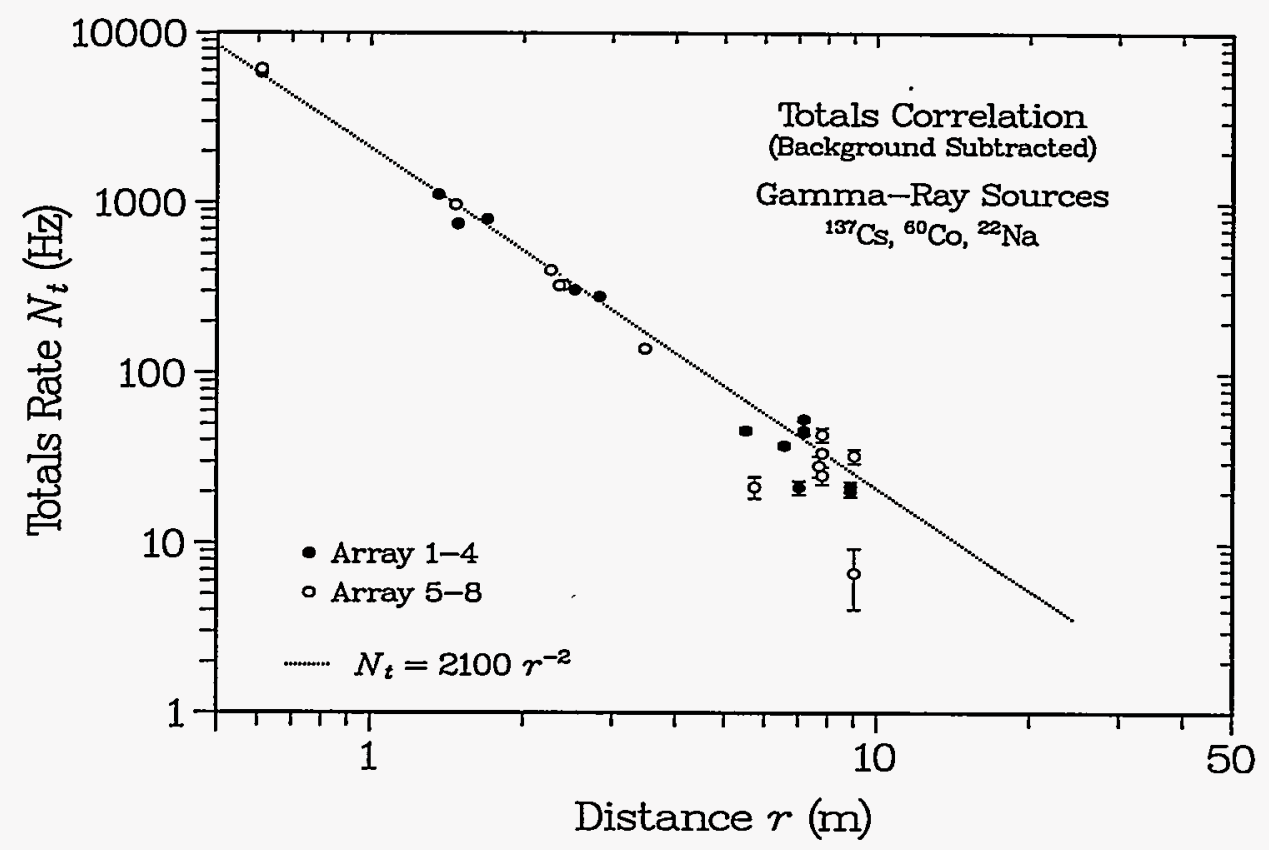

Fig. 2.4c. Total count-rate variation as in Fig. 2.4b, but for the set of three gamma-ray sources.

double-hit count rate was observed for the gamma-ray sources. As indicated in Figs. 2.5a,b, the net doubles rates for the ${ }^{252} \mathrm{Cf}$ and $\mathrm{PuO}_{2}$ sources are seen to vary approximately as $1 / r^{2}$, at least within the statistical uncertainties. Also striking is the 100-fold difference between the totals-to-doubles ratios for the two source types, changing from only $10: 1$ for the ${ }^{252} \mathrm{Cf}$ case to about 1000:I for the $\mathrm{PuO}_{2}$ sources. Again, our assumption is that most of the $\mathrm{PuO}_{2}$ response is caused by relatively low-energy gamma rays, while most of the ${ }^{252} \mathrm{Cf}$ signal comes from high-energy neutrons.

Summary. This discussion of the measurements has described the experiment layout, the detectors and sources, the data analysis, and the behavior of the total and double-hit count rates as functions of source-to-detector separation distance. The main results are the generally, but not exactly, $1 / r^{2}$ count-rate dependence and the importance of background corrections and statistical uncertainties, particularly for measurements at large distances. As stated in the Introduction and discussed in detail in Ref. 2, such count-rate variability often limits the effectiveness of grid-type search procedures.

\section{BASIC DETECTOR DIRECTIONALITY}

Overview. The first evidence for the directionality obtained from the attenuation of radiation across a segmented detector appears as the differences between the count rates for the various elements. In general, the rates on the side closest to the source should be the highest, and those on the opposite side should be the lowest. This chapter tests both this basic assumption and some more advanced alternatives, and then we investigate the utility of this approach for locating various radiation sources. The results afford a review of our previous work ${ }^{1}$ and explain many aspects of the layout for the present experiments.

Angular Dependence. Our first tasks are to define an angular coordinate system in terms of the detector orientation and to examine the angular dependence of the single- 


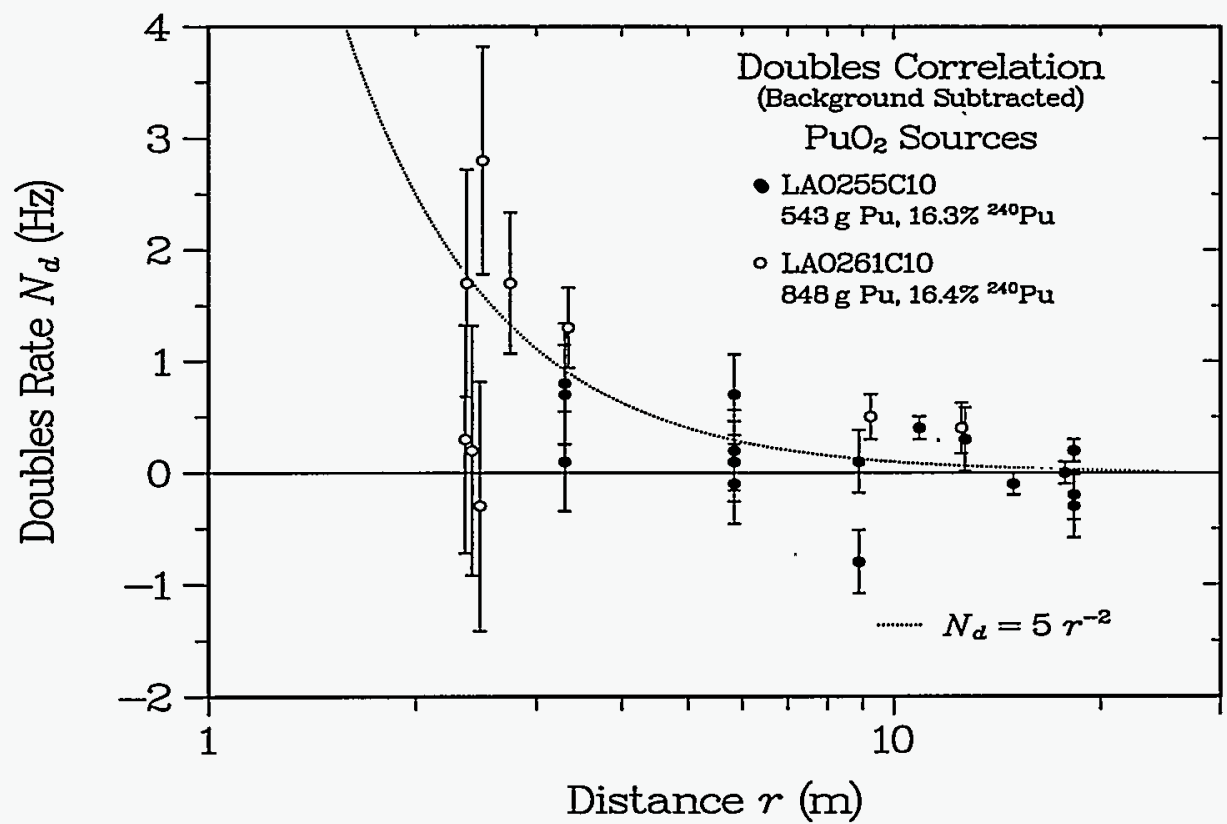

Fig. 2.5a. Dependence of double-hit count rates on distance for the pair of $\mathrm{PuO}_{2}$ sources.

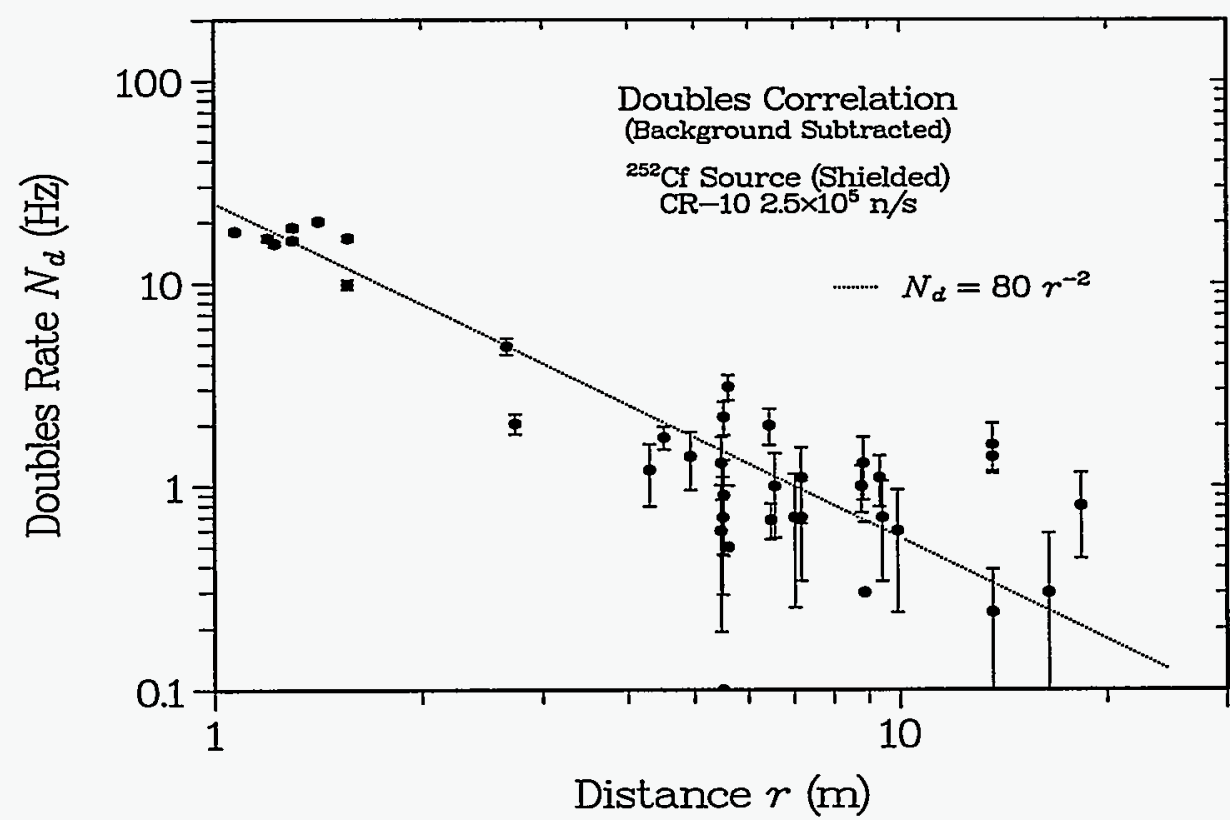

Fig. 2.5b. Double-hit count-rate dependence as in Fig. 2.5a, but for the ${ }^{252}$ Cf fission source.

rod count rates. The small insets in Figs. 3.1a,b show the connection between source coordinates and detector orientations. The segments labelled as rods 1 and 4 make up one pair of opposite elements; rods 2 and 3 form the other pair. The data points shown in the two figures are the count-rate measurements for sources at different locations, corrected for 


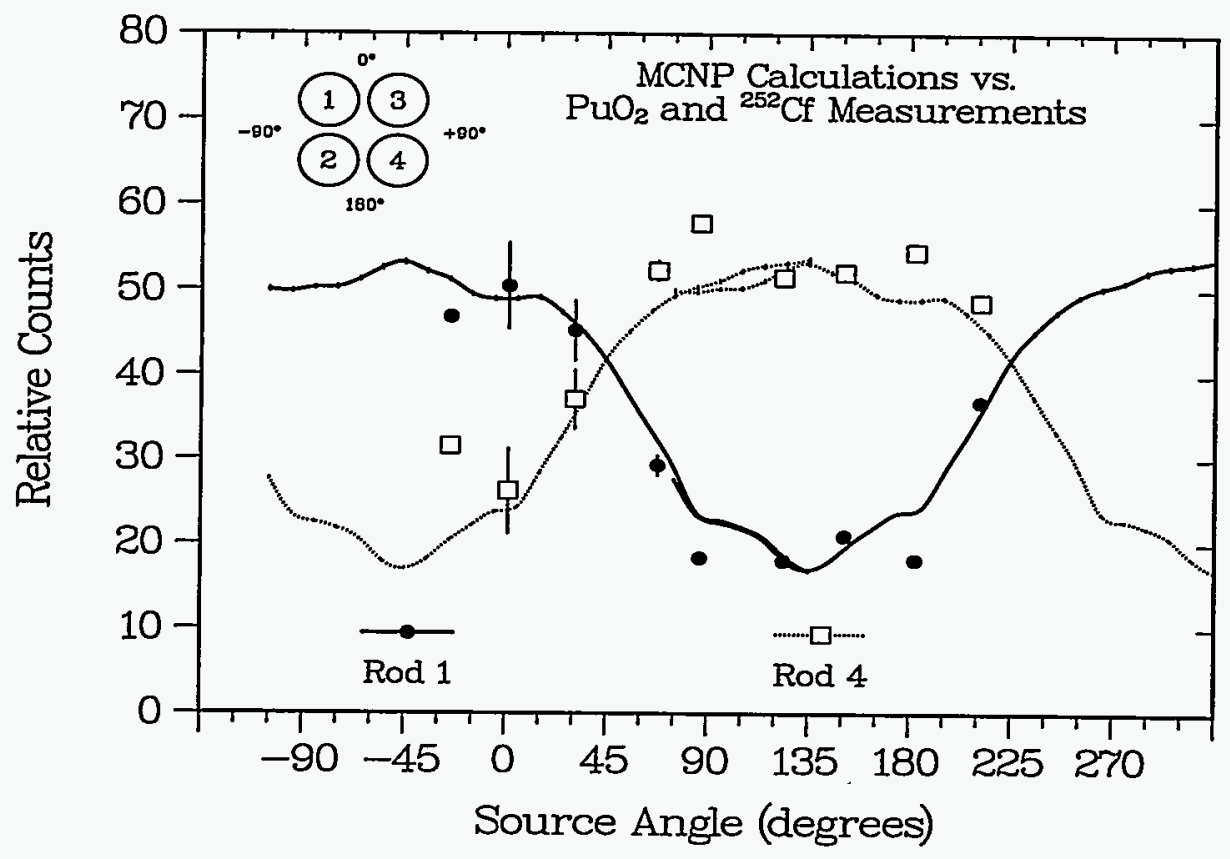

Fig. 3.1a. Variation in individual-rod count rates versus source angle for two opposite elements of a four-rod detector. The measurements shown were obtained by averaging the $\mathrm{PuO}_{2}$ and ${ }^{252} \mathrm{Cf}$ data from the present paper over broad $30^{\circ}$ angle bins.

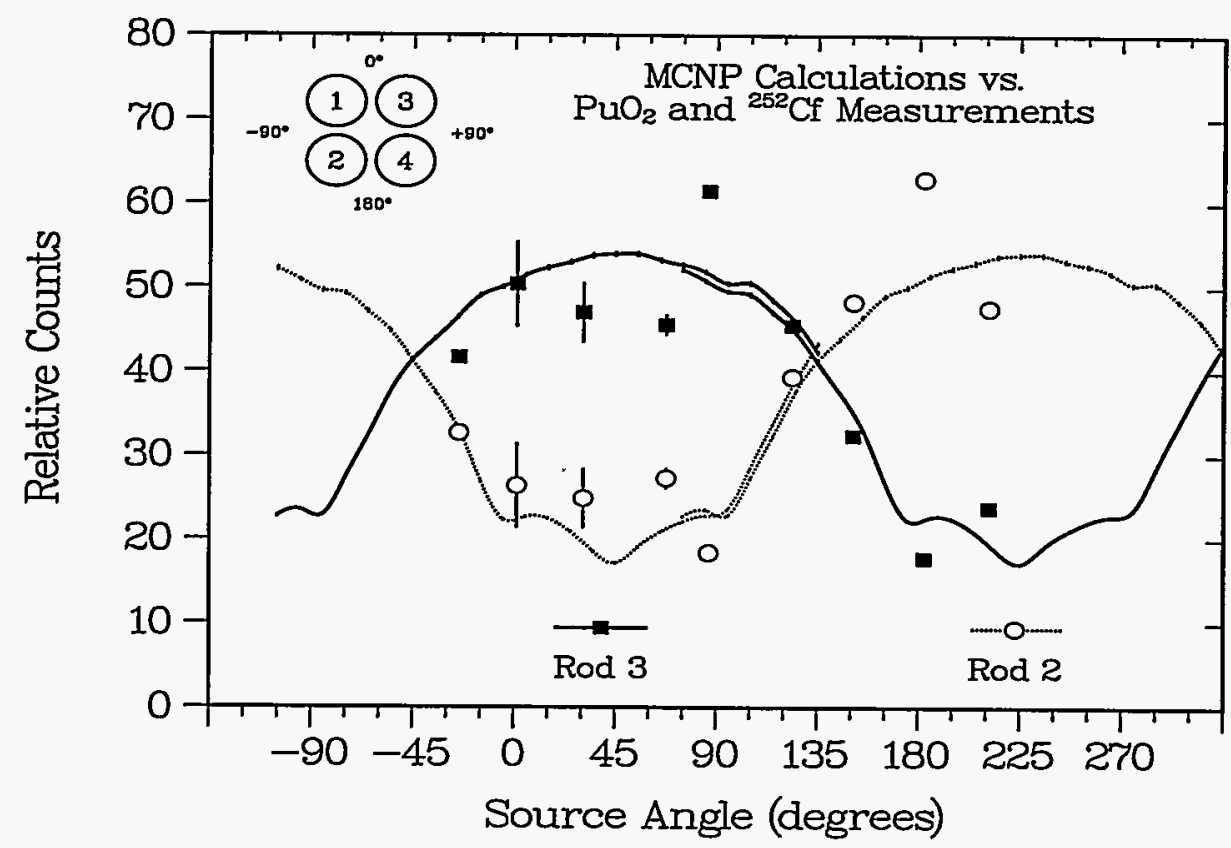

Fig. 3.1b. Angular variation as in Fig. 3.1a, but for opposite rods 2 and 3 instead of rods $I$ and 4.

distance and averaged over $30^{\circ}$ angle bins. Much of the fluctuation in the averages is caused by the uncertainty in the distance correction. Nevertheless, using the angle scale defined by the figure, as a source moves clockwise around the detector from $-45^{\circ}$ to $+45^{\circ}$ and from 
$+135^{\circ}$ to $-135^{\circ}$, the measured count-rate maximum passes in sequence through rods 1,3 , 4 , and 2 , just as expected. The curves are calculations taken from Ref. 1 for an unshielded four-rod detector; they have been shifted horizontally by multiples of $90^{\circ}$ and overlapped to cover the entire angular range. Within the scatter in the data, the calculations generally reproduce the roughly 5:2 ratio in the angular peak-to-valley behavior of the measurements, even though the detector geometries and compositions are quite different in the two cases. These results confirm that directional information does exist in the measurements and testify to the generality of the attenuation technique.

Active Collimation. The next step in exploring the directional concepts described in the previous reports is to consider the use of active collimation obtained by correlating the count rates in different rods. The goal in this case is to develop a detector that is sensitive only in specific directions, but without requiring bulky collimation. In effect, instead of using heavy shielding to prevent radiation from reaching the detector from the rear, the count rates in the rear segments are used to compensate for the leakage into the front segments. Reference 1 discusses this technique in detail and describes the resulting instruments as "one-sided" detectors. For example, given the 5:2 ratio between opposite rods 1 and 4 in Fig. 3.1a,b, multiplying the rod- 4 count rate by 0.4 and subtracting the result from the rod-1 count rate should give a corrected rod- 1 distribution that goes to zero at the rod-4 position. Of course, given the symmetry of the segmented array, any one of the four sides can be selected in software, so similar relations exist for the other three quadrants. The collected "opposite-rod" results are shown in Fig. 3.2a, and Fig. 3.2b shows results for a similar algorithm that groups the four rods two-by-two into front and back "adjacent-rod" pairs. Figures 3.2c,d show the same distributions, but this time plotted in polar coordinates to make it easier to visualize the angular patterns. The measurements and their uncertainties are shown as radial bars, with dotted lines indicating negative values. Again, the curves are calculations adapted from Ref. 1 . In general, the agreement is very good except at the gaps between adjacent rods $\left(0^{\circ}\right.$ and $-90^{\circ}$ in Fig. $3.2 \mathrm{a}, 0^{\circ}$ and $180^{\circ}$ in Fig. $\left.3.2 \mathrm{~b}\right)$. At these angles, the incident radiation can pass between the rods almost unattenuated, so the subtraction becomes very sensitive to scattering and alignment effects. This difficulty was also encountered and discussed in Ref. 1, resulting in the suggestion that small shims be placed in these gaps to reduce the crosstalk at these angles. No such shims were used in the present measurements, so we cannot confirm their effectiveness.

Directional Effectiveness. The intent of active collimation is to measure and correct for the fluence incident from the rear of the detector, so that scattered radiation has less effect on the measurements for the forward direction. In our case, comparing the different techniques requires evaluating their success at determining the correct source direction. A simple approach is to assign.the source to the angular quadrant that faces the detector element with the highest count rate. For example, if rod 4 has the highest rate, then the source is assumed to be in the quadrant between $+90^{\circ}$ and $+180^{\circ}$, which faces rod 4 . Figures 3.3a,b show the comparisons between results with the original segmented detector and the two one-sided approaches. The room layout from Fig. 2.3 has been rotated to align the $0^{\circ}$ source angle with the top of the page, and the plotted points show the actual source locations. The different symbols indicate the quadrant assignments; the correct results are shown in the figures' legends, with the pattern rotated by $+45^{\circ}$ for the adjacent-rod case. With perfect accuracy, all points in each quadrant would be plotted with the same symbol. In Fig. 3.3a, the values in the boxes indicate the fraction of the points for which the correct assignment was made. In some situations, ${ }^{2}$ the increase in information content provided by directionality can be offset by the decrease in statistical accuracy associated with subtracting 


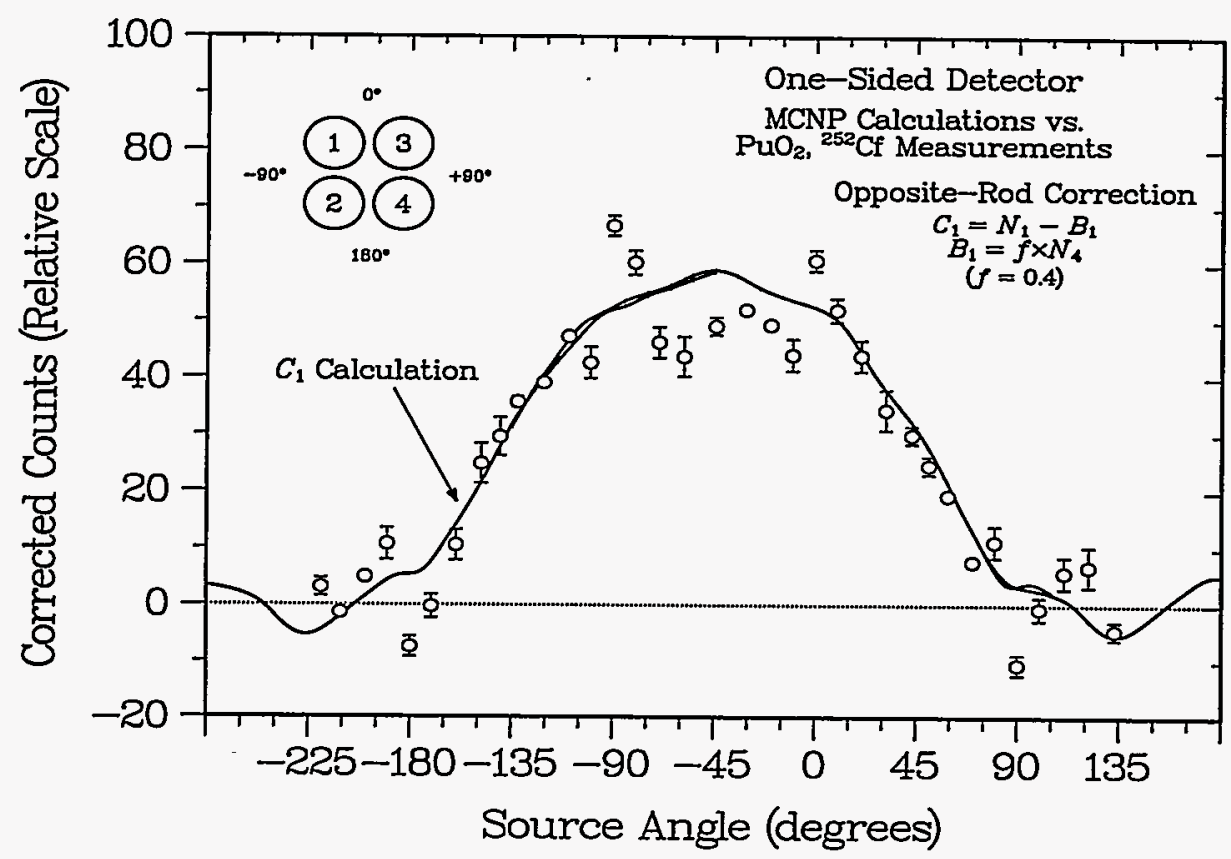

Fig. 3.2a. Effectiveness of electronic collimation in providing a directional detector, using only the differences between the count rates for opposite elements of a four-rod detector package.

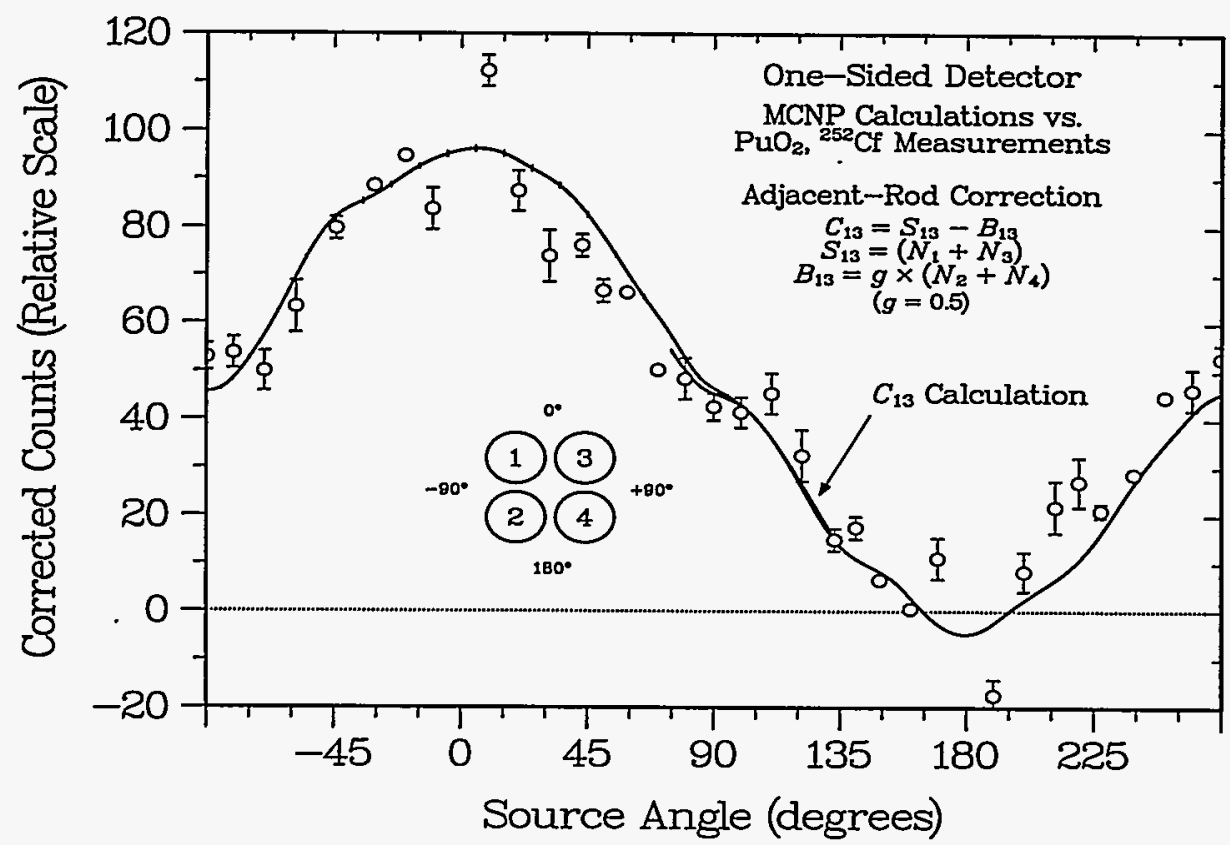

Fig. 3.2b. Directionality as in Fig. 3.2a, but using the two rear rods of a four-rod array to provide an adjacent-rod correction to the count rates for the two front rods.

the different count rates. The result is no net gain-or even a loss-in the angular accuracy for a particular technique. Given that in a random selection only $25 \%$ of the locations in Fig. 3.3a would be correct, the use of a segmented detector provides a clear improvement, 


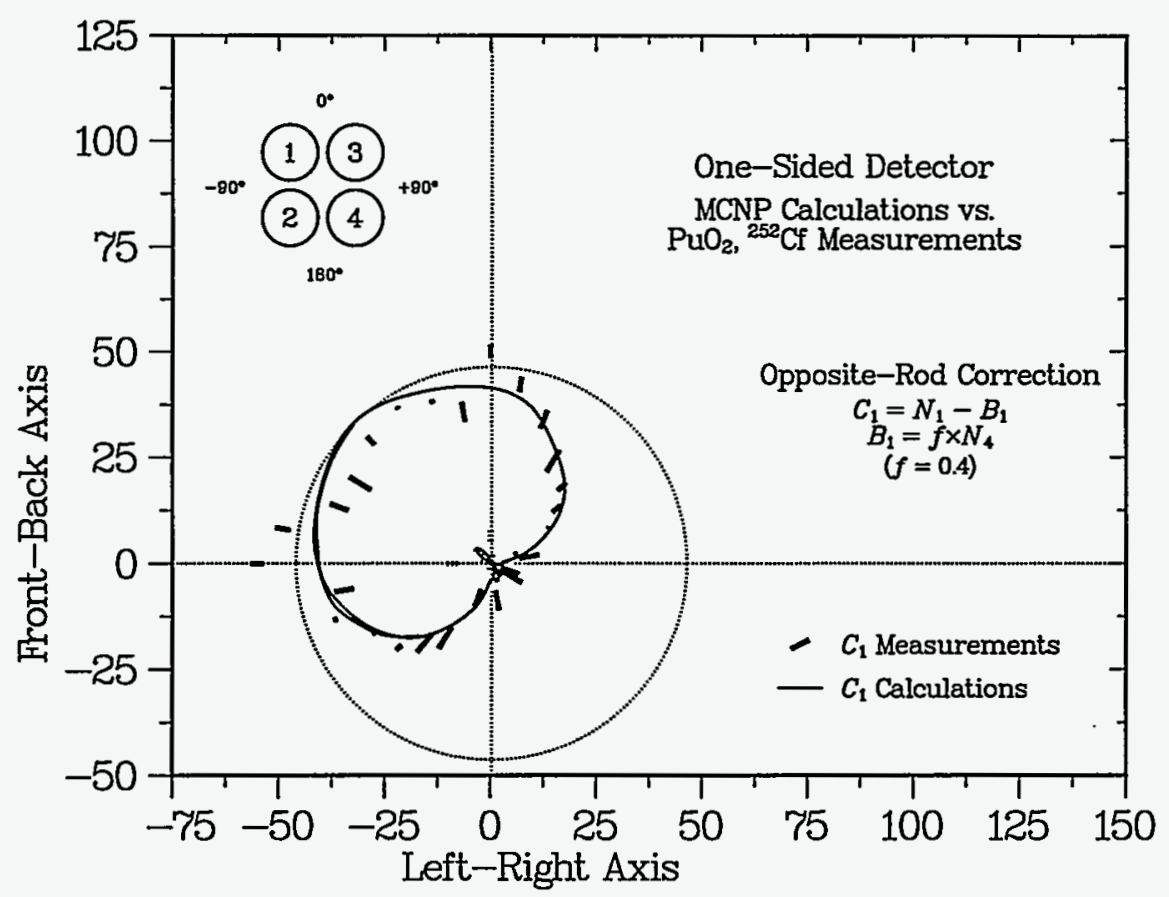

Fig. 3.2c. Polar representation of the opposite-rod measurements and calculations shown in Fig. $3.2 \mathrm{a}$.

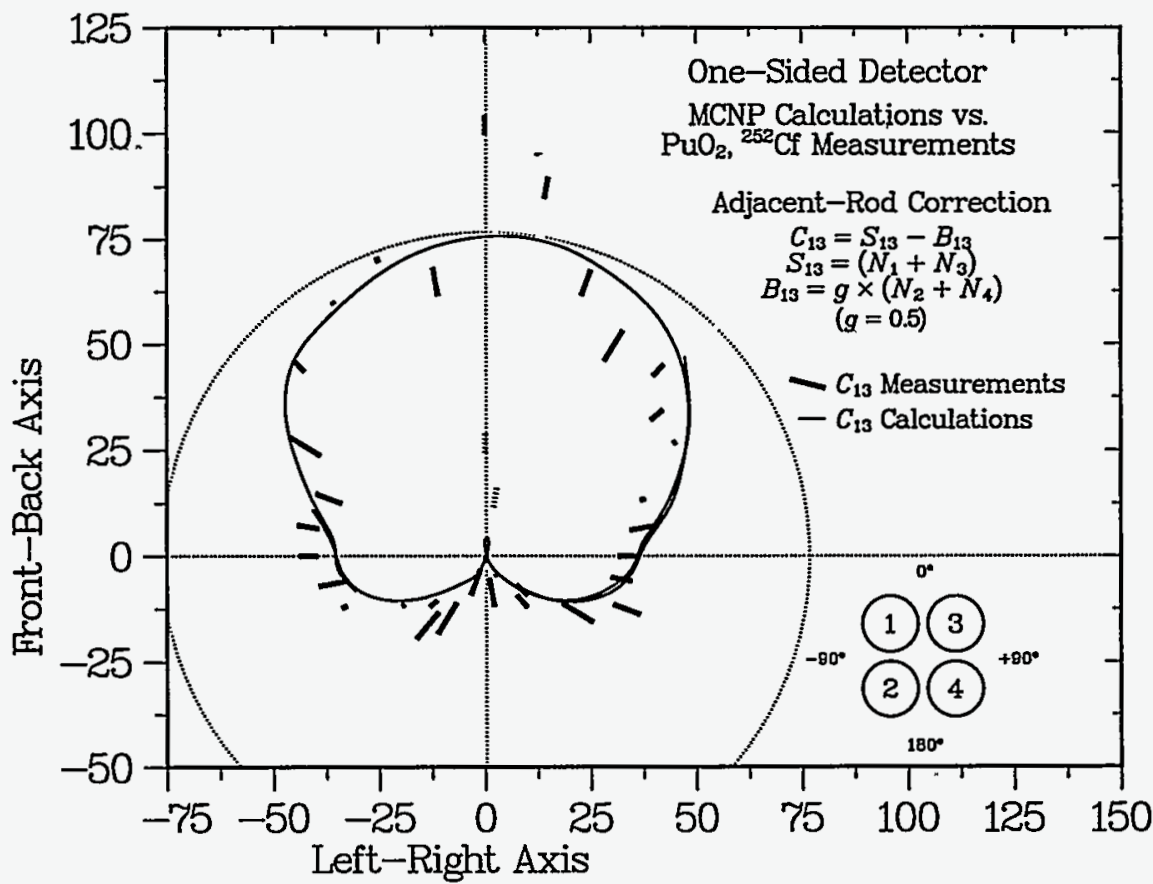

Fig. 3.2d. Polar representation of the adjacent-rod measurements and calculations shown in Fig. $3.2 \mathrm{~b}$.

with the adjacent-rod case attaining an impressive $81 \%$ success rate for the total of 103 runs. To reduce the effect of small differences between the count rates, Fig. 3.3b includes only those cases where the highest and next-highest count rates differ by at least three standard 


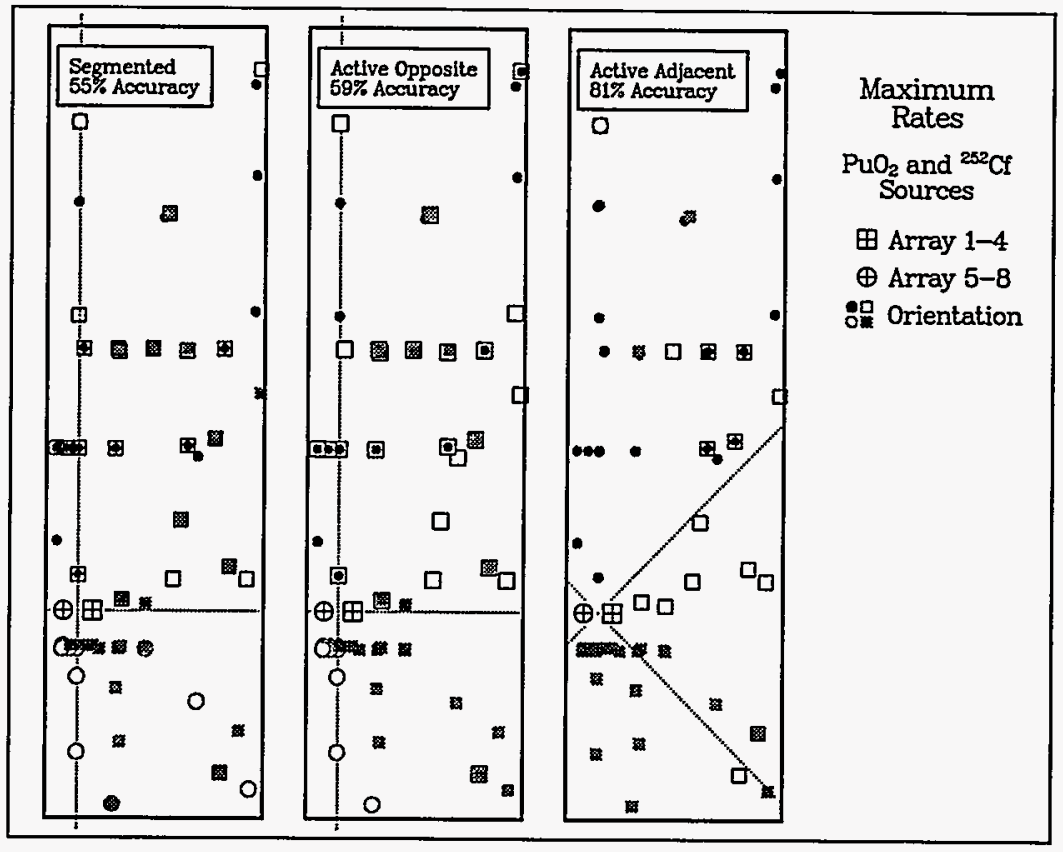

Fig. 3.3a. Comparison between the basic directionality for uncollimated segmented arrays, as determined by assigning the source direction based on the highest count rate for the four rods. The three panels show results for a simple segmented array versus two one-sided approaches that use oppositerod and adjacent-rod active collimation.

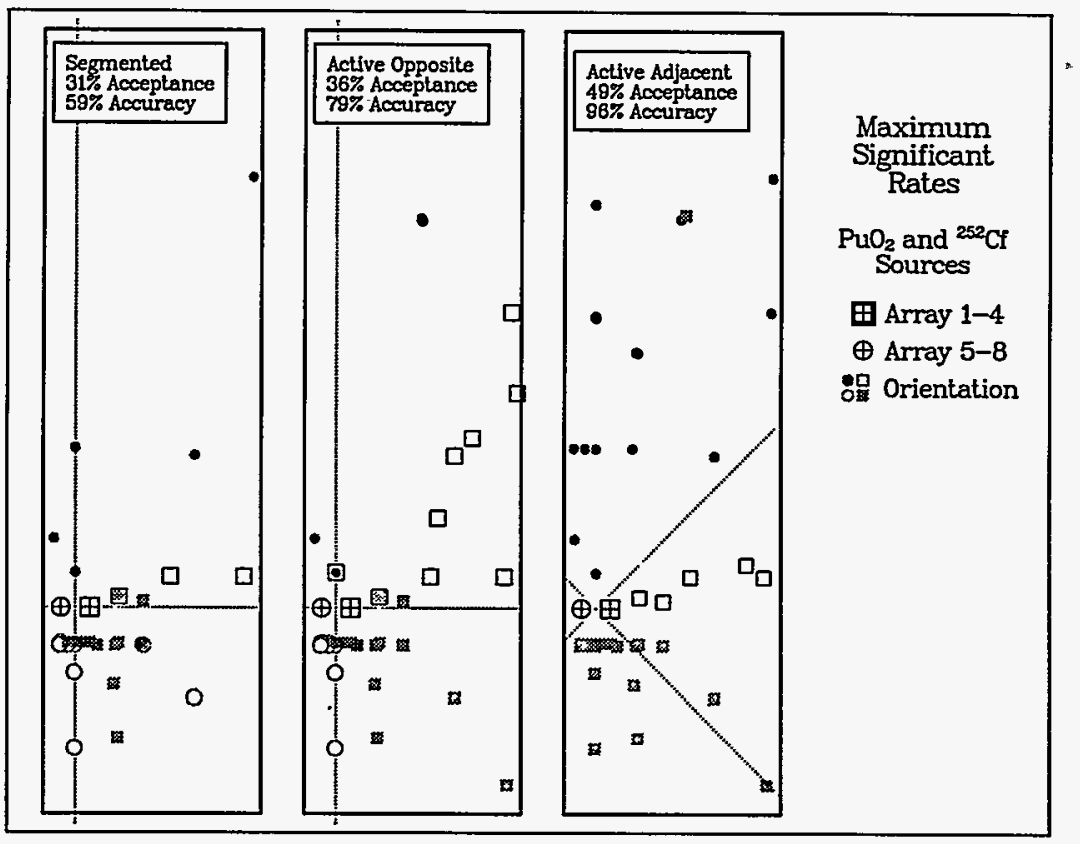

Fig. 3.3b. Comparison as in Fig. 3.3a, but with the requirement that the two highest count rates differ by at least three standard deviations.

deviations. The inset boxes now include the fraction of cases that meet this three-sigma criterion. Because this requirement virtually eliminates the statistical basis for an incorrect 
assignment, the remaining failures must be attributed to systematic effects, presumably room-scattered backgrounds. As expected, active collimation reduces this background and significantly improves the angle accuracy. Furthermore, the narrower angular acceptance heightens the contrast between adjacent quadrants, which enhances the directional signal and extends the detector's useful range-thereby accounting for the improved acceptance. Given that the effectiveness of each approach is the product of the rates for acceptance and success, the net improvements for the two active techniques are factors of 1.5 and 2.5 . This difference is itself significant, in that the adjacent-rod approach provides twice as much active detector volume as the opposite-rod one, in which the two side rods serve merely as shielding material. Finally, the excellent accuracy of the adjacent-rod approach can also influence its application in a search procedure: for the almost $50 \%$ of the assignments that are identified as statistically significant, the assigned direction is virtually $100 \%$ certain to be correct. This level of confidence cannot be obtained without the use of active collimation.

Summary. These simple measurements and analyses have shown that the attenuation of radiation across a segmented detector produces clear variations in the count rates for different segments as the source is moved around in angle. These count rates can be used directly to deduce an approximate direction to the source, and more complicated countrate combinations can be used to produce actively collimated detectors whose net response is limited to a certain angular range. Such techniques can lead to better determination of source directions by improving the angular resolution and by eliminating the effect of scattered backgrounds. In addition, the excellent results for the adjacent-rod approach suggest the importance of making best use of the available detector volume. Although more elaborate one-sided algorithms could be developed, the next chapter follows this suggestion to develop a more sophisticated approach that provides even greater capability.

\section{ADVANCED DIRECTIONAL TECHNIQUES}

Overview. An advanced version ${ }^{1,2}$ of the one-sided detectors in the previous chapter can be developed using a concept adapted from optical applications. From this viewpoint, a radiation field combines a fully directional source with a random background, and a specialized detector is used to determine the relative magnitudes of the two contributions. The directionality of the field is given by the vector polarization $P$, whose magnitude expresses the ratio of signal $(P=1)$ to background $(P=0)$ and whose direction indicates the average source position. A detector's ability to respond to this directionality is referred to as its analyzing power $A$, which can range from perfect $(A=1)$ to zero $(A=0)$. A directional measurement therefore depends on both the polarization $P$ and the analyzing power $A$, as expressed by the product $R=A P$, which defines the observable known as the asymmetry $R$. This conceptual approach, in which the detector analyzes the polarization of the radiation field to produce an observable asymmetry, provides a useful framework for describing the effect of directionality on our experiments.

Mathematical Formalism. For directionality, the central observable in our segmented detector is the count-rate asymmetry $R$, which combines the effects of both the polarization of the field and the analyzing power of the detector. The fundamental quantities are the count rates $N_{i}$ for the opposite elements, whose ratios are used to calculate the $x$ and $y$ components for the measured asymmetry $R$ as follows:

$$
\begin{array}{cc}
r_{x}=N_{+x} / N_{-x}=N_{1} / N_{4}, & r_{y}=N_{+y} / N_{-y}=N_{3} / N_{2} \\
R_{x}=\left(r_{x}-1\right) /\left(r_{x}+1\right), & R_{y}=\left(r_{y}-1\right) /\left(r_{y}+1\right) .
\end{array}
$$




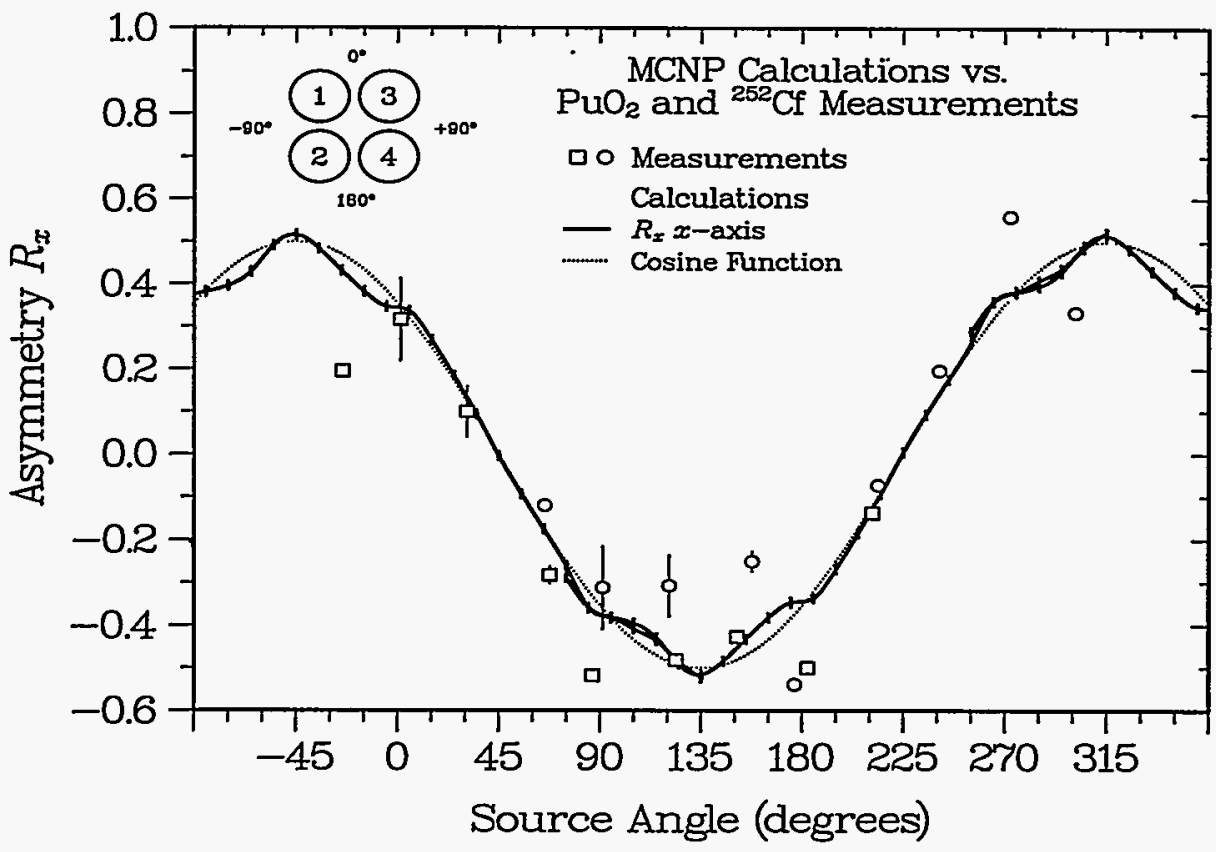

Fig. 4.1a. Comparison between the measured and calculated analyzing powers obtained using the individual-rod results in Figs. 3.1a,b.

The significance of these expressions can be seen in Fig. 4.1a, which shows the behavior of the asymmetry as a function of source angle $\theta$, as obtained from the calculations and measurements shown in Figs. 3.1a,b. The calculated variation in $R_{x}$ is seen to closely follow the cosine dependence expected for a vector component, but the agreement is far from perfect for the measured values of $R_{x}$ (circles) and $R_{y}$ (squares, shifted by $\pi / 2$ ), largely because of the approximate distance correction used in Figs. 3.1a,b. Nevertheless, we can still obtain the approximate relationships

$$
R_{x} \approx R \cos (\theta+\pi / 4), \quad R_{y} \approx R \sin (\theta+\pi / 4),
$$

where we have used the fact that $R_{y}$ is $90^{\circ}$ out of phase with $R_{x}$. These functions lead immediately to two very important expressions for the magnitude $R$ and the direction $\theta$ of the measured asymmetry vector:

$$
\begin{gathered}
R^{2} \approx R_{x}^{2}+R_{y}^{2} . \\
u=R_{y} / R_{x} \approx \tan (\theta+\pi / 4) .
\end{gathered}
$$

The corresponding error analysis takes the fractional errors $\epsilon_{ \pm i}=d N_{ \pm i} / N_{ \pm i}$ from the NCC program's output files and then propagates the values for the two components $d R_{x}$ and $d R_{y}$ as

$$
\begin{gathered}
d r_{x}=r_{x}\left(\epsilon_{+x}^{2}+\epsilon_{-x}^{2}\right)^{1 / 2}, \quad d r_{y}=r_{y}\left(\epsilon_{+y}^{2}+\epsilon_{-y}^{2}\right)^{1 / 2} \\
d R_{x}=2 /\left(r_{x}+1\right)^{2} d r_{x}, \quad d R_{y}=2 /\left(r_{y}+1\right)^{2} d r_{y} \\
\epsilon_{R}=\left(R_{x}^{2} d R_{x}^{2}+R_{y}^{2} d R_{y}^{2}\right)^{1 / 2} / R \\
d u=u\left(\epsilon_{x}^{2}+\epsilon_{y}^{2}\right)^{1 / 2} \\
d \theta=d u /\left(1+u^{2}\right) .
\end{gathered}
$$


The remainder of this chapter examines the validity and applicability of the asymmetry approach. Again, the calculations in Fig. 4.1a agree well with the expected cosine function, but the measurements agree poorly with the calculations - which were, after all, made for a detector array with a different geometry.

Average Asymmetry Behavior. The validity of interpreting $R_{x}$ and $R_{y}$ as the components of an asymmetry vector $R$ depends on observing the appropriate correlations in the measured magnitudes and directions. Initially, we will focus on the angle-averaged data from Figs. 3.1a,b, whose $R_{x}$ and $R_{y}$ components were shown in Fig. 4.1a. First, in Fig. 4.1b we show the results for the asymmetry magnitude $R \approx\left(R_{x}^{2}+R_{y}^{2}\right)^{1 / 2}$ obtained from the data in Fig. 4.1a. The calculated values are relatively constant, with an average magnitude of $0.49 \pm 0.02$; although the measurements have an average value of $0.41 \pm 0.17$, there is a large range of variation. In contrast, Fig. 4.1c shows the corresponding results for the asymmetry direction, where the agreement of both the calculations and the measurements with the actual angles-that is, the cosine dependence-is unquestionably excellent. This contrast, where asymmetry magnitudes behave poorly compared with directions, is a characteristic of the formalism laid out in Eqs. 4.1-4.3. Although the quantities are all calculated using ratios, those for the direction are superratios, that is, ratios of ratios, which provide a higher degree of cancellation for the systematic errors in the measurements. We will see similar behavior throughout our analyses.

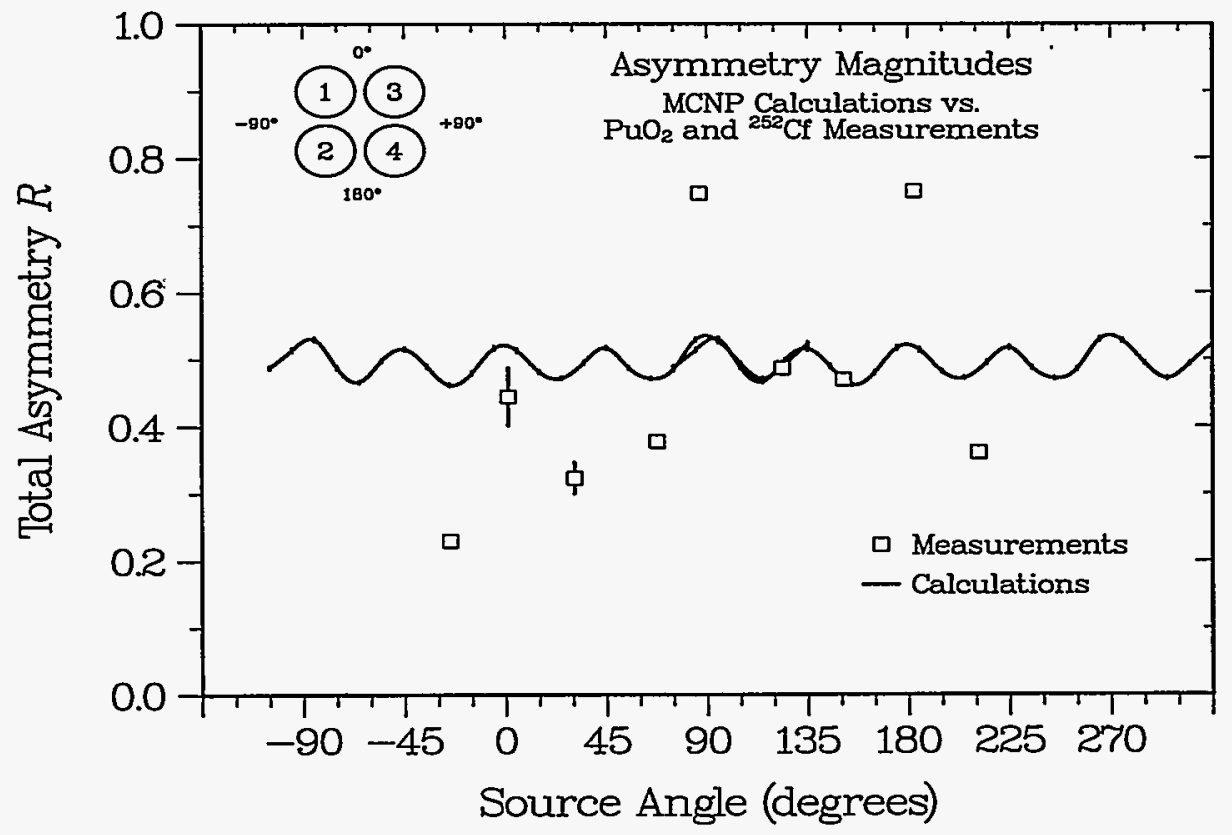

Fig. 4.1b. Comparison between the magnitudes of the measured and calculated asymmetries obtained from the individual-rod results in Figs. 3.1a,b.

Detailed Asymmetry Magnitudes. Based on the results of the angle-averaged data, we now examine the behavior of the individual measurements. In our model, the analyzing power $A$ arises from the internal attenuation across the detector, and the polarization $P$ refers to the external radiation field. As a result, the measured asymmetry $R=A P$ can change either because the value of $A$ changes for different types of radiation or because the 


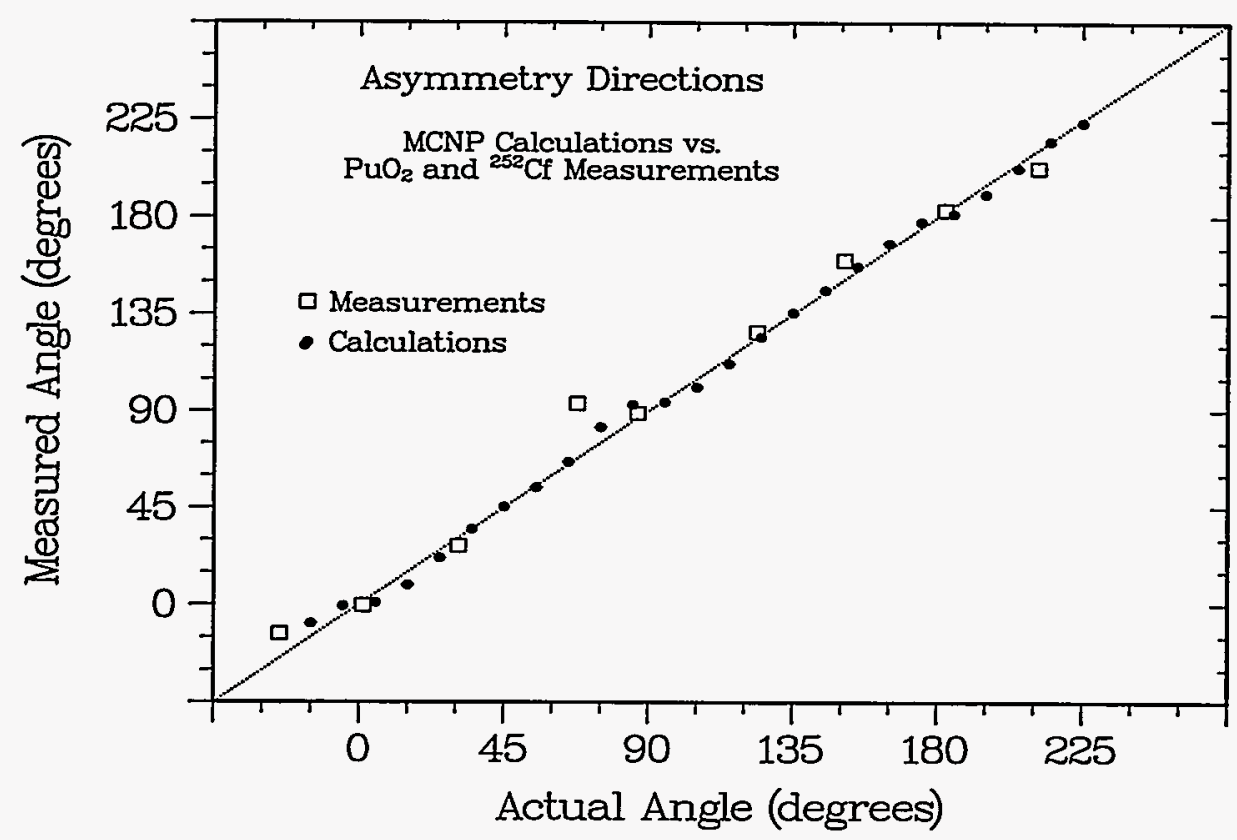

Fig. 4.1c. Comparison between the measured and calculated asymmetry directions obtained using the individual-rod results in Fig. 3.1a,b.

value of $P$ drops as the contribution from room scattering increases with source distance. These predictions are borne out by the results seen in Figs. $4.2 \mathrm{a}-\mathrm{c}$, which show the distance dependence of the measured asymmetries for our three main source types. To emphasize the effect of background contributions, in Fig. 4.2a the asymmetries for the two $\mathrm{PuO}_{2}$ sources are presented without background subtraction. If background subtraction were included, the $R$-values at short distances approach the maximum value of $R=1$. In this situation the incident radiation should be completely polarized $(P=1)$, so $R=1$ implies that $A=1$; that is, the radiation is completely attenuated in crossing the detector. Furthermore, the nearly $30 \%$ fluctuations in the magnitudes far exceed the statistical uncertainties, just as for the count-rate results in Figs. 2.4a-c. This behavior confirms that the radiation from the $\mathrm{PuO}_{2}$ source consists largely of $\mathrm{x}$-rays and low-energy gamma rays, which would indeed produce high, fluctuating attenuations that depend on the details of detector illumination and rodto-rod shadowing within each array. For comparison, the asymmetries for the ${ }^{252} \mathrm{Cf}$ source in Fig. 4.2b and the gamma-ray sources in Fig. 4.2c are shown with background subtraction. Both data sets still fluctuate, but they suggest analyzing powers near $A=0.4-0.5$ and have different dependences on distance. The distance variations for neutrons and gamma rays again agree with our count-rate analyses, and the $A$-values are consistent with the average magnitudes shown in Fig. 4.1b.

Detailed Angle Accuracies. Our next step is the comparison between the actual and measured angle values for the individual measurements, which are shown in Figs. 4.3a-c. For reference, in the room layouts shown in Figs. 3.3a,b of the previous chapter, $0^{\circ}$ is at the top of the figure and the angles increase clockwise. Because of the arrays' placement toward one side of the training area, few measurements could be made at negative angles, and most of those at large distances-and hence with large uncertainties-were located near $0^{\circ}$. In general, the agreement is within the larger of either the uncertainties or $20^{\circ}$. The 


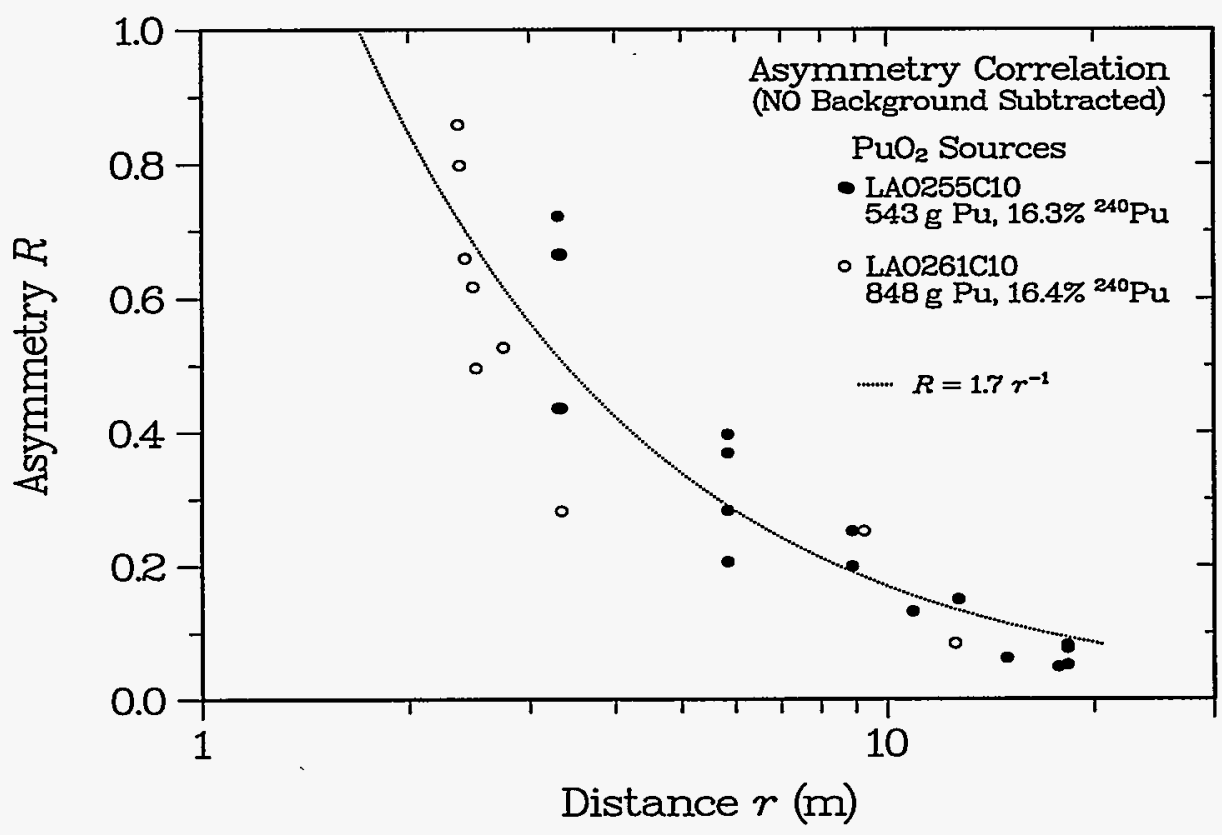

Fig. 4.2a. Count-rate asymmetry as a function of separation between the four-rod detector array and the $\mathrm{PuO}_{2}$ sources. Note that backgrounds have not been subtracted.

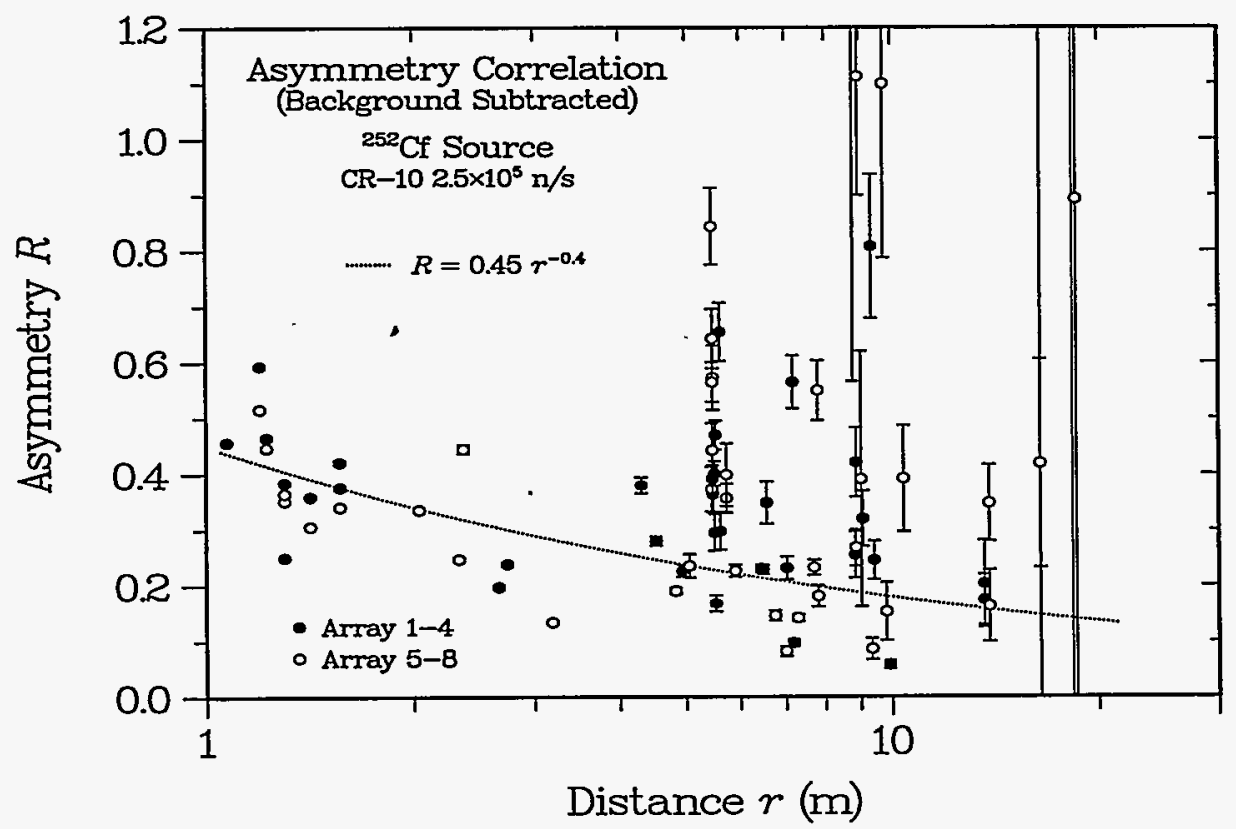

Fig. 4.2b. Asymmetry versus separation as in Fig. 4.2a, but with background subtracted and using the ${ }^{252} \mathrm{Cf}$ fission source.

exceptions suggest that systematic errors may exist, but they do not appear to be angledependent. In Figs. 4.4a-c we further examine the angle accuracy by plotting the distance dependence of the angle error, that is, the difference between the actual and measured 


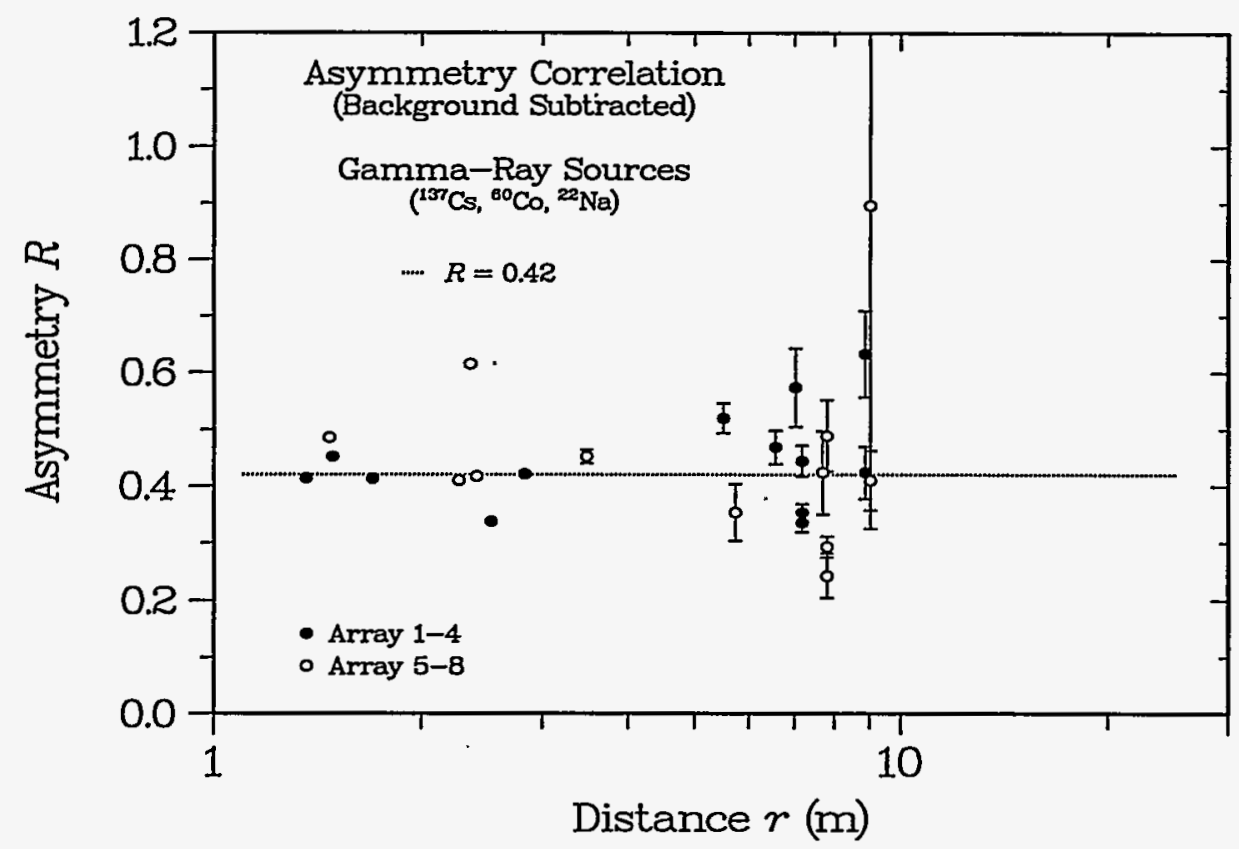

Fig. 4.2c. Asymmetry dependence as in Fig. 4.2b, but for the set of three gamma-ray sources.

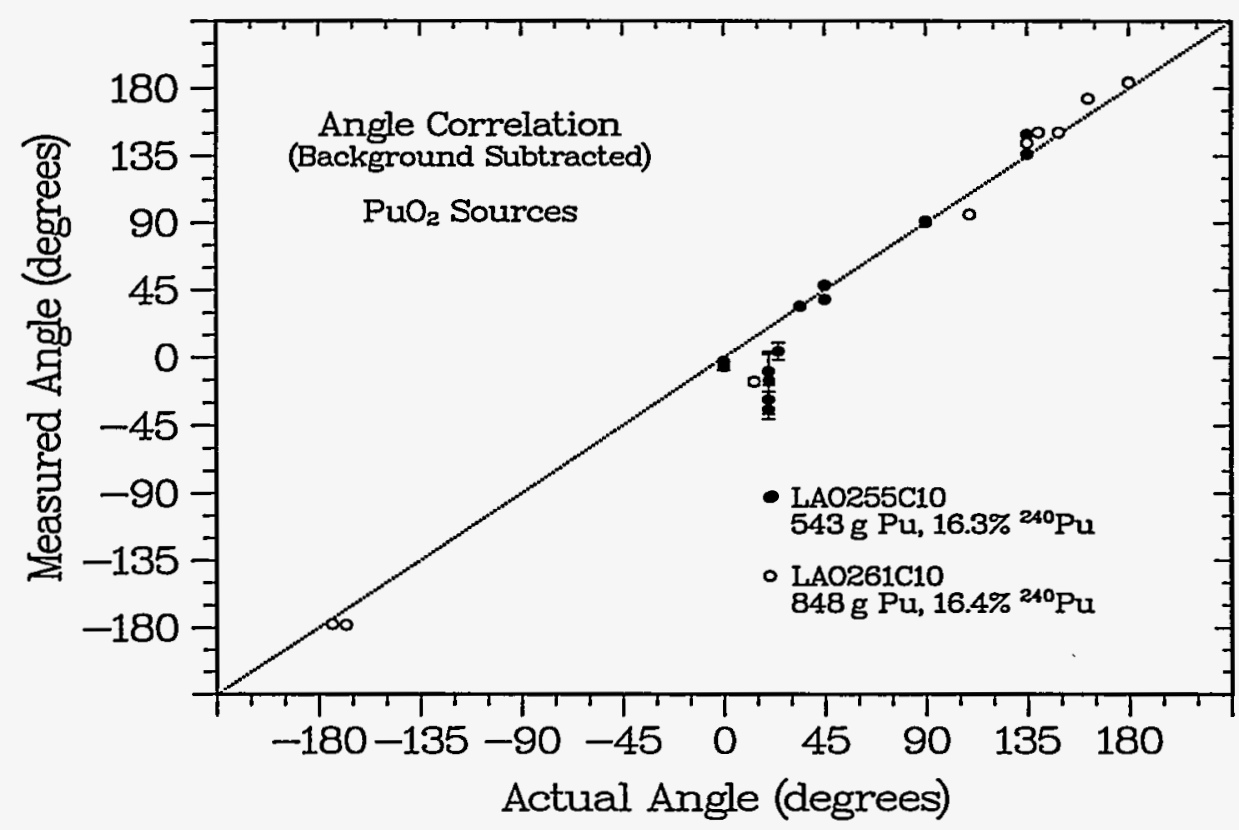

Fig. 4.3a. Comparison between the actual angles for a pair of $\mathrm{PuO}_{2}$ sources and the directions calculated using count-rate differences for one of the fourrod arrays.

angles. For the $\mathrm{PuO}_{2}$ sources in Fig. 4.4a, the high count rates and large asymmetries lead to small statistical uncertainties, but there appears to be a systematic error of about $\pm 10^{\circ}$. 


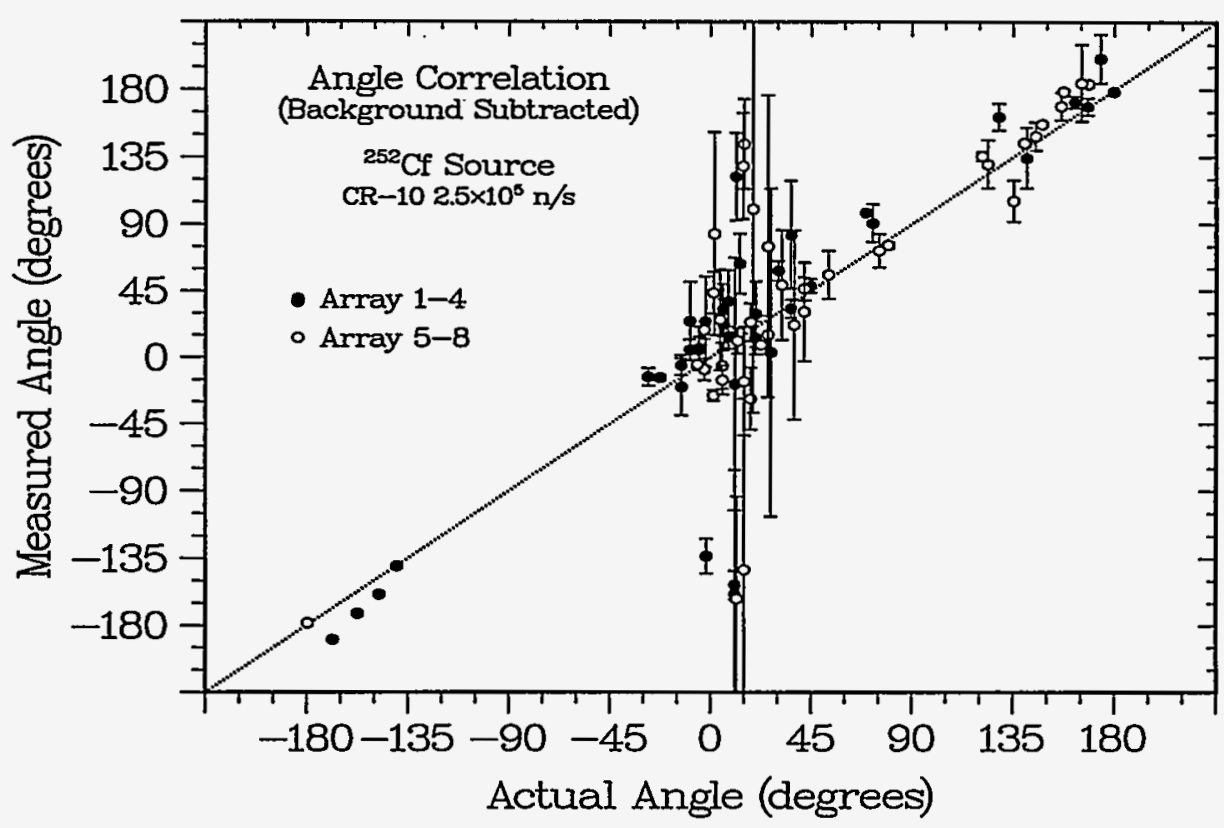

Fig. 4.3b. Comparison between the actual and calculated directions as in Fig. 4.3a, but for the ${ }^{252} \mathrm{Cf}$ fission source and using both four-rod arrays.

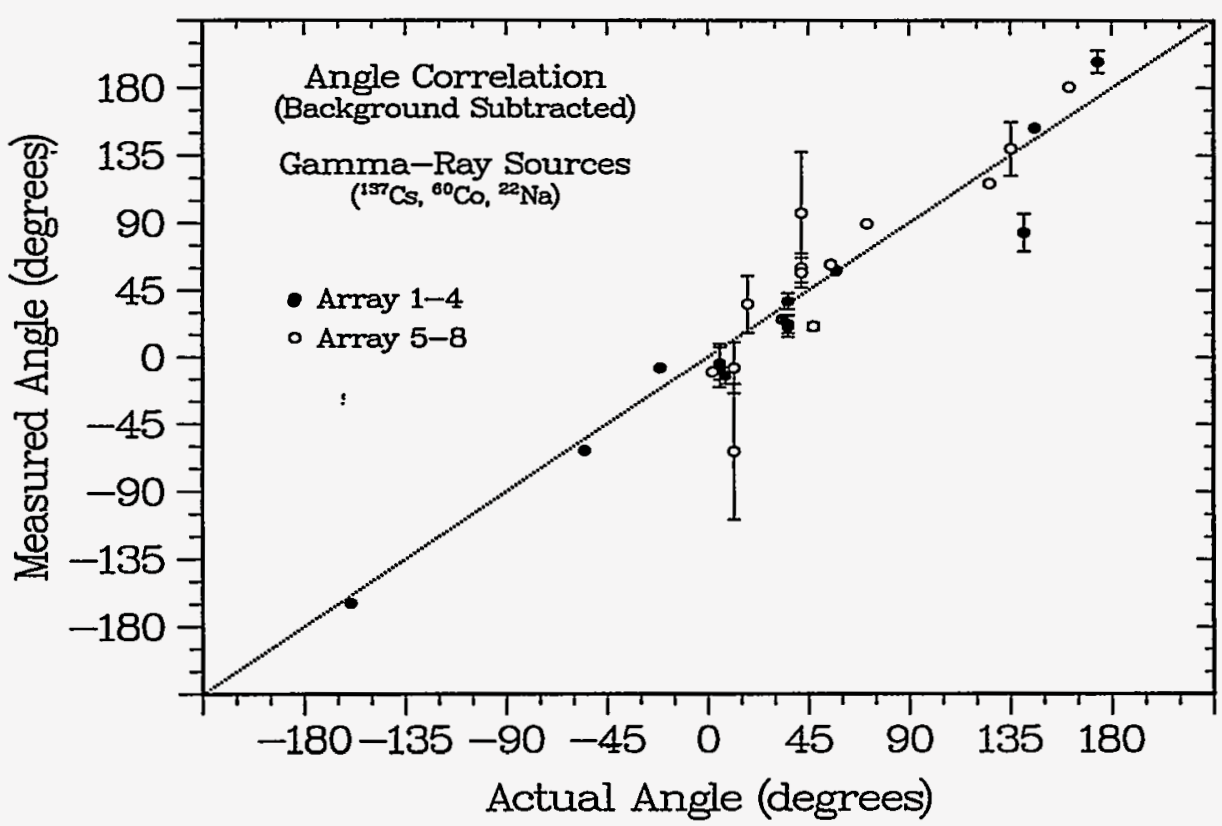

Fig. 4.3c. Comparison between the actual and calculated source angles as in Fig. $4.3 \mathrm{~b}$, but for the set of three gamma-ray sources.

For the ${ }^{252} \mathrm{Cf}$ and gamma-ray sources in Figs. $4.4 \mathrm{~b}, \mathrm{c}$, statistical uncertainties dominate the accuracies at large distances, but the apparent systematic effects are particularly severe at short distances and give angle errors of about $\pm 20^{\circ}$. One explanation for the short-range behavior is rod-to-rod shadowing, which causes the count-rate asymmetries to change rapidly 
with source angle. As discussed in later chapters, another strong possibility is scattering from materials located near the detector, which would also vary erratically for sources at short distances. Otherwise, it appears that our 5-min measurements give reasonable results for the $\mathrm{PuO}_{2}$ sources at distances out to 8-9 $\mathrm{m}$, but 5-6 $\mathrm{m}$ is a more practical limit for the asymmetries and angles in the ${ }^{252} \mathrm{Cf}$ and gamma-ray cases.

Location Determination. It is difficult to visualize the position accuracies that result from the angle errors presented in Figs. $4.4 \mathrm{a}-\mathrm{c}$. An alternative presentation is shown in Figs. 4.5a-c, where we have marked the actual source locations by small symbols like those in Figs. 3.3a,b. The line segments attached to these symbols lead to the measured source locations obtained from the known distances and the experimentally determined angles. In this sense, the segments function as "error bars" on the source locations. In Fig. 4.5a the different symbols refer to data for different sources; in Figs. 4.5b,c they indicate data from different detector arrays. Not all measurements are included; as indicated in the captions, those with large statistical uncertainties have been omitted for clarity. Most of the remaining data give reasonable source locations, although there are certainly cases where the systematic error in the angle is very large despite having a small statistical uncertainty.

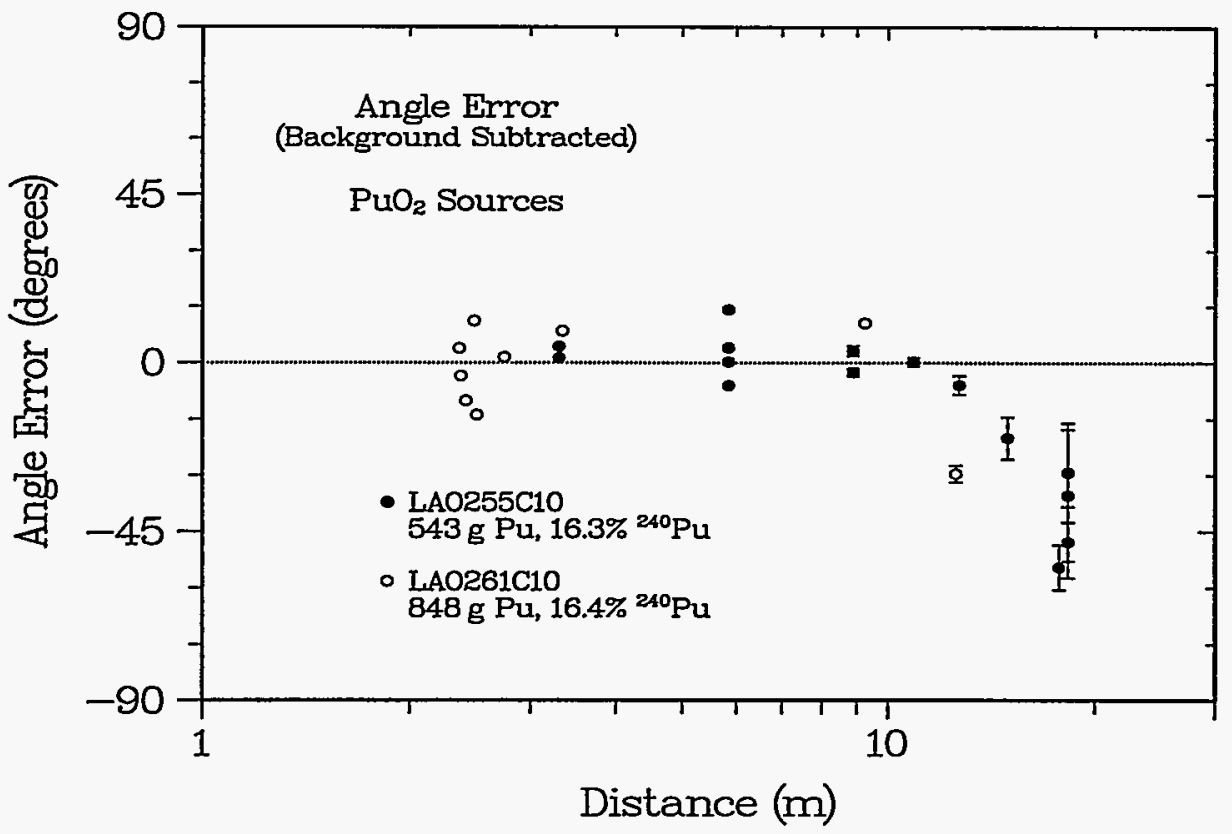

Fig. 4.4a. Distance-dependent error in the angle determinations for the $\mathrm{PuO}_{2}$ measurements shown in Fig. 4.3a.

Summary. The formalism for polarizations $P$ and analyzing powers $A$ developed in previous studies provides a useful method for interpreting the count-rate asymmetries $R$ observed with our segmented detectors. In both the angle-averaged data sets in Figs. 4.1a-c and in the individual measurements in Figs. 4.2 and 4.3, the cancellation of systematic errors provides much better results for the direction of the asymmetry than for its magnitude. Nevertheless, in our experiments the accuracy of the angle information is still limited mainly by systematic effects such as room scattering and shadowing between detector rods, not by counting statistics. For the sources used here, which represent typical neutron and gammaray outputs from a few kilograms of low-burnup.plutonium, there appears to be adequate 


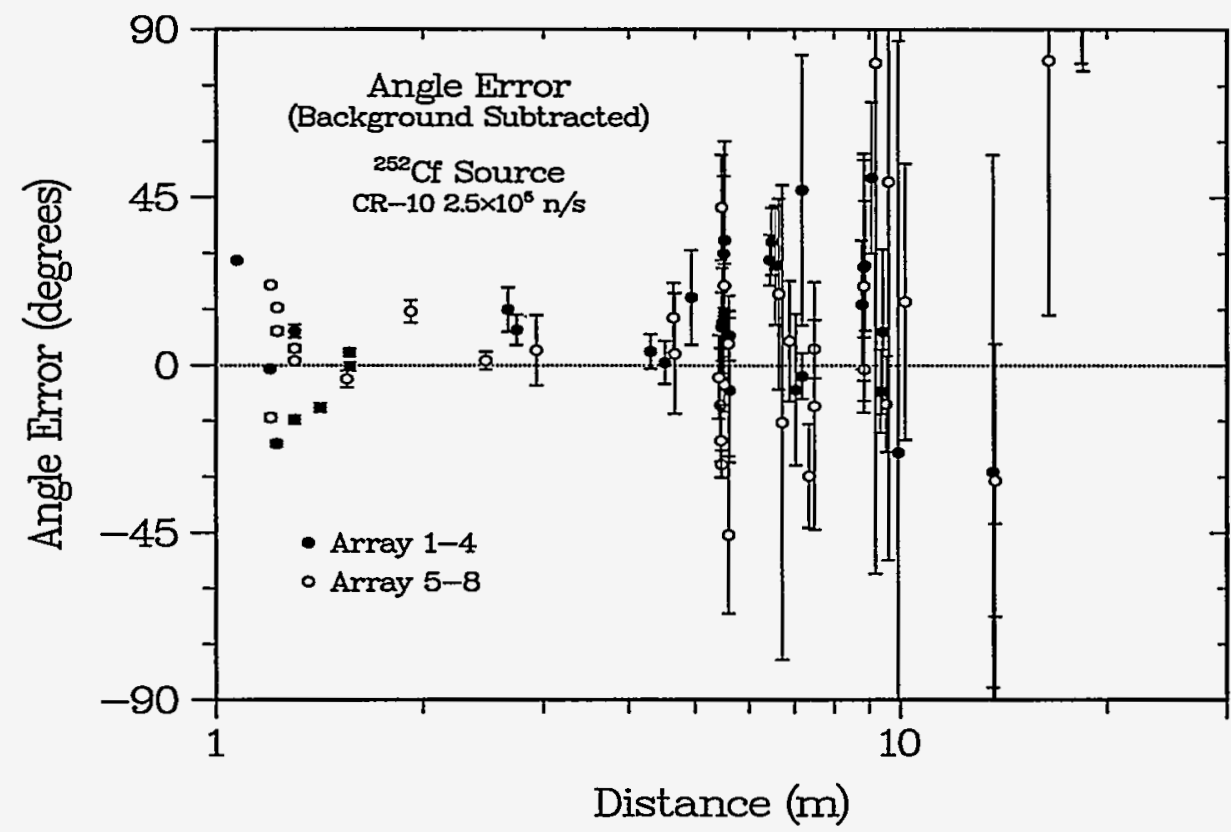

Fig. 4.4b. Distance-dependent error as in Fig. 4.4a, but for the ${ }^{252} \mathrm{Cf}$ source measurements shown in Fig. 4.3b.

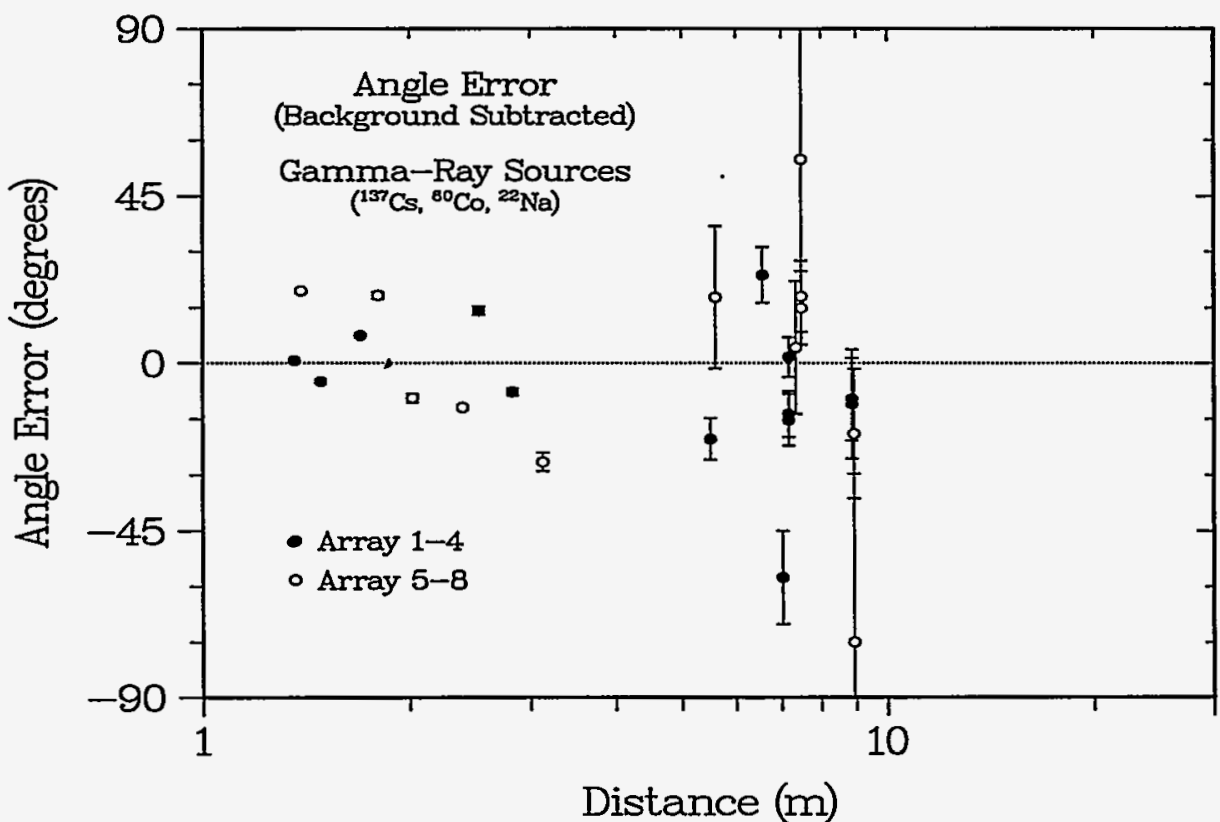

Fig. 4.4c. Angle errors as in Figs. 4.4a,b, but for the gamma-ray data shown in Fig. 4.3c.

sensitivity within counting times of $5 \mathrm{~min}$ to provide accuracies of about $\pm 10^{\circ}$ for source distances out to 5-8 m. Again, however, it is important to remember that the existing detectors are quite small, and greater range could be obtained using larger scintillators. 


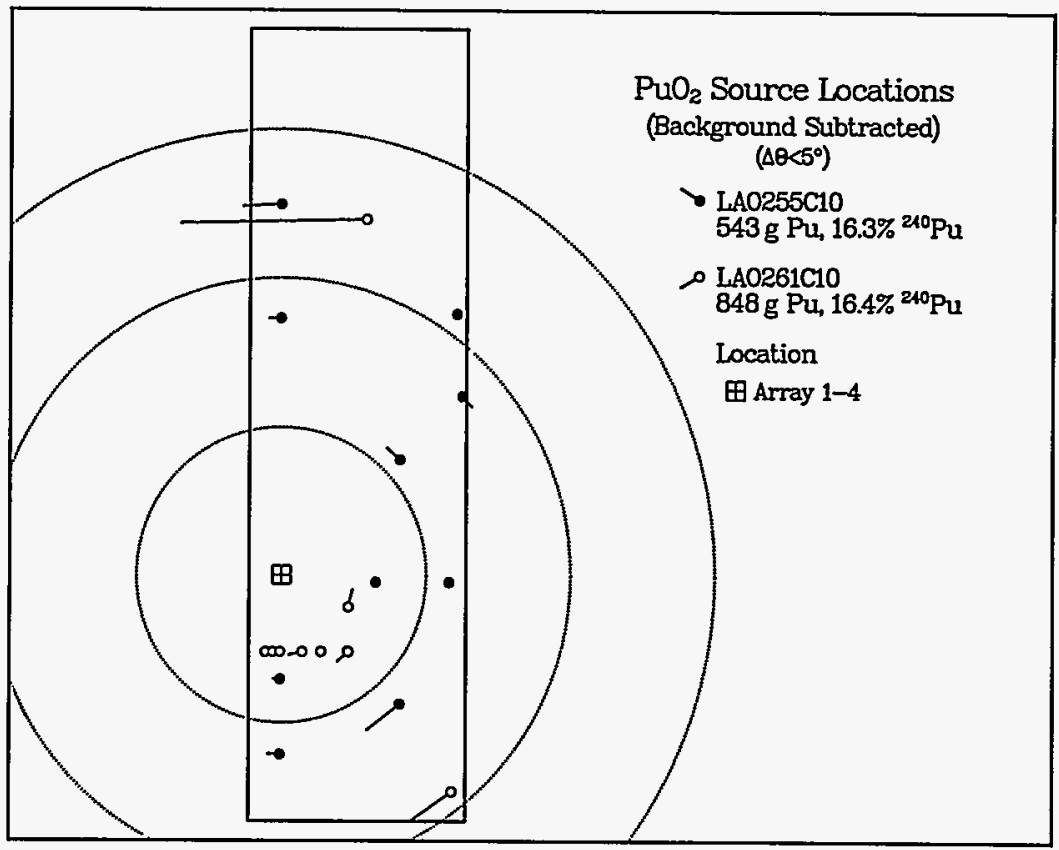

Fig. 4.5a. $\mathrm{PuO}_{2}$ source locations. The symbols show the actual source locations; the line segments point to the locations calculated using the measured angles and recorded distances. Measurements with angle uncertainties greater than $\pm 5^{\circ}$ are not shown. The dotted circles are at 5-m intervals.

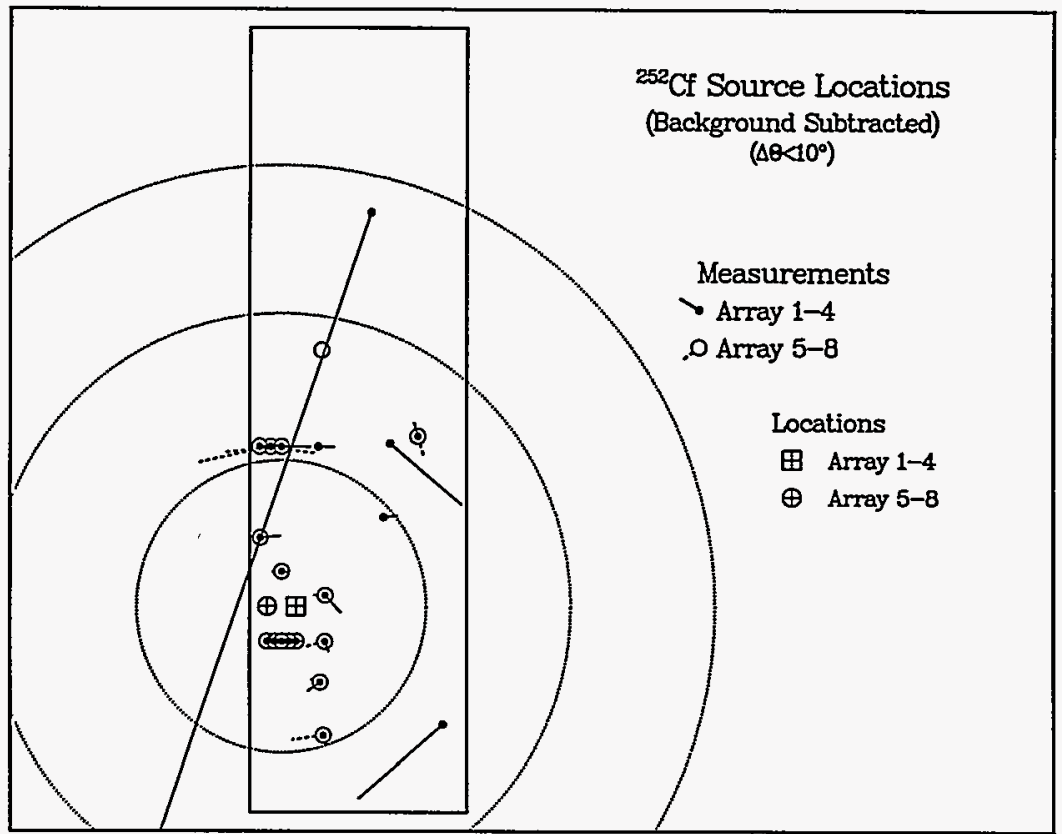

Fig. 4.5b. Source locations as in Fig. 4.5a, but for a ${ }^{252} \mathrm{Cf}$ source and omitting measurements with angular uncertainties greater than $\pm 10^{\circ}$.

\section{MOVING SOURCES}

Overview. The above measurements were intended to represent a search for nuclear materials, that is, using a moving detector to find a stationary source. Unfortunately, our 


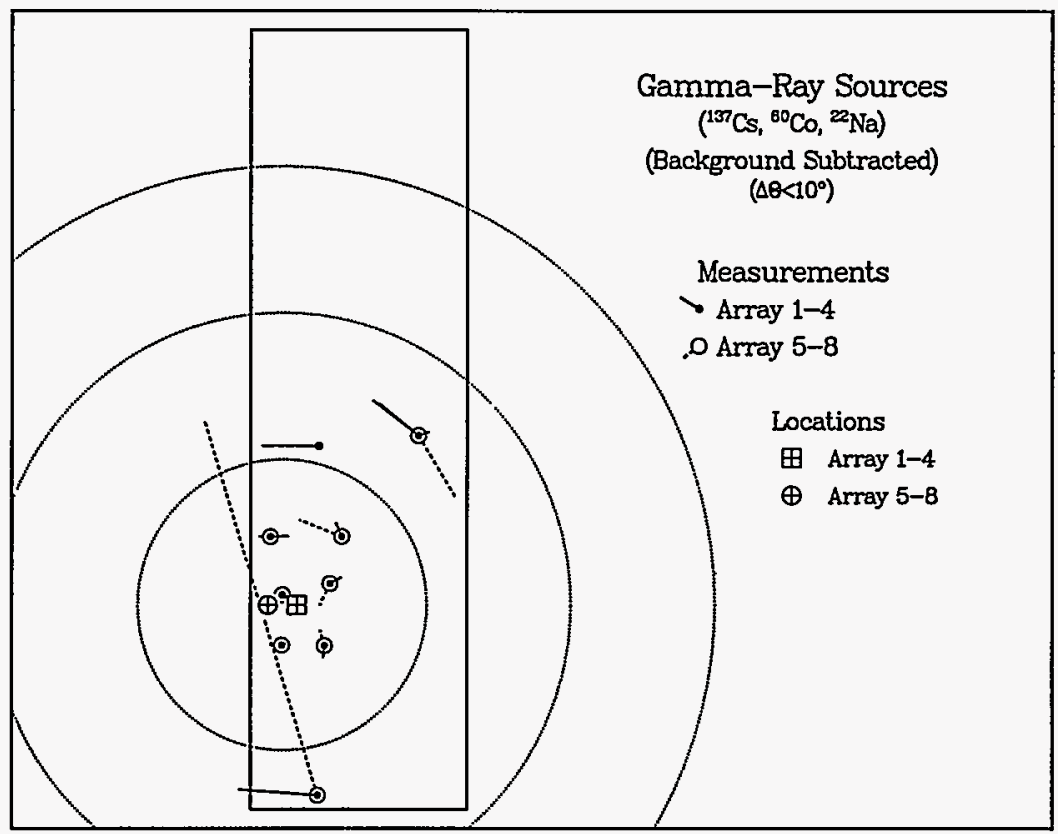

Fig. 4.5c. Source locations as in Fig. $4.5 \mathrm{~b}$, but for the set of three gammaray sources.

prototype detector arrays are not very portable, so the experiments were performed using fixed detectors and sources at different locations-and then the results were plotted versus separation distance to represent a search scenario. In this chapter we reverse this viewpoint and use a pair of stationary arrays to provide information about the location of a moving source. Although the layout, detectors, and analyses are not optimal, the results nevertheless indicate that directional detectors may be useful for applications such as vault monitoring.

Individual Count Rates. As shown in Fig. 5.1, for the moving-source measurements the two detector arrays were kept on the lab benches at the earlier center-to-center separation of $1 \mathrm{~m}$. Starting near the top of the figure, the CR-10 ${ }^{252} \mathrm{Cf}$ source was moved in 1-m steps along the path shown by the dotted line, stopping at each of the indicated points. Two different runs were made: a "fast-moving" pass with 1-min accumulations at each point, and a "slow-moving" run with longer 5-min accumulations. For the latter run, the backgroundcorrected count rates for the individual rods of the two arrays are shown in Figs. 5.2a,b. At the outset, the count rates differ only because of statistics and scattering effects; midway through the run, however, the rates clearly diverge. Just as expected from Fig. 5.1, the rates for the two rightmost rods in each array $(3,4$ and 7,8$)$ first increase and then decrease, followed by an increase for the lower two pairs of rods $(2,4$ and 6,8$)$. These figures emphasize an important feature of using a directional detector as a vault monitor: different types of information are combined in a unique fashion, which provides both measurement redundancy and security against outside interference.

Field Directions. In Fig. $\mathbf{5 . 3}$ we show the analysis results for the runs with the slowmoving (left) and fast-moving (right) sources. The small circles are again the actual source locations, and the solid and dashed vectors are the results for arrays 1-4 and 5-8, with array 5-8 located nearer to the wall. Each vector's direction indicates the calculated angle between the detector and the source; that is, the vector should point toward the source 


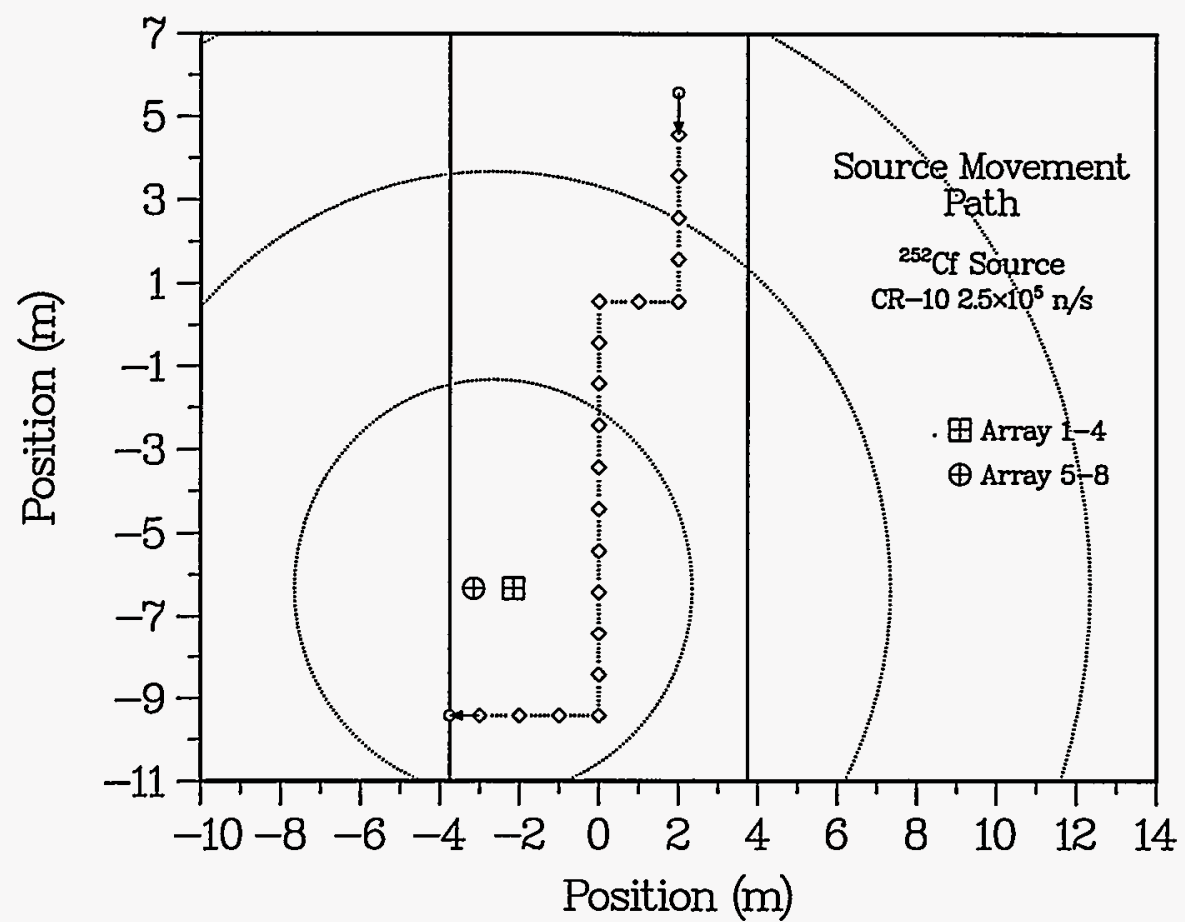

Fig. 5.1. Path followed by the slow- and fast-moving ${ }^{252} \mathrm{Cf}$ sources in the moving-source tests in the CMR training area. The distance between the points is $1 \mathrm{~m}$.

and away from the corresponding detector. The lengths of the vectors simply indicate the magnitudes of the count rates. Although the directions and magnitudes are generally as expected, close inspection suggests a consistent bias that may indicate scattering from the nearby wall. Thus, our initial results indicate that obtaining approximate source directions is quite possible, but systematic problems may limit the ultimate accuracy; these problems will be examined in greater depth in the next chapter.

Distance Estimates. An obvious problem with the position determinations thus far is the limitation of finding the direction to the source but not the distance. This issue may not be so serious in searching for a stationary source with a portable detector, but it is very important for tracking the movement of sources in a storage vault. Because room scattering causes the measured polarization to decrease with source separation, an approximate distance could be obtained using the asymmetry measured without background subtraction. Unfortunately, Fig. 4.2a indicates that the distance correlation is often small compared with other effects. A better candidate is the $k / r^{n}$ correlation shown in Figs. 2.4a-c between separation distance and count rate, which shows much less fluctuation. Inverting this relation requires determining the $k$ and $n$ parameters; to this end, Fig. 5.4 shows the observed count-rate dependences for the CR-10 source, either moving (present data) or stationary (from Fig. 2.4b). There appears to be a change in slope at the shortest distances, perhaps because of scattering from the heavy lab bench supporting the two detectors; nevertheless, we will use the single exponent $n=1.33$ in order to simplify the inversion. To determine the value of $k$ that normalizes the distance to the count rate, we will use pairs of intersecting source vectors in the data set, which provides an independent estimate of the absolute distance scale. Knowing $k$ and $n$ allows the count rate for each measurement to be converted into an estimated distance, which can then be combined with the measured angle to give the source location. In fact, the major reason for employing two arrays (1-4 and 5-8) was 


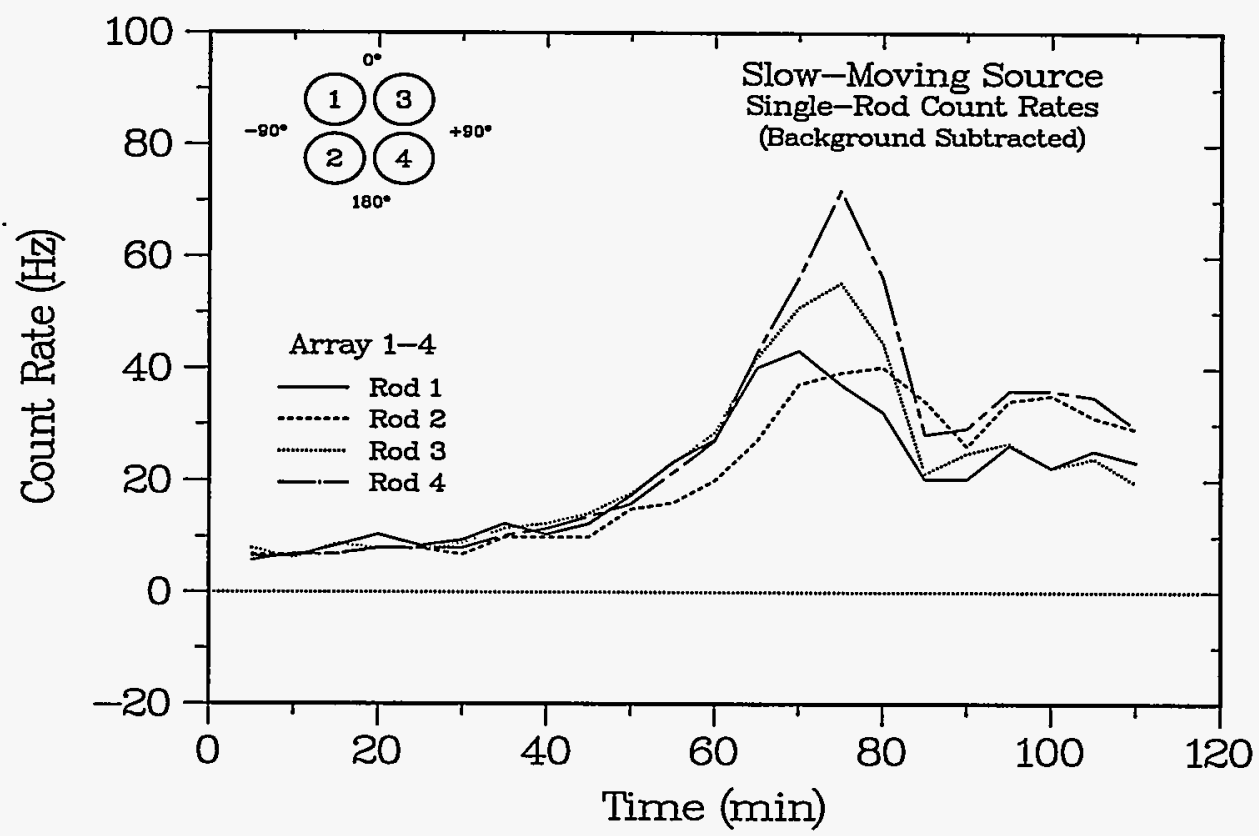

Fig. 5.2a. Individual-rod count rates observed for array $1-4$ with a ${ }^{252} \mathrm{Cf}$ source stepping along the path in Fig. 5.1 at the rate of $1 \mathrm{~m}$ per $5 \mathrm{~min}$.

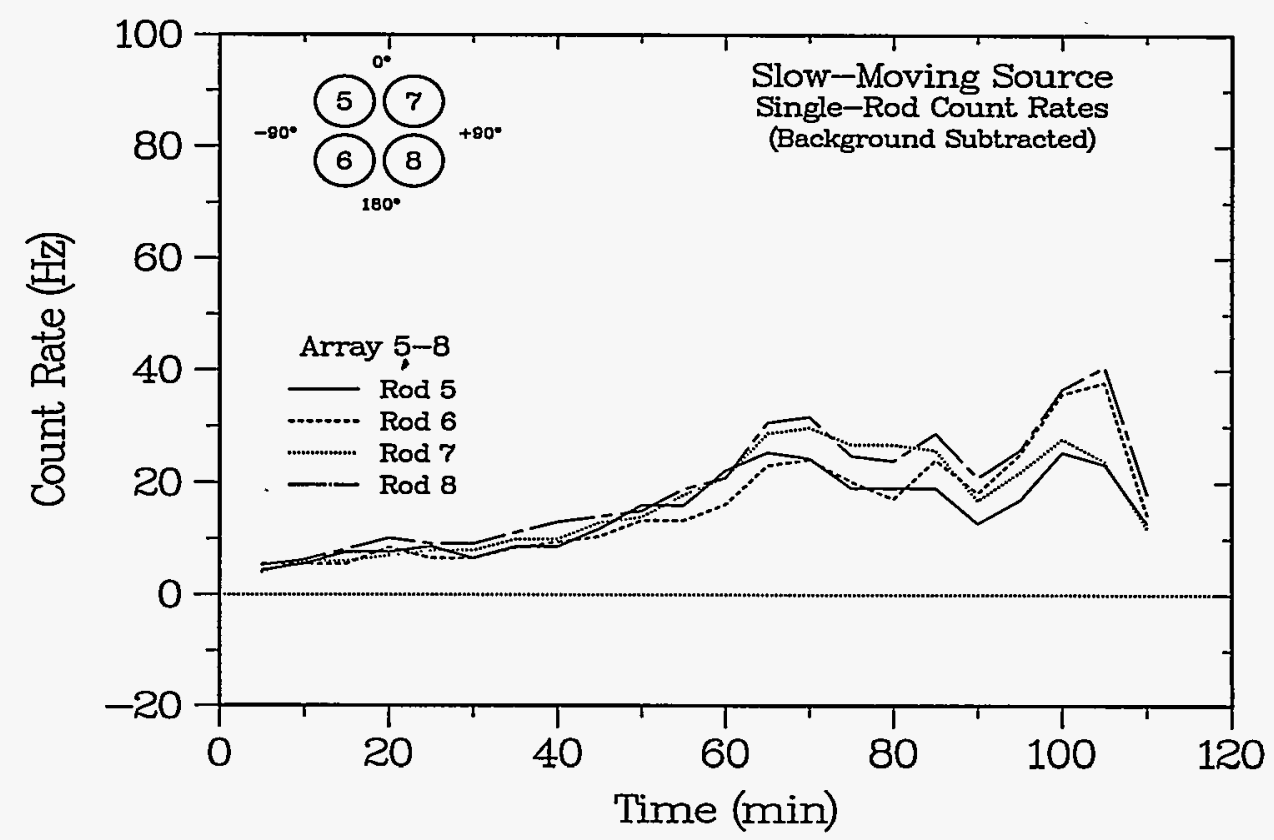

Fig. 5.2b. Individual-rod count rates as in Fig. 5.2a, but for the moredistant array 5-8.

to study this concept of a "radiation rangefinder," which uses multiple angle measurements to locate a source. The next several figures illustrate this approach.

Rangefinder Results. The first step in the procedure calibrates the distance scale by using the available intersections between the source vectors from the two arrays. As 


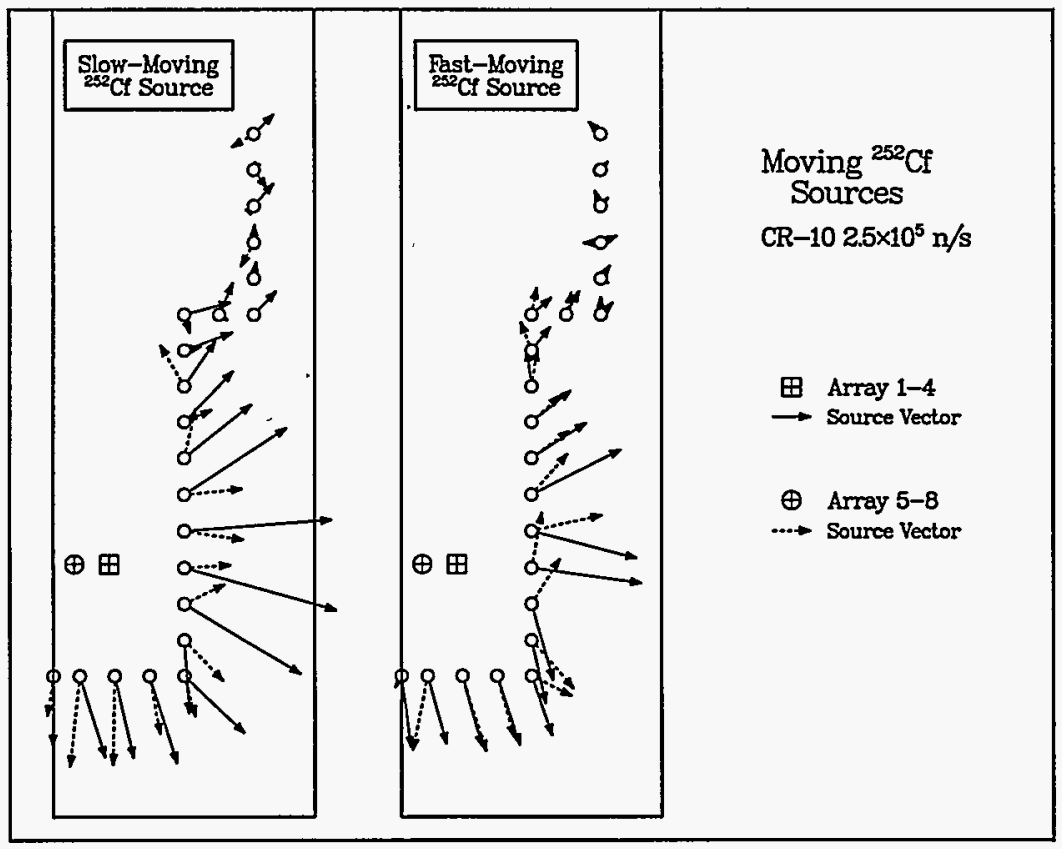

Fig. 5.3. Display of calculated direction vectors for a ${ }^{252} \mathrm{Cf}$ source stepping along the predetermined path shown in Fig. 5.1. The left panel uses a slow rate of $1 \mathrm{~m}$ per $5 \mathrm{~min}$; the right panel uses a faster rate of $1 \mathrm{~m}$ per $1 \mathrm{~min}$.

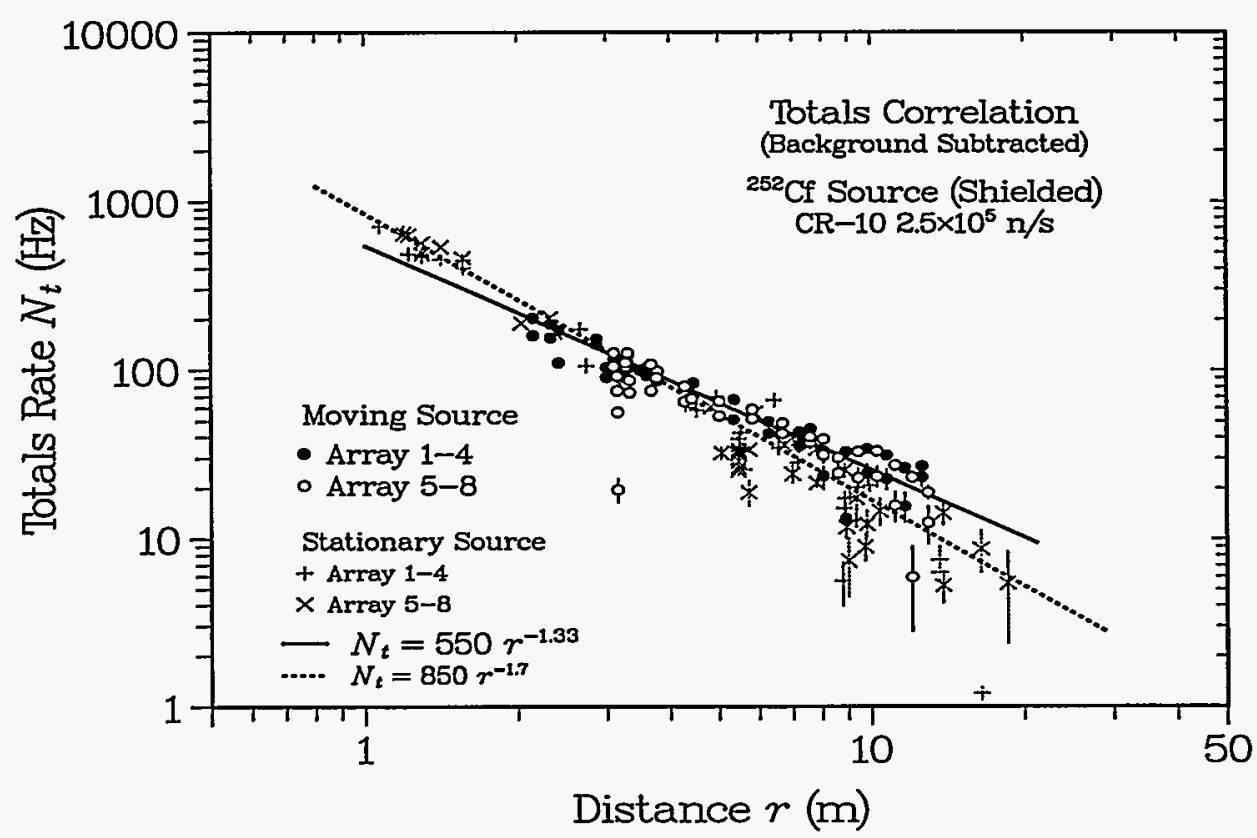

Fig. 5.4. Count-rate dependences for several ${ }^{252} \mathrm{Cf}$ source scans, showing different possible $k / r^{n}$ variations.

shown in Fig. 5.5a, relatively few intersections exist in the present data set, and some of these may be accidental, especially where the vectors converge near the detectors. To 


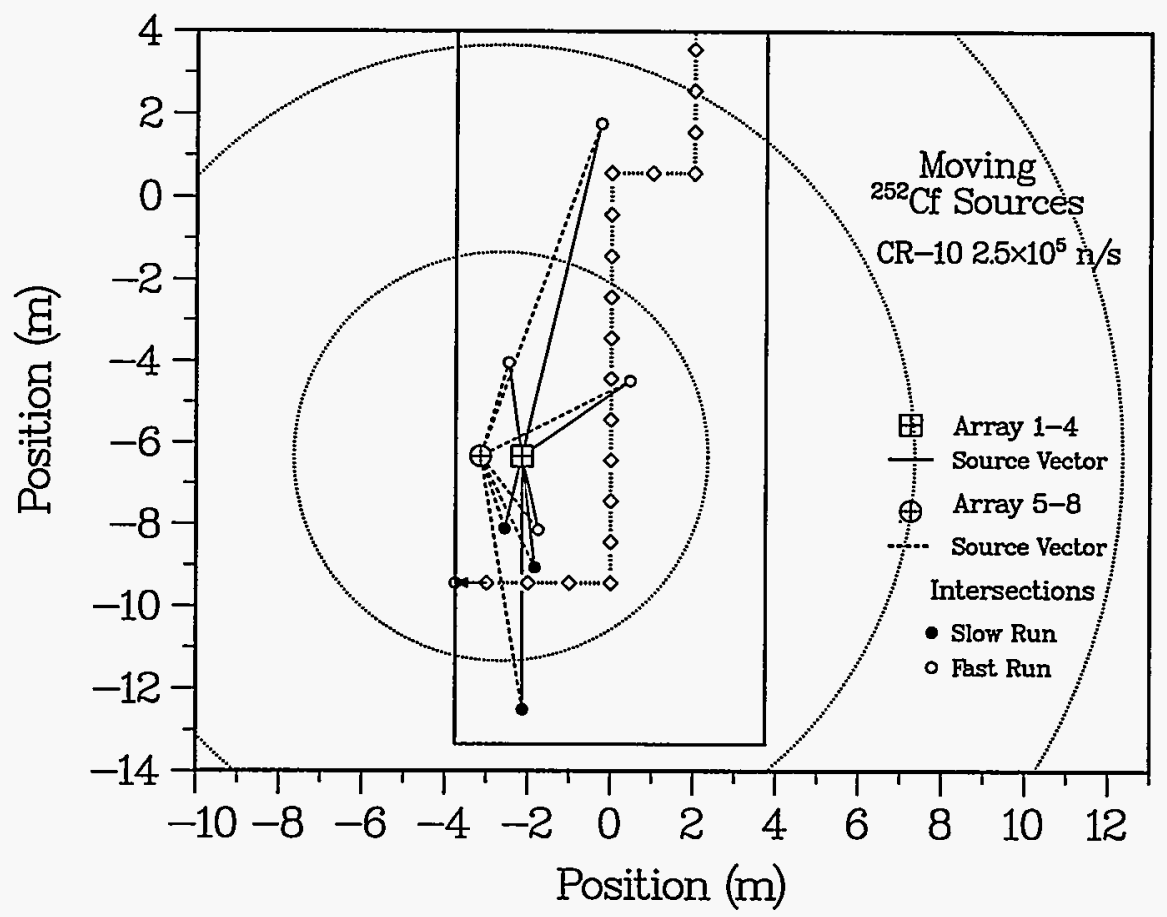

Fig. 5.5a. Intersection points for source vectors from the present runs with slow- and fast-moving ${ }^{252} \mathrm{Cf}$ sources. Only seven intersections were obtained from a total of 44 measurements.

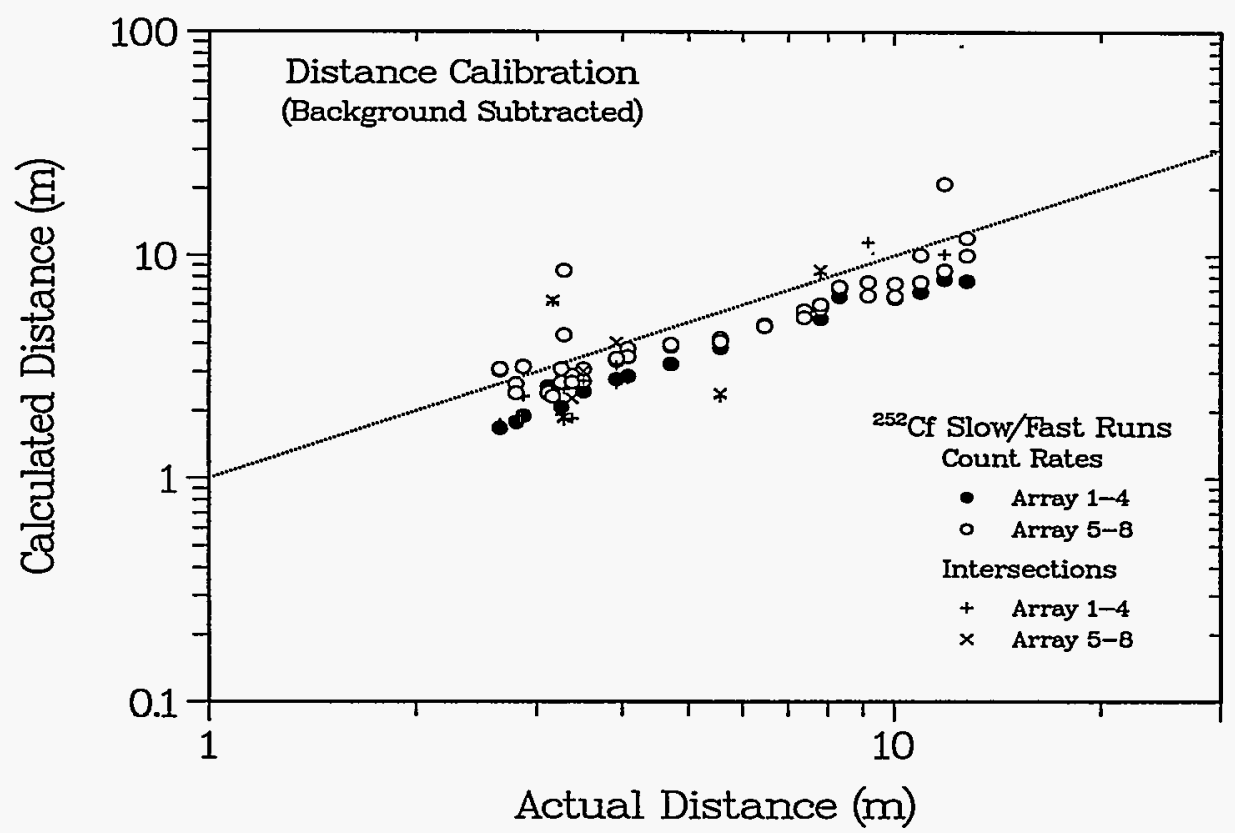

Fig. 5.5b. Actual and calculated distances, determined both from the vector intersections in Fig. 5.5a and from the count-rate dependence in Fig. 5.4 as normalized to the intersection points.

compensate, we have deliberately excluded any intersection located within $50 \mathrm{~cm}$ of either detector. Also, because the location accuracy is better for vectors that cross at right angles, we have weighted each $k$-value by the sine of the angle difference, $\sin \left(\theta_{58}-\theta_{14}\right)$. The results 
of the distance determination are given in Fig. 5.5b. The original 14 intersection distances are shown by the + and $\times$ symbols, and the complete set of rate-derived averages are shown by the solid and open circles. Unfortunately, because the convergence near the detectors strongly biases the average toward small separations, the corrected distances systematically underestimate the true values by about $30 \%$. We have therefore adjusted our final values by this factor, which gives the tracks shown in Fig. 5.6. Each array provides a separate set of locations for each run, but the array values have been combined in order to compare the slow and fast runs. Only the last dozen points are shown, because the angle uncertainties at the greater distances are very large. Given that the results from the two runs are very similar, the accuracy appears to be dominated by systematic effects and not by counting statistics.

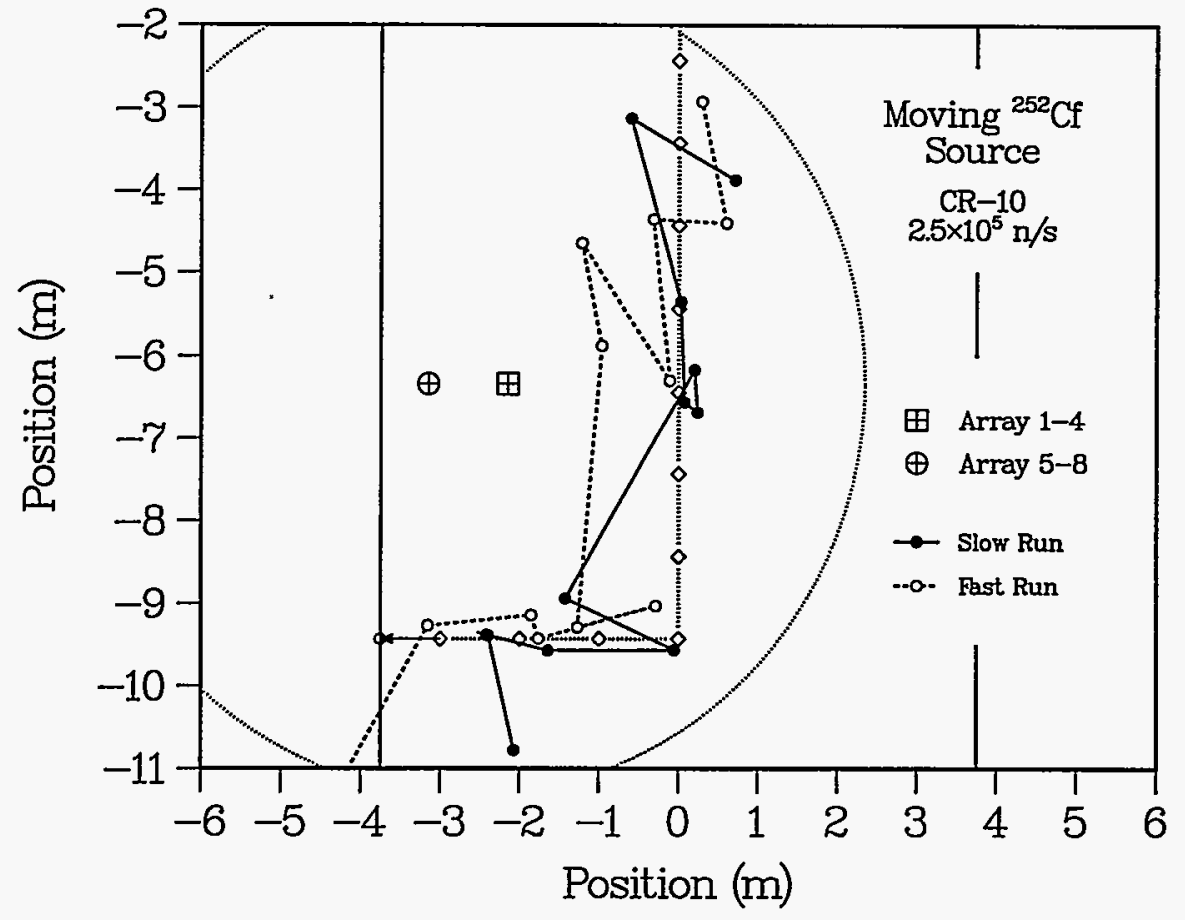

Fig. 5.6. Corrected locations for slow- and fast-moving ${ }^{252} \mathrm{Cf}$ sources. Distances are determined using the $k / r^{n}$ count-rate dependence, as normalized to the vector intersections but multiplied by 1.3. (Only the last 12 measurements in each scan are shown.)

Summary. This chapter has laid out the basic approach for detecting and tracking a moving source using stationary directional detectors. Although the present changes in source position were made very slowly, more rapid movements could be monitored by using larger detectors. As with conventional detectors, count-rate changes clearly indicate source movements, but correlating the changes from multiple counters provides valuable redundancy, even if the counters are not part of a segmented array. The more ambitious program initiated here, where a pair a directional arrays is used to construct a radiation rangefinder, reveals several complex problems, particularly in using vector intersections to calibrate the parameters in the $k / r^{n}$ relation between count rates and source distances. Unfortunately, the limited base-line separation and counting statistics for the present measurements make it difficult to evaluate the ultimate utility of the technique. In the next chapter, improvements in the detector geometry and source intensity lead to more definitive results. 


\section{TA-35 MEASUREMENTS}

Overview. Although the results of the CMR measurements in the previous chapters are promising, to provide a more complete evaluation we obtained additional data using the NIS-5 facilities located at TA-35. These measurements differ from those described above in three main ways: (1) the source strength was increased by about a factor of 3 to $6.8 \times 10^{5} \mathrm{n} / \mathrm{s}$ by using a larger ${ }^{252} \mathrm{Cf}$ source (CR-11); (2) the base-line separation between the two arrays was increased to $2.87 \mathrm{~m}$, essentially the maximum possible with the existing hardware; and (3) the data were taken in a roughly cubical room $8.5 \mathrm{~m}$ on a side, which has massive shielding walls but contributes less floor and ceiling scattering. The measurements comprise three data sets: Sec. 6.1 describes lengthy stationary-source accumulations that address the limitations of counting statistics; Sec. 6.2 discusses moving-source measurements made along an 8-m track; and Sec. 6.3 describes a "realistic" measurement made using stationary sources with largely unknown characteristics. The first of these sections develops the analysis techniques, and the remaining two illustrate the analyses for two different situations.

\subsection{Stationary Sources}

Layout. The accuracy of the rangefinder results in the previous chapter appears to be limited by a combination of statistical precision and systematic effects such as detector geometry and room scattering. To investigate the statistical problem, a set of measurements was made using the more intense CR-11 source and a series of 5-min accumulations that totaled 2-12 hours at each position. To emphasize the systematic problems, the source positions were arranged to cover almost the entire room. The analysis of these measurements follows the rangefinder approach of the previous chapter, relying on a set of vector intersections to calibrate a $k / r^{n}$ estimate of the source distances.

Analysis and Results. As in Chap. 5, the first step uses the vector intersections to provide an initial set of source distances. In Fig. 6.1 we show the eight source positions as large dotted circles with $0.5-\mathrm{m}$ radii that provide a scale. The open circles are the intersection locations from a long background run; as expected, their distribution is nonuniform and clusters near the detectors. This distortion is even more severe for the source points (closed circles), whose positions do not appear to be closely related to the source locations. Figure 6.2a continues the analysis, showing the very good correlation between total count rates and source distances as well as between the CMR and TA-35 measurements; interestingly, the same $1 / r^{1.33}$ dependence describes both data sets reasonably well. Figure $6.2 \mathrm{~b}$ shows the distances determined from the intersections and the $k / r^{n}$ dependences, with the average weighted as before by $\sin \left(\theta_{58}-\theta_{14}\right)$. In contrast to the CMR results in Fig. $5.5 \mathrm{~b}$, no additional renormalization is needed with the longer base line of the TA-35 measurements. Finally, Fig. 6.3 shows the resulting source locations. The position accuracy is excellent in the most favorable cases, such as at points located away from walls or on the axis between the detectors at distances within a few meters. Otherwise, there can be large errors. For sources at distances over $5 \mathrm{~m}$, the statistical uncertainty on the angle determination for each 5-min accumulation is large enough to create a broad arc of location values, although the centroid is essentially correct. However, for detectors located near both a wall and the detector, a small statistical uncertainty is combined with a large systematic error, which gives an unexpected result: the source is well localized, but at the wrong location. This behavior indicates that room scattering can greatly affect the radiation field as seen at the detector, an entirely reasonable result.

\subsection{Moving Sources}

Layout. Based on the results in the previous section, we have also repeated the experiments made at CMR with moving sources, but now using the stronger CR-11 source and 


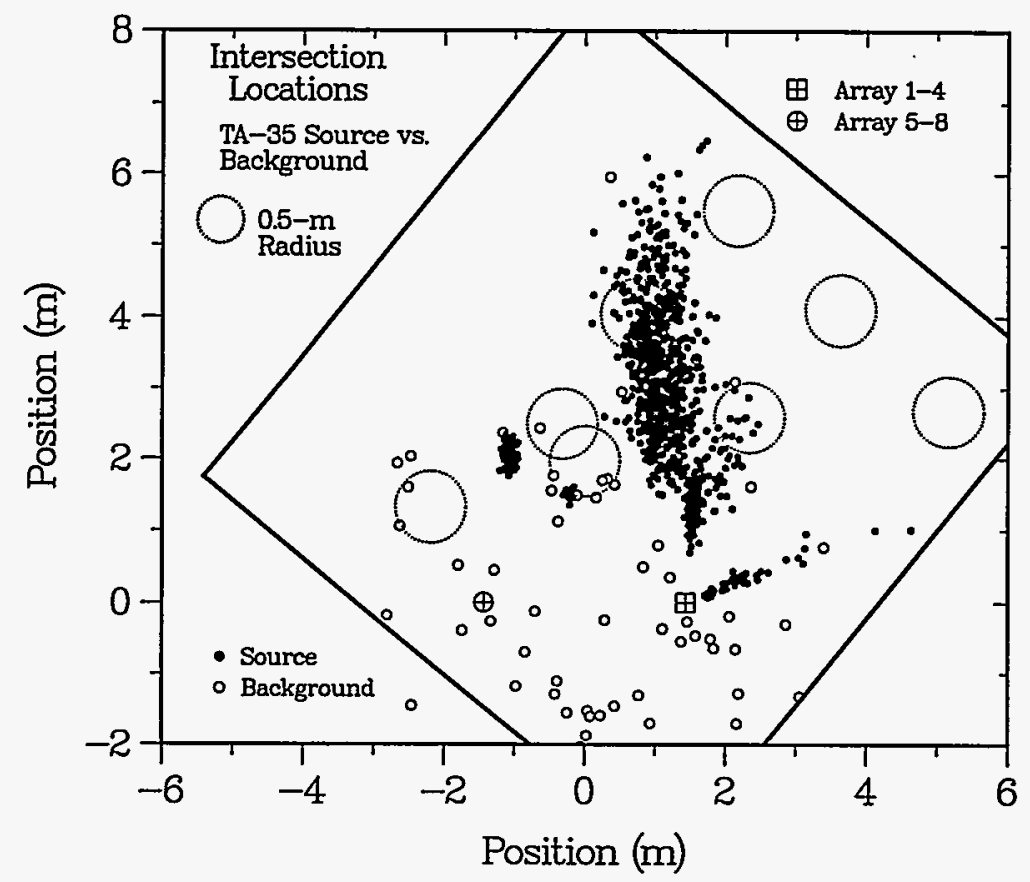

Fig. 6.1. Layout of the experiments at TA-35. The heavy lines show the massive shielding walls of the $8.5 \times 8.5 \mathrm{~m}^{2}$ target room, and source locations are shown by dotted circles. Closed circles show intersections for source measurements; open circles are background intersections.

longer base line between the rangefinder arrays. The source movements were again in 1-m steps with accumulation times of $5 \mathrm{~min}$ at each position.

Analysis and Results. As before, the analysis first determines the vector intersections and then uses these locations to calibrate the $k / r^{n}$ dependence. Figure 6.4a shows the source path and two sets of intersections, one for each of two measurement passes. The intersections appear to provide reasonable values for the source positions, as confirmed by the agreement shown in Fig. 6.4b between the actual distances and the intersection values. Given that the values for the $k / r^{n}$ dependences are well behaved, the errors in the source locations in Fig. 6.5 are mainly caused by problems with the angle values. As before, the analysis for each run gives two sets of positions; we have averaged the values from the two runs to give the two paths shown, one for each array. Both results behave poorly near the start of the track, where the separations of 6-8 $\mathrm{m}$ limit the statistical accuracy. A different situation exists near the end of the track, where the source approaches the massive shielding wall. For array 5-8, the direct fluence from the nearby source dominates the wall scattering, so the calculated angle is correct. For the more distant array 1-4, which is located well away from the source, scattering from the wall dominates, so the observed angle is significantly in error. These conclusions are supported by the results of the asymmetry model described in Chap. 4. Just as expected, without background subtraction the measured asymmetries for both arrays increase as the distances decrease at the start of the track. At the end of the track, however, the values for array 5-8 begin to drop despite the decreasing distance, while those for array 1-4 continue to rise despite the increasing distance. This behavior can only be understood by comparing the fluences that reach the detectors directly from the source versus those that scatter diffusely from the room or reflect from the wall. As in the previous section, the detector responds strongly to a directional source of radiation, even if this source is only a reflected image. 


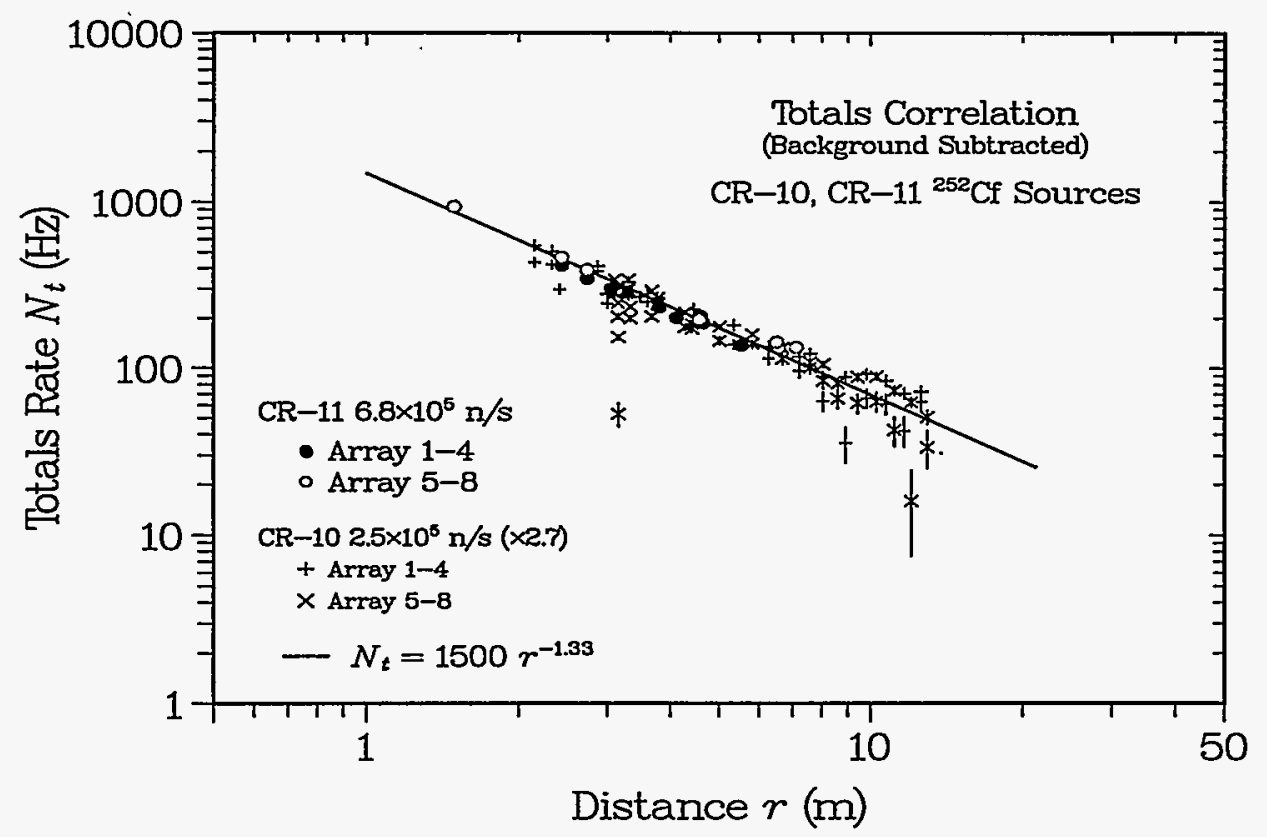

Fig. 6.2a. Total count rates versus distance for measurements with the CR-10 and CR-11 ${ }^{252} \mathrm{Cf}$ sources. The measurements at TA-35 (CR-11) and CMR (CR-10) both agree with an $1 / r^{1.33}$ dependence on distance.

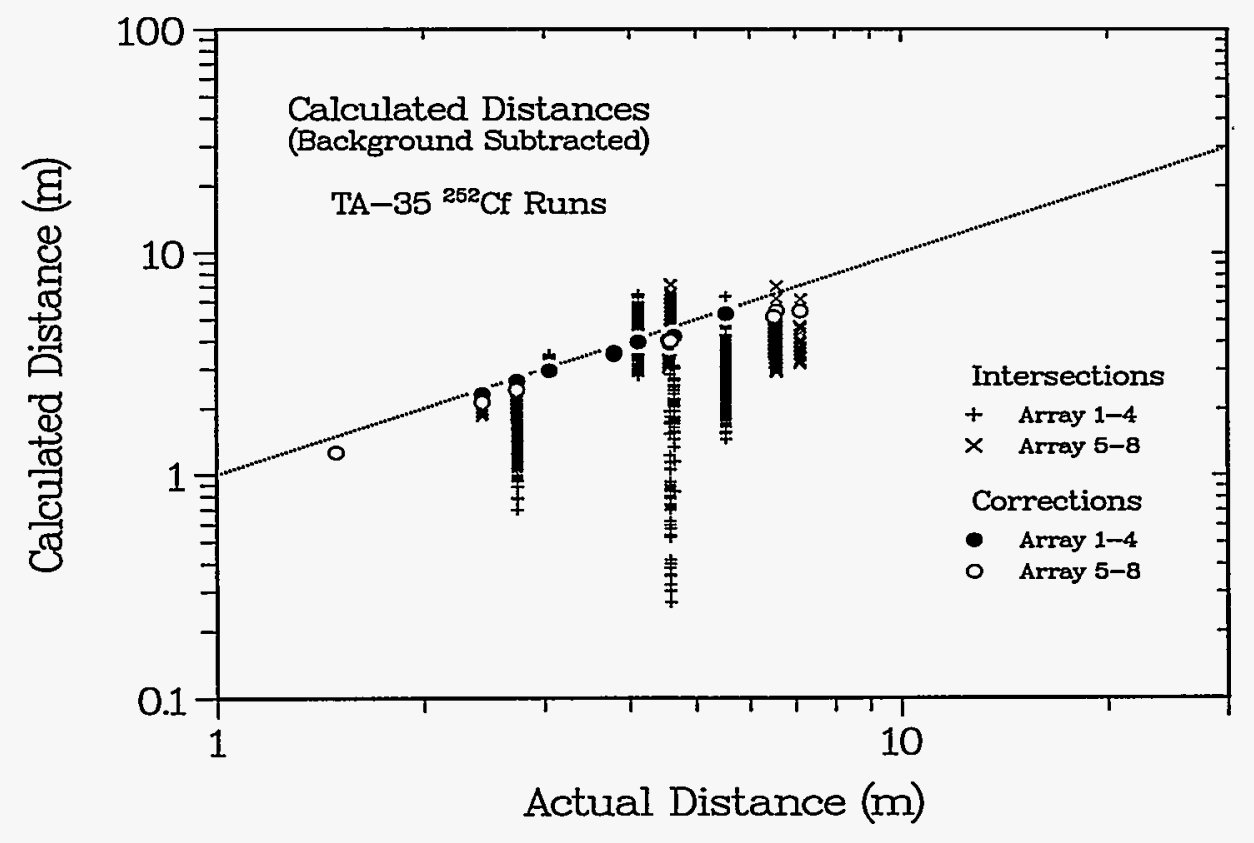

Fig. 6.2b. Distance determinations; the intersection values are shown as + and $\times$ symbols, and the normalized $k / r^{n}$ results are shown as closed and open circles.

TA-35 Stationary- and Moving-Source Summary. With the better statistics and longer base line used for the measurements in Secs. 6.1 and 6.2, it now appears that an important practical limit on the accuracy of the source location is often the effect of room 


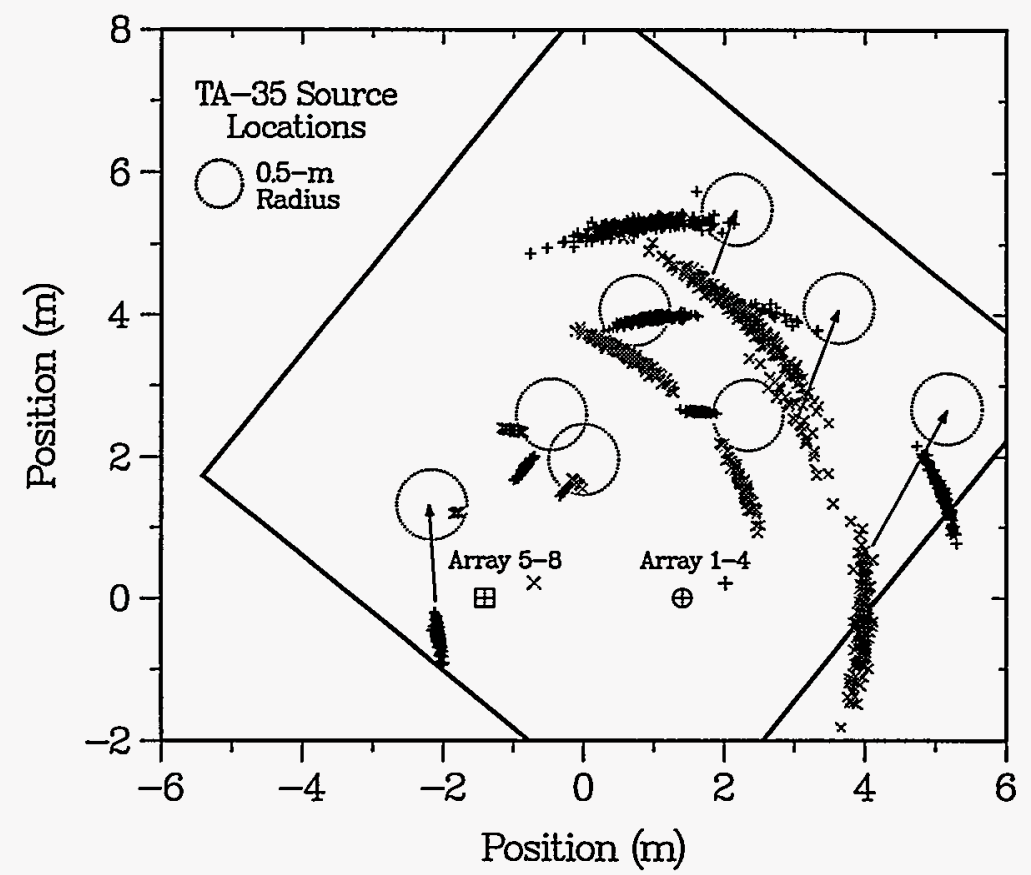

Fig. 6.3. Final locations obtained from the detector angles and the intersection-normalized distances. For nearby sources with no obvious scattering complications, the results are excellent. More distant sources or those located near walls show pronounced statistical or scattering effects.

scattering. Regardless of whether the source is moving or stationary, the radiation fluence at a remote detector location is inevitably a combination of direct and scattered fluences, and the detector merely measures the net local result-which may well point in a surprising direction, as we have seen.

\subsection{Unknown Source}

Problem Statement. Our final test replaces the CR-11 ${ }^{252} \mathrm{Cf}$ source used in the previous sections with an unknown, variable source. Over a week-long period, tests were carried out on a neutron coincidence counter placed on the opposite side of the room from our two arrays. Various combinations of ${ }^{252} \mathrm{Cf}, \mathrm{PuO}_{2}$, and $\mathrm{Am}-\mathrm{Li}$ sources were repeatedly cycled through the well-moderated counter, with the entire sequence preceded and followed by long background runs. This section describes the results of the data analysis for array 1-4, which was positioned $4.7 \mathrm{~m}$ away with a clear view of the source changes.

Count Rates. Figure 6.6a shows the total count rate during the time around the source measurements. The average background rates determined from the earlier and later measurements have already been subtracted; the residual rates at the beginning and end of the present range are consistent with zero. There appear to be four distinct sections of source measurements; these are separated by three short intervals where the count rates drop but do not reach zero. Inspection of the logs confirms this sequence of source tests. Because the tests use the ${ }^{252} \mathrm{Cf}$ and Am- $\mathrm{Li}$ sources in different combinations, the observed rates and the underlying energy distribution both change over time.

Asymmetry and Angle Analyses. During the background measurements before and after the source tests, the asymmetries recorded by the array fluctuated about zero with large uncertainties. As shown in Fig. 6.6b, once the sources were introduced, the asymmetries 


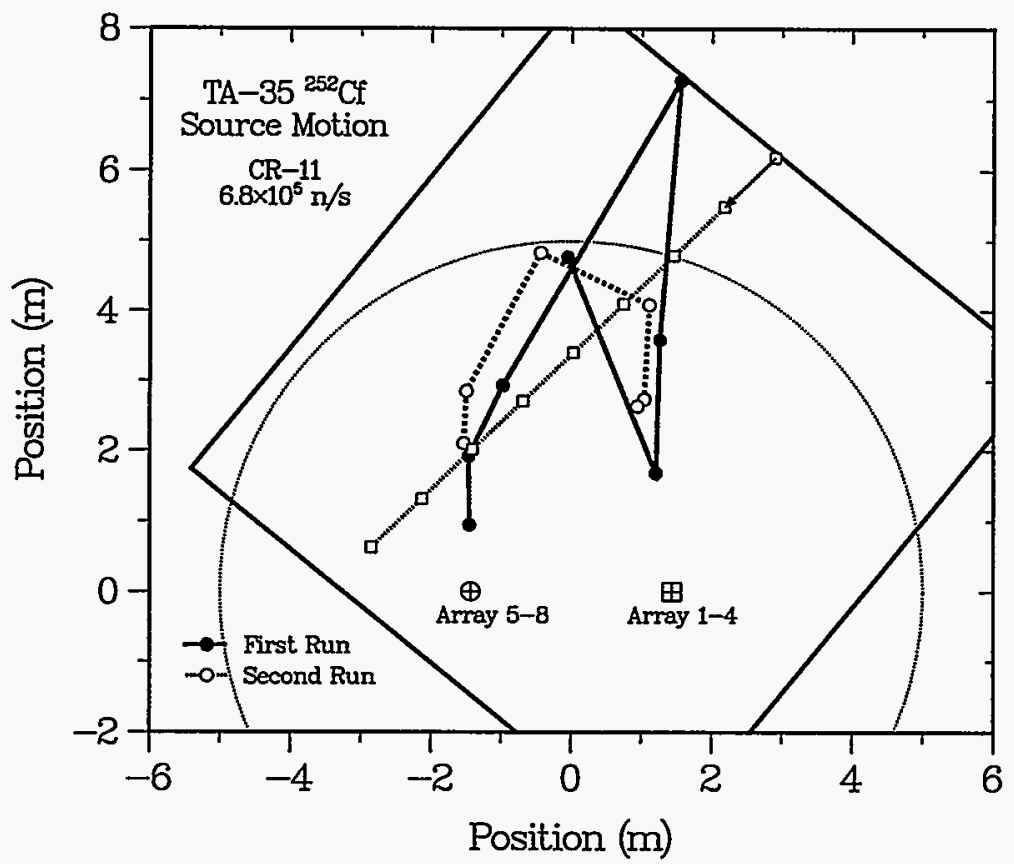

Fig. 6.4a. Moving-source locations determined from the intersections of the vectors from the two detector arrays. The open rectangles and straight dotted line line show the actual source path, and the solid and dashed lines connect the intersection points for two different data runs.

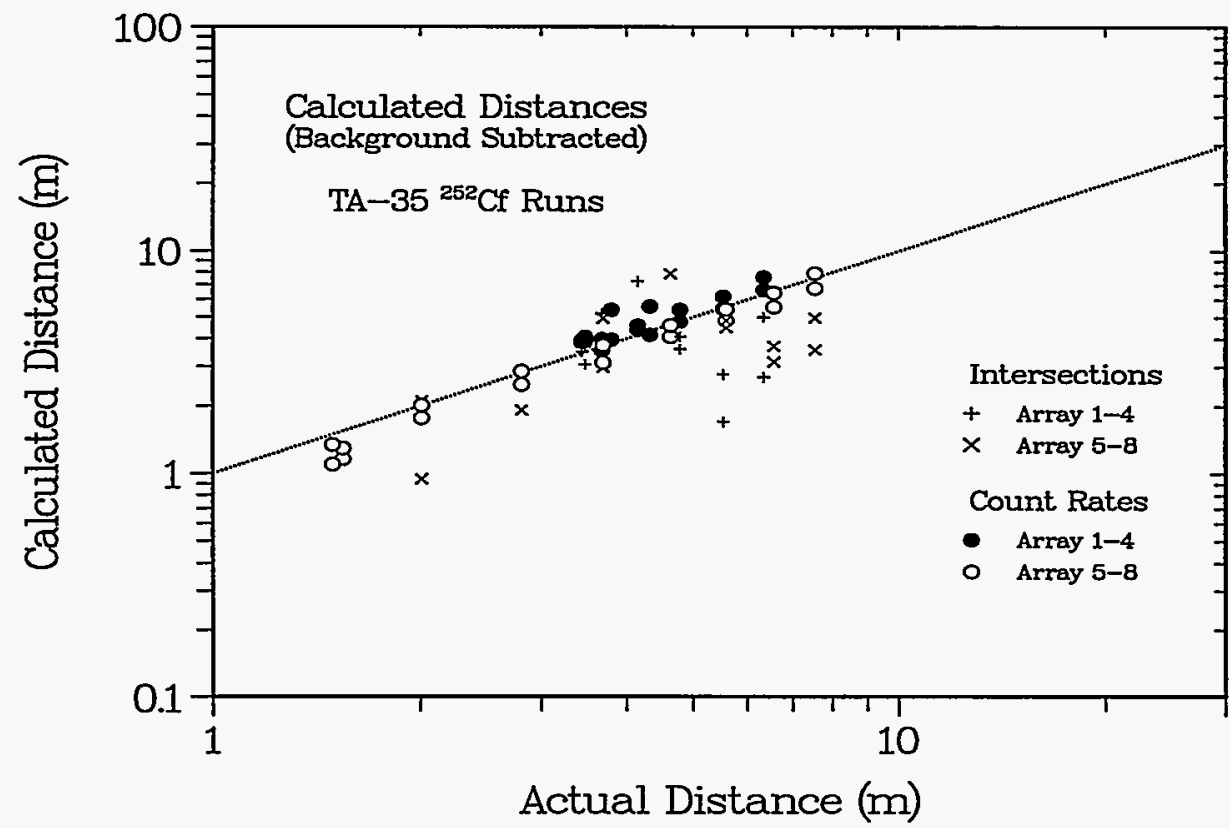

Fig. 6.4b. Corrected source-to-detector distances obtained by normalizing the $k / r^{n}$ dependences using the intersection points from Fig. 6.4a.

jumped up to $20-30 \%$ and remained clearly nonzero except during the source changes. The larger value for the last set of measurements suggests an increase in the detector's analyzing power, perhaps because of the lower average energy and hence greater attenuation for the 


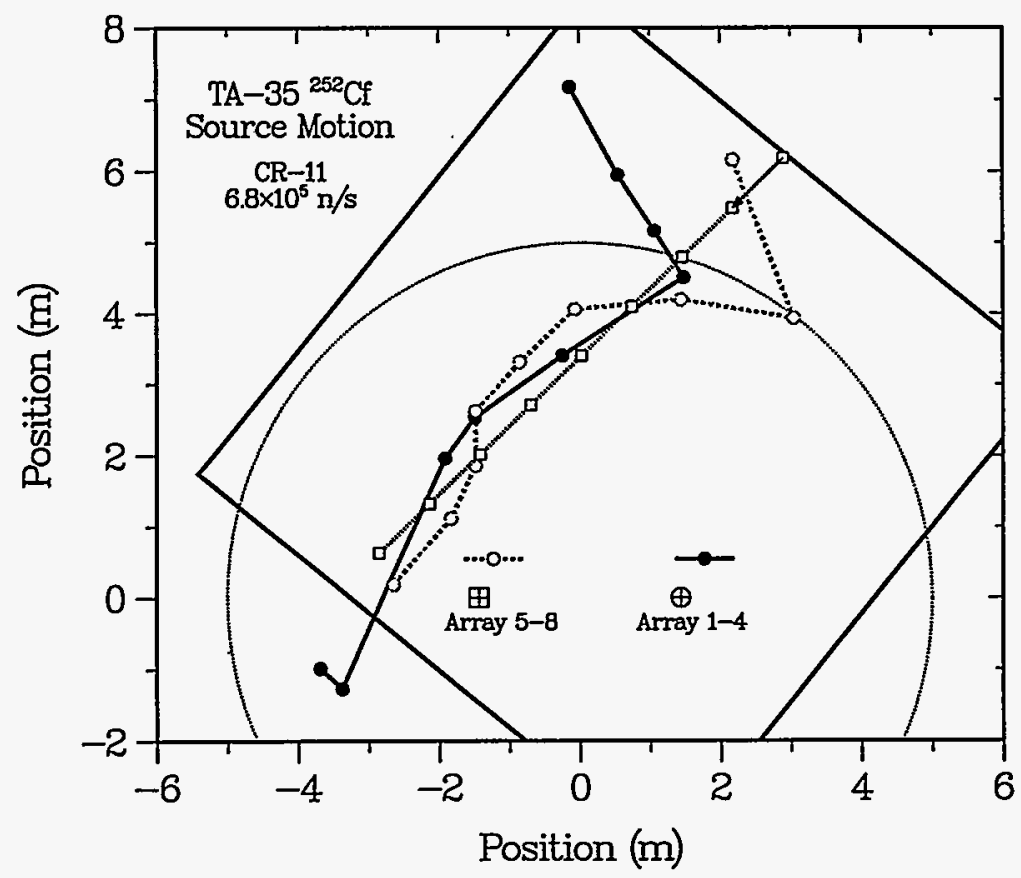

Fig. 6.5. Corrected source locations obtained by combining the array direction vectors with the $k / r^{n}$ distances. The values for the two arrays were obtained by averaging the results from two different runs.

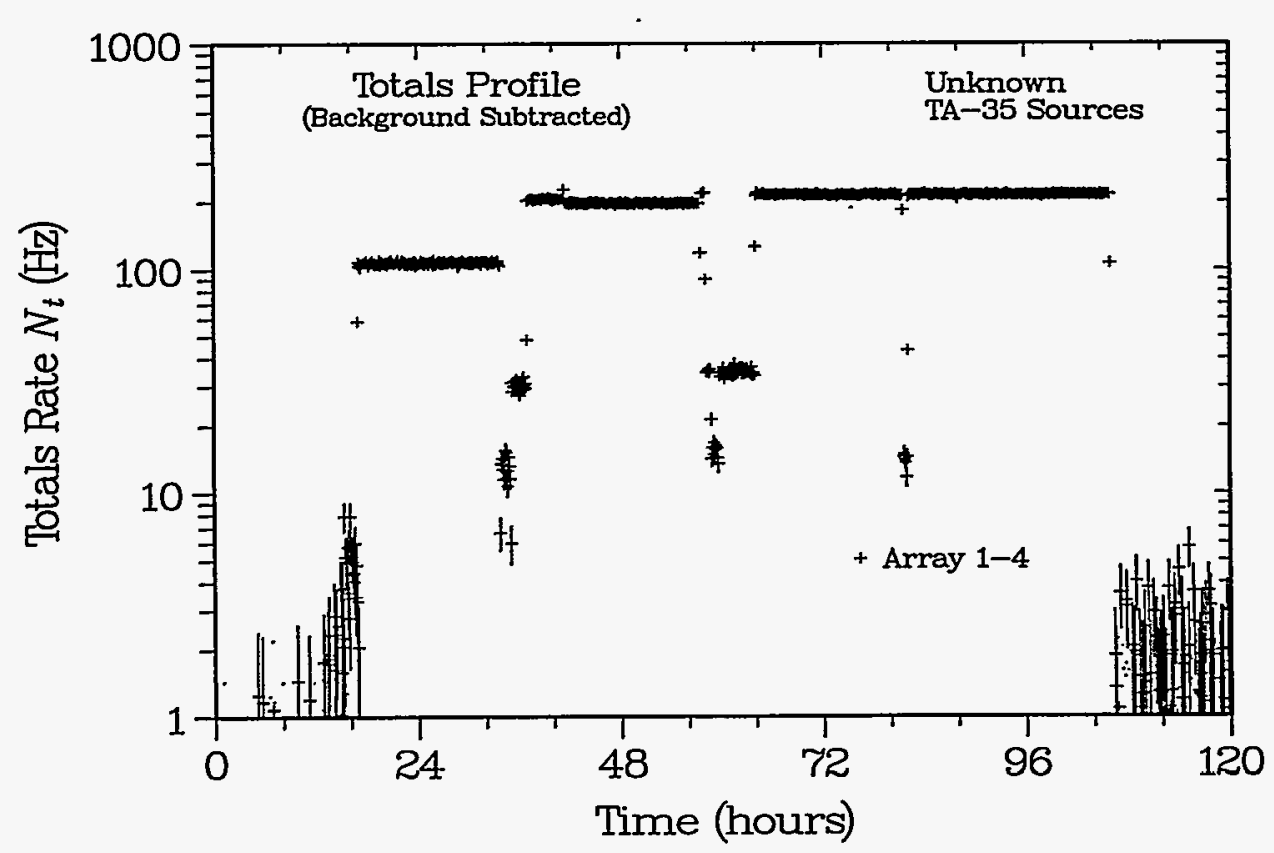

Fig. 6.6a. Total count rate for array 1-4 as a function of time obtained while a series of tests using unknown sources were being made in the same room with the multielement arrays. Four different accumulation intervals are visible.

neutrons from the Am- $\mathrm{Li}$ source. Thus, nonzero asymmetries provide a definite indicator for a nearby radiation source, because natural backgrounds give near-zero asymmetries. The corresponding angle values reported by array 1-4 are shown in Fig. 6.6c, with the solid line 


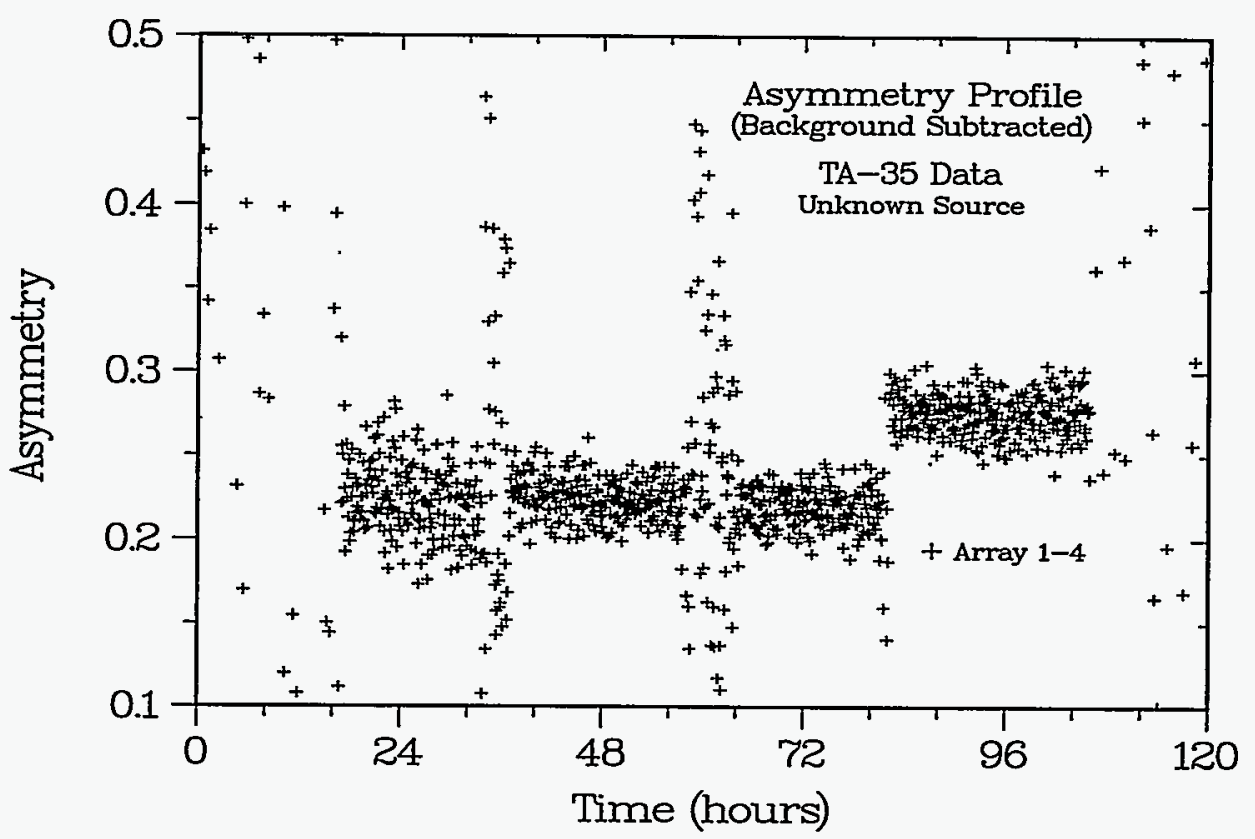

Fig. 6.6b. Time-dependent asymmetry values observed with an unknown radiation source located $4.7 \mathrm{~m}$ away.

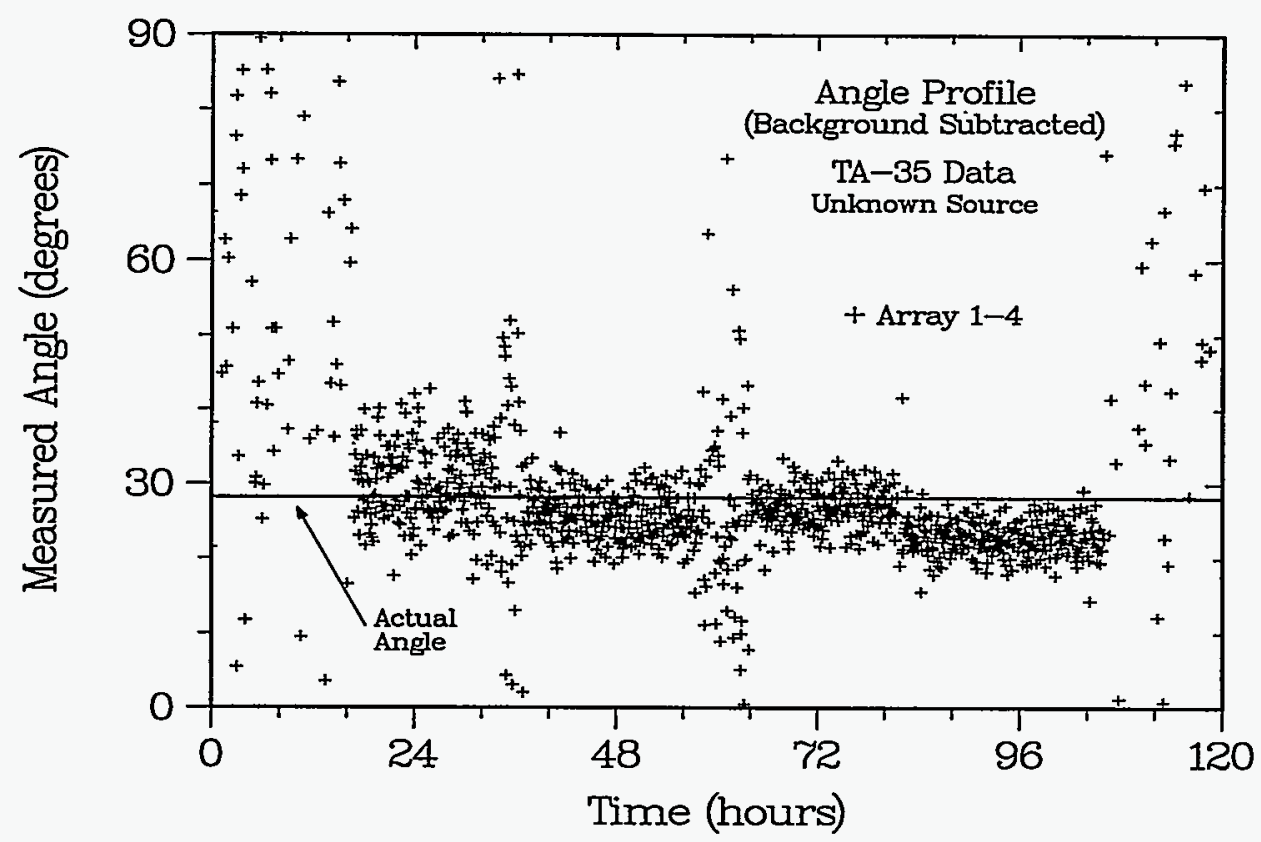

Fig. 6.6c. Angle measurements made by using array 1-4 to track an unknown radiation source.

at $28.2^{\circ}$ indicating the expected result. As in the count-rate and asymmetry measurements, the behavior of the angle values over the periods between the major measurement intervals indicates that smaller calibration sources were being placed in the counter. The slight changes in the angle values for different segments may be related to changes in the amounts of direct and scattered fluence as the source energies are changed. 
TA-35 Summary. With the improvements in counting statistics and detector geometry provided by the experiments in this chapter, it now appears that the detector's fundamental angle accuracy is consistent with the $\pm 10^{\circ}$ values obtained in the original computer simulations in Ref. 1. For the more practical examples in this chapter, however, the actual angle accuracies are more affected by room scattering, as shown clearly by the measurements from Secs. 6.1 and 6.2 with the source or detector placed next to a shielding wall. With this important qualification, the rangefinder technique developed in the last two chapters appears to be basically sound, but its utility must be carefully evaluated for the application at hand.

\section{SUMMARY AND CONCLUSIONS}

Experimental Arrangement. Chapter 2 presents a complete description of the detectors, sources, and experimental layout. The focus is on nondirectional measurements, particularly the dependence of count rate on source separation, as used in conventional grid-type searches. The results emphasize the importance of counting statistics and background corrections and the utility of the approximately $1 / r^{n}$ count-rate dependence.

Basic Directionality. Even the simplest results in Chap. 3 clearly demonstrate that segmenting a detector can provide valuable directional information, at the least an association between maximum count rates and source quadrants. More complex is active collimation, which uses software to restrict the detector response to cover only a certain angular range, typically about $180^{\circ}$. Perhaps the major result of this chapter, however, is the good agreement between the various measurements and the original computer simulations of basic directionality.

Advanced Techniques. Instead of electronically producing a detector that is sensitive only in certain directions, Chap. 4 reverses the approach to develop an omnidirectional detector that can detect an unknown radiation source and estimate its direction. In particular, the asymmetry approach uses detector analyzing powers and source polarizations to provide a convenient framework for interpreting the measurements, which forms the basis for the analyses employed in the remaining chapters.

Rangefinder Results. The CMR and TA-35 experiments in Chaps. 5 and 6 begin by using individual-rod count rates to demonstrate the importance of correlations between multiple detectors. Given the limitations of a direction-only analysis for moving sources, a pair of directional detectors can be used to develop a rangefinder for radiation sources. First, the intersections between the direction vectors from each array are used to provide a set of absolute source locations. Second, because these intersections do not occur in every case and are not uniformly distributed, they are used to calibrate the general $k / r^{n}$ relation between count rate and source distance. This calibrated relation is used to provide a complete set of source distances, which are finally combined with the corresponding direction vectors to give a complete set of source locations. This approach is used for both the moving sources in the CMR and TA-35 facilities and for the high-statistics, stationary-source measurements made at TA-35.

Room Scattering. With good counting statistics and a longer base line between the two arrays, the limit on location accuracies becomes the ratio between the direct and scattered fluences observed at the detector location. If the detector or source is placed next to a massive wall, therefore, the reported direction vector may well point toward the wall instead of the source. This behavior becomes particularly likely at large source distances, but its ultimate importance depends on the details of the intended application. 
Source Searches. The original application for the directional techniques in this study was expected to be searches for nuclear materials. As described in some detail in Ref. 2, a conventional search technique attempts to determine the source location from differences between count rates measured at different points across a grid. In this approach, changes in background levels are usually the limiting factor. The present approach is based on the fact that a radiation gradient is itself unexpected, so the searcher simply moves in the direction indicated by the detector. In the ideal case, the studies in Ref. 2 suggest that directional searches should have roughly a twofold advantage over a conventional search using a detector of the same size. As we have seen in Chap. 6, however, more important restrictions may be imposed by the combination of room scattering and counting statistics.

Vault Surveillance. The second application under consideration uses one or more directional detectors to monitor the radiation field in a storage vault. Because each detector indicates only the average field direction, individual sources cannot be recognized. However, a change in the position or output for any of the sources will disrupt the average field, as may the intrusion of any attenuating object, such as a person. By calculating differences from a long-term average or between sequential accumulations, it should be possible to provide a time-dependent analysis of such changes. The present studies indicate that this technique is promising, but it will certainly require additional study. In particular, moving a single source produces only a small change in the net field in a vault containing a large number of sources, so long accumulations and excellent detector stability will be needed.

Further Work. Our previous studies were mainly calculational, with little data available to test the validity of assumptions. Partly because of the complex nature of the present detectors, this report relies heavily on empirical data, not on simulations. Although the exercises with moving or unknown sources in the last chapter are fairly realistic, additional studies will be needed to refine the techniques or to optimize a detector for a particular application.

\section{ACKNOWLEDGMENTS}

This study is a follow-on to previous work with other collaborators as described in the references. The present detector hardware and NCC data-acquisition system were developed in association with Mike Miller of the Safeguards Science and Technology Group (NIS5) for use in studies of advanced multiplicity counting and transparency techniques. The current adaptation of the equipment was encouraged by Bob Scarlett of LANL's Advanced Nuclear Technology Group (NIS-6), and it was partially funded by the Office of Research and Development of the U.S. Department of Energy's Office of Nonproliferation and National Security. Parrish Staples of NIS-5 provided many valuable comments and a final review of the completed manuscript. 


\section{REFERENCES}

1. R. C. Byrd, "Directional Neutron Detectors (U)," Los Alamos National Laboratory report LA-12379-MS (U) (October 1992).

2. Roger Byrd, George Auchampaugh, and William Feldman, "Directional Measurements for Sources of Fission Neutrons (U)," Los Alamos National Laboratory report LA-12633MS (U) (November 1993).

3. R. C. Byrd, G. P. Estes, and C. R. Mannon, "Far-Field Fast-Neutron Energy Spectra from an Unshielded Fission Reactor (U)," Los Alamos National Laboratory report LA12870-MS (U) (March 1995).

4. R. C. Byrd, J. D. Drabanski, and B. L. Barraclough, "Monte Carlo Calculations of the Effective Area and Directional Response of a Polyethylene-Moderated Neutron Counter (U)," Los Alamos National Laboratory report LA-12850-MS (U) (January 1995).

5. M. C. Miller, "Fingerprinting Nuclear Weapon Components (U)," Defense Science (Summer 1996), Los Alamos National Laboratory document LACP-96-37 (SRD) (May 10, 1996), pp. 84-87.

6. D. G. Langner, M. S. Krick, and K. E. Kroncke, "A Large Multiplicity Counter for the Measurement of Bulk Plutonium (U)," Nuclear Materials Management XXIII (1994 Proceedings Issue) (U), 474-479 (1994).

7. J. K. Halbig et al., "Portable Shift Register (U)," Nuclear Materials Management XXIII (1994 Proceedings Issue) (U), 487-493 (1994).

8. M. S. Krick and W. Harker, "Multiplicity Neutron Coincidence Counting User Manual (U)," Los Alamos National Laboratory document LA-UR-93-1394 (U) (1993). 